University of Louisville

ThinkIR: The University of Louisville's Institutional Repository

Electronic Theses and Dissertations

$5-2020$

\title{
Development of rationally designed live attenuated vaccines for Lassa fever and Venezuelan equine encephalitis.
}

Dylan MacGregor Johnson

University of Louisville

Follow this and additional works at: https://ir.library.louisville.edu/etd

Part of the Virus Diseases Commons

\section{Recommended Citation}

Johnson, Dylan MacGregor, "Development of rationally designed live attenuated vaccines for Lassa fever and Venezuelan equine encephalitis." (2020). Electronic Theses and Dissertations. Paper 3393.

https://doi.org/10.18297/etd/3393

This Doctoral Dissertation is brought to you for free and open access by ThinkIR: The University of Louisville's Institutional Repository. It has been accepted for inclusion in Electronic Theses and Dissertations by an authorized administrator of ThinkIR: The University of Louisville's Institutional Repository. This title appears here courtesy of the author, who has retained all other copyrights. For more information, please contact thinkir@louisville.edu. 


\title{
DEVELOPMENT OF RATIONALLY DESIGNED LIVE ATTENUATED VACCINES FOR LASSA FEVER AND VENEZUELAN EQUINE ENCEPHALITIS
}

\author{
By \\ Dylan MacGregor Johnson \\ B.S., University of Hawaii 2008 \\ M.S., Indiana State University 2014 \\ M.S., University of Louisville, 2018
}

\begin{abstract}
A Dissertation
Submitted to the Faculty of the School of Medicine of the University of Louisville in Partial Fulfillment of the Requirements for the Degree of

Doctor of Philosophy in Microbiology and Immunology
\end{abstract}

Department of Microbiology and Immunology University of Louisville Louisville, Kentucky

May 2020 

DEVELOPMENT OF RATIONALLY DESIGNED LIVE ATTENUATED VACCINES FOR LASSA FEVER AND VENEZUELAN EQUINE ENCEPHALITIS

\author{
By \\ Dylan MacGregor Johnson \\ B.S., University of Hawaii 2008 \\ M.S., Indiana State University 2014 \\ M.S., University of Louisville 2018
}

A Dissertation Approved on

March 3, 2020

By the following Dissertation Committee:

Igor S. Lukashevich, MD,PhD, DSc

Thomas C. Mitchell, PhD

Donghoon Chung, PhD

Jill M. Steinbach-Rankins, PhD

Kenneth E. Palmer, PhD 


\section{DEDICATION}

I dedicate this dissertation to the memory of my grandmothers,

Sylvia "Grams" Coleman

and

Margaret F Johnson

who always provided unconditional love and support, and to the memory of

Alex Johnson

who always reminded me to enjoy life. 


\section{ACKNOWLEDGEMENTS}

I would like to express my deepest appreciation for the guidance and mentorship of Dr. Igor S Lukashevich. I would also like to thank my committee members, Dr. Donghoon Chung, Dr. Kevin Sokoloski, Dr. Thomas Mitchell, Dr. Jill Steinbach-Rankins, and Dr. Kenneth Palmer for their input and support. This work would not have been possible without the assistance of my collaborators, Dr. Juan Carlos de la Torre, Dr. Peter Pushko, Dr. Slobodan Paessler, and Dr. Irina Tretyakova. I am very grateful to Jenny D. Jokinen and Beatrice Cubitt for the excellent laboratory training they provided. I am also thankful for the research support I received from the of the University of Louisville Center for Predictive Medicine, particularly Dr. William Severson, Dr. Jon Gabbard, Marlene Steffen, Dr. Torsten Hopp, Dr. Dan Cramer, Tia Pfeffer, Clarissa Cowen, Dr. Yong-Kyu Chu, Dr. Julie Stotsky, and Robert Scott Adcock; from the University of Louisville Research Resource Facilities, especially Dr. Karen Powell and Jennifer Kraenzel; and from the faculty of the University of Louisville Department of Microbiology and Immunology, especially Dr. Pascale Alard, Dr. Michelle Kosiewicz, Dr. Matthew Lawrenz, Dr. Russell Salter, Dr. Haval Shirwan, and Dr. Esma Shirwan Yolcu. I am also thankful for the training that provided a wonderful foundation from Dr. Swapan K Ghosh, Dr. Joy O’Keefe, Dr. Kand McQueen, Dr. Richard Fitch, Dr. William L Gosnell, Dr. Sandra Chang, Dr. Abby Collier, Dr. Alan Katz, Dr. Paul Patek, Mike Remer, Glen Cook, Mike McPheeters, Frank Holley, Dan Shortenhaus, Barbra Denny, and Patrick Russi.

I would also like to express my thanks to my loving wife, Emily, for being my cornerstone. Also, my gratitude to my family: Mary Ellen, Russ and Mark Thurow; Carrie, John and Jacob Gillette; and Carl, Connie, Maggie, and Preston Johnson, and Melvin Roth. 


\section{ABSTRACT \\ DEVELOPMENT OF RATIONALLY DESIGNED LIVE ATTENUATED VACCINES FOR LASSA FEVER AND VENEZUELAN EQUINE ENCEPHALITIS \\ Dylan M Johnson \\ May 9, 2020}

Lassa Virus (LASV) and Venezuelan Equine Encephalitis Virus (VEEV) are two single stranded RNA viruses belonging to the Arenavirus and Alphavirus families, respectively. Both are emerging pathogens without approved vaccines or treatments. VEEV is an important biothreat pathogen because it has been weaponized and is extremely infectious as an aerosol. VEEV causes biphasic febrile illness that can progress to a viral encephalitis with low mortality, but high morbidity. LASV causes a viral hemorrhagic fever, Lassa Fever (LF), which is endemic in West Africa, and responsible for between 100-500k annual infections with a 1-2\% overall mortality rate. ML29 and VEEV TC-83 are well-described live attenuated vaccines based on LASV and VEEV, respectively. This work describes strategies to further attenuate and enhance the safety of ML29 and TC-83, an important step in preclinical development.

VEEV V4020 was designed based on the stabilization of an E2 mutation, and rearrangement of the structural genes of TC-83. Here, VEEV V4020 is shown to be more attenuated than TC-83 based on IC inoculation in mice, and more phenotypically stable, in terms of pathogenicity, during serial passaging.

ML29 is a reassortant virus combining the immune dominant glycoprotein (GPC) and nucleoprotein (NP) of LASV with the replicative machinery of to Mopeia virus (MOPV), a nonpathogenic relative of LASV. We provide evidence ML29 is safer and more immunogenic 
than MOPV in STAT-1 deficient (STAT- ${ }^{-/}$) mice and Hartley guinea pigs. Additionally, Defective Interfering Particles (DIPs) from ML29 enhance vaccine immunity and attenuation in STAT-1 ${ }^{-}$ mice, and in intracranial inoculations of $\mathrm{CBA} / \mathrm{J}$ mice.

A potential mechanism of attenuation for ML29 is presented based on small RNAs detected from the ML29 L-segment by Northern Blot (NB). Furthermore, a unique RNA band was associated with DIP enriched ML29 was detected. A L-segment based Minigenome System (MG) for arenaviruses is described, which can be used for future analysis of the mechanism of replication for reassortant arenaviruses.

The advanced safety data for both ML29 and VEEV V4020, described in this dissertation, combined with non-human primate efficacy studies described elsewhere, supports the advancement of both of these experimental vaccines to human clinical trials. 
DEDICATION ...iii

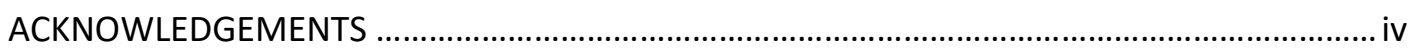

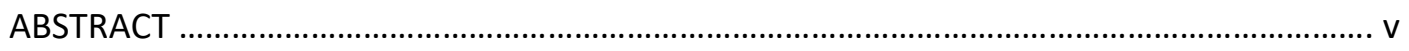

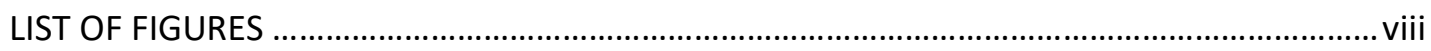

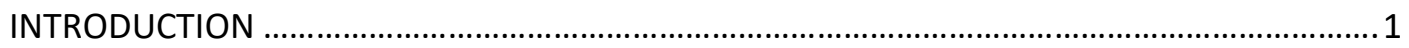

NEW CHALLENGES FOR DEVELOPMENT OF A LASSA FEVER VACCINE WITH FULL

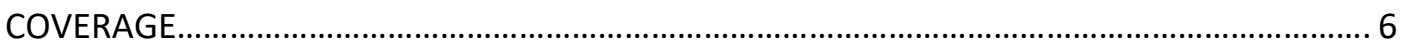

ATTENUATED REPLICATION OF LASSA VIRUS VACCINE CANDIDATE ML29 IN

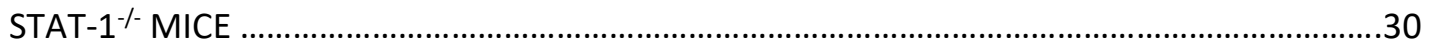

SAFETY AND IMMUNOGENICITY OF A DEFECTIVE INTERFERING PARTICLE ENHANCED LASSA VIRUS VACCINE CANDIDATE IN STAT-1 ${ }^{-/}$MICE, CBA MICE, AND GUINEA PIGS .......... 55

ADVANCED SAFETY AND GENETIC STABILITY IN MICE OF A NOVEL DNA-LAUNCHED VENEZUELAN EQUINE ENCEPHALITIS VIRUS VACCINE WITH REARRANGED STRUCTURAL GENES

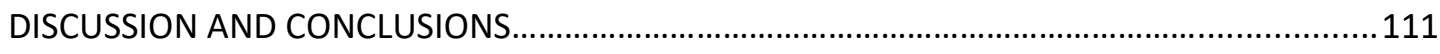

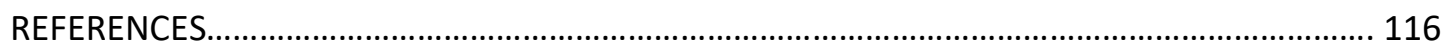

CURRICULUM VITA 


\section{LIST OF FIGURES}

FIGURE

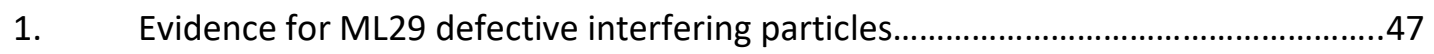

2. ML29 and ML29P50 attenuation in STAT-1-/- mice.......................................... 48

3. Histology showing attenuated ML29 and ML29P50 in STAT-1-/- ........................ 50

4. Immunogenicity of ML29, ML29P50, and MOPV in STAT-1-/- ............................... 51

5. Cytokine responses to ML29, ML29P50, and MOPV in STAT-1-/- mice.....................51

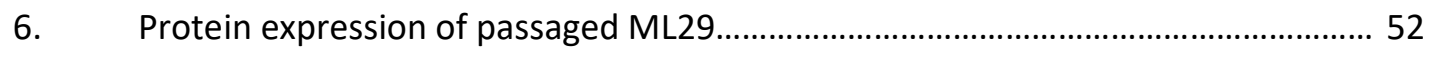

7. ML29 and ML29P50 attenuation in $129 S 6$ mice................................................. 53

8. Cytokine responses to ML29, ML29P50, and MOPV in 192S6 mice.......................... 53

9. Passaged ML29 interferes with LCMV-Arm-GFP/GFP reporter expression.............. 75

10. Northern blots of ML29, ML29P28, ML29P53, LASV, and MOPV ............................. 75

11. L-Segment LCMV minigenome system............................................................. 76

12. Attenuation of ML29 and ML29P50 in STAT-1-/- and CBA/J mice............................77

13. Immunogenicity and safety of ML29 and ML29P50 in Hartley guinea pigs............. 78

14. VEEV V4020 and VEEV TC-83 design and titers after mouse IC inoculation.............. 100

15. Histological kinetics of VEEV V4020 compared to TC-83 brain infection................... 102

16. NanoString immunogenicity of VEEV V4020 compared to VEEV TC-83 .................. 103

17. Passaging of attenuated VEEV in mouse brain......................................................... 105

18. Next Generation Sequencing genetic variation of passaged attenuated VEEV....... 106

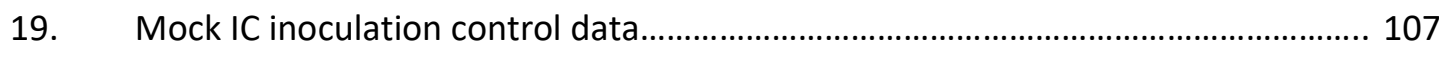

20. Attenuated VEEV NanoString KEGG pathway analysis............................................. 108

21. Additional NGS genetic variation analysis of passaged attenuated VEEV................ 109

22. Mouse Host Transcriptome analysis....................................................................... 110 


\section{CHAPTER I}

\section{INTRODUCTION}

Live Attenuated Vaccines (LAV) are responsible for many of the most successful vaccination campaigns, including the eradication of small pox (caused by Variola virus) and Rinderpest, and the near eradication of Polio [1]. LAVs have also proven useful for many other viral diseases including Influenza [2], Varicella Zoster Virus [3], Measles virus [4], Rotavirus [5], Ebola virus [6], and Yellow Fever virus [7], among others.

LAVs are predominantly based on attenuation of the disease causing strain, however notable exceptions exist including the Vaccinia virus vectored small pox vaccine, and the vesicular stomatitis virus or adenovirus vectored Ebola virus vaccines $[1,6,8]$. While vector based vaccines have the advantage of increased versatility, they often engage the host immune system in different ways than the natural infection [9-11]. This can affect the magnitude and duration of host responses, along with the potential for the generation of vector focused immune responses [9-11]. Because of this, vaccines based on the naturally occurring pathogen are often preferred. However, because LAVs are often derived from pathogenic viral strains, concerns remain about the possibility of reversions to pathogenic phenotypes arising $[12,13]$. This dissertation focuses on the development of LAVs that target two emerging single-stranded RNA viruses, Lassa virus (LASV) and Venezuelan Equine Encephalitis Virus (VEEV). Both of the LAVs are based on attenuation of their respective naturally occurring viruses.

ML29 Live attenuated vaccine for LASV 
Mopeia virus (MOPV), a virus closely related to LASV which does not cause pathogenesis in humans or non-human primates, has documented immunogenicity that spans across LASV genetic clades $[14,15]$. However, MOPV is widely considered insufficiently attenuated for use as a LAV [15]. ML29, a genetic reassortant between MOPV and LASV (with antigenic determinants of LASV and the replicative machinery of MOPV,) is a leading LASV vaccine candidate that protects non-human primates (NHP) from fatal challenge [16-18]. Current approaches for LASV vaccine design are forgone in this introduction in favor of extensive discussion in Chapter II. In this dissertation, we test the hypothesis that ML29 is attenuated compared to MOPV, and examine the potential for the inclusion of defective interfering particles to further attenuate ML29 while enhancing the broadly protective immunogenicity of the LAV.

\section{Challenges for the development of safe and effective VEEV vaccines}

VEEV is maintained in avian and rodent species in South and Central America. It is a vector-borne virus, which is transmitted primarily by Culex mosquitos. When the mosquito subsequently transmits the virus to an equine while taking a blood meal, VEEV replicates to high levels, leading to fever, leukopenia, neurological disease, and up to a $83 \%$ mortality rate [19, 20]. During the illness, high levels of viremia allow an infected equine to transmit the virus back to the mosquito vector allowing for subsequent rounds of infection, along with spillover into humans. Humans infected with VEEV typically experience a biphasic febrile illness, and malaise, which can be accompanied by severe headaches. In $4-14 \%$ of individuals, VEE will progress to a viral encephalitis resulting in clinical neurological symptoms, although VEEV has a low case fatality rate humans [19]. VEEV is grouped into six subtypes; however, epidemics have only been associated with subtype I, variants $A B$ and $C$ [21]. 
Early research on VEEV resulted in a number of documented laboratory acquired infections, despite measures to prevent aerosol transmission [22]. The high rate of human infection following aerosol exposure, accompanied by the incapacitating nature of the illness lead to weaponization efforts by both the United States (US) and former Soviet Union [23]. As a result, in the US, VEEV is currently regulated under the Federal Select Agent Program as a biological agent that has the potential to pose a severe threat to both public health and safety and animal health and safety.

The first VEEV vaccine efforts centered on formulations of formalin inactivated circulating VEEV IAB strains. Incomplete inactivation of these viruses led to vaccine-associated epidemics of VEEV IAB $[19,24,25]$. While the reservoir for VEEV IAB remains cryptic, VEEV IC strains periodically emerge through mutation in the ID strains [26]. The attenuated vaccine strain VEEV TC-83 was developed by passaging the virus in guinea pig hearts cell in culture while monitoring for virulence [13]. Although the introduction of VEEV TC-83 drastically reduced laboratory acquired infections [22], it has been stalled in Phase II clinical trials due to incomplete protection and the prevalence of adverse drug effects (ADE) that mirror some clinical features of wild-type disease [27-29]. Despite the high rate of ADE, including fever and headache [30], VEEV TC-83 is still used as an investigational new drug (IND) to vaccinate at risk laboratory workers and soldiers.

Another negative aspect of VEEV TC-83 is that it often fails to induce protective immunity. A formalin inactivated form of VEEV TC-83, C-84, has been investigated for use as a standalone vaccine and to boost the immunity of non-responders to TC-83 vaccination [31]. Unfortunately, C-84 is poorly immunogenic and requires frequent re-vaccination [30, 32]. 
VEEV V3526, a rationally designed vaccine based on a lethal mutation in the PE2 glycoprotein cleavage site, along with a suppressor mutation in the E1 glycoprotein at position 253 , is immunogenic and protective. Yet VEEV V3526 remains deeply attenuated in mice, equines and non-human primates [33-36]. Additionally, VEEV V3526 appears to result in less adverse drug reactions in mice and non-human primates than VEEV TC-83 [35, 36]. Despite great promise, the VEEV V3526 failed in phase I clinical trials due to unacceptable reports of moderate to severe fever following vaccination [32].

Recently, chimeric alphaviruses have been considered as LAVs to protect against VEEV. A chimera generated from the structural proteins from VEE combined with the replicative machinery of a related alphavirus with low pathogenicity in humans, Sindbis Virus, was attenuated in mice while providing protecting from lethal VEEV challenge [21, 37]. A similar construct for Eastern Equine Encephalitis protected non-human primates (NHP) from a lethal aerosol challenge [38]. Eilat Virus (EILV) is an alphavirus that only replicates in mosquitos, and is unable to infect mammalian or avian cells in culture due to host restriction for both entry and RNA replication $[39,40]$. Chimeric viruses with EILV replicative machinery and VEE structural proteins were safe in mice, and protected them from lethal VEEV challenge [41]. However, in this case, the chimeric virus is functioning more as a replicon than a LAV, as it likely does not replicate following vaccination. Additionally, EILV can interfere with VEEV replication potentially extending the window for use of this chimeric vaccine in post-exposure prophylaxis $[41,42]$.

VEEV V4020 is a vaccine candidate based on VEEV TC-83 with rearrangement of the structural genes, a stabilizing mutation in the key E2-120 glycoprotein attenuating mutation, and the addition of a second subgenomic 265 promoter, leading to glycoprotein and capsid genes being produced independently $[43,44]$. The additional promoter likely changes the ratio of glycoprotein to capsid production in vivo, which could interfere with viral particle assembly. 
Previously, we showed that VEEV V4020 provided complete protection with sterilizing immunity following lethal challenge of VEEV-TrD in mice [44]. Here, we test the hypothesis that VEEV V4020 is attenuated compared to VEEV TC-83 in terms of neuro-virulence in mice, and that it has reduced capacity for reversion to a pathogenic phenotype compared to TC-83.

In this work, we provide evidence to support the overarching hypothesis that LAVs for LASV and VEEV, based on their respective natural pathogens, are safe and immunogenic. Additionally, two methods to enhance the immunogenicity and safety of the vaccine candidates are tested: the inclusion of defective interfering particles for the enhancement of the ML29 vaccine, and the rearrangement of structural genes for enhancement of the Venezuelan Equine Encephalitis Virus (VEEV) TC-83 vaccine. Advanced small animal models are used to compare the safety and immunogenicity of ML29, defective interfering particle enhanced ML29, and VEEV TC-83, and VEEV V4020. 
CHAPTER II

NEW CHALLENGES FOR DEVELOPMENT OF A LASSA FEVER VACCINE WITH FULL COVERAGE

Lassa virus (LASV) is the most prevalent African arenavirus, existing in nature as a persistent infection of rodent hosts. In particular, Mastomys natalensis, which are widely spread in sub-Sahara Africa. While LASV does not affect its natural host, rodent-to-human transmission can result in Lassa Fever (LF) disease with high mortality rates in vulnerable groups. Recent improvement in virus detection and rodent host identification provided evidence of LASV expansion outside of traditional endemic areas including Nigeria, Guinea, Liberia and Sierra Leone. Recently, Nigeria experienced the largest ever LF outbreak with an unusually high case fatality rate of $27 \%$ in 2018 and $20.4 \%$ in 2019. The World Health Organization (WHO) included LASV in top priority pathogens, and released a Target Product Profile (TPP) for LASV vaccine, which raised challenges for vaccine developers to produce a vaccine with pan-LASV coverage. Rationally designed and platform-based vaccine candidates will be overviewed, and how they will meet the WHO's TPP criteria for development of a prophylactic LASV vaccine with full coverage will discussed.

\section{Introduction}

Lassa virus (LASV) is a rodent-borne human pathogen from the Arenaviridae family.

Currently, the family includes four genera, Antennavirus, Hartmanivirus, Mammarenavirus, and Reptarenavirus [45-47]. These viruses infect fish (antennaviruses), snakes (hartmaniviruses and reptarenaviruses) and rodents (mammarenaviruses). LASV belongs to the Old World group (OW 
or Lymphocytic Choriomeningitis Virus, LCMV-LASV serocomplex) of the genus

\section{Mammarenavirus.}

Mammalian arenaviruses have 2 genomic single-stranded ambisense RNA segments.

The L RNA encodes large L (RNA-dependent RNA polymerase) and small RING finger Z (matrix) proteins. The S RNA encodes a nucleoprotein (NP) that tightly associates with viral RNA, and a glycoprotein precursor (GPC), which is processed into three subunits: the stable signal peptide, SSP; the receptor-binding GP1; and the transmembrane GP2, which is responsible for fusion with cell membrane. All three subunits are non-covalently linked to form trimeric features on the virion surface [48]. Surface GPs are extensively glycosylated and complex pattern of this glycosylation complicates development of GPC-based vaccines [45, 49-51]

LASV originated in Nigeria about $800-1000$ years ago and gradually moved westward during the last few centuries [52-54]. LASV viral polymerase was identified as a major positive selection target during this migration [55]. The LASV species represent a collection of genetically heterogeneous isolates from rodents and humans, resulting in their placement into 6 genetic lineages based on geographic location. The most heterologous LASV strains circulates in Nigeria (lineages I-III) and Guinea, Liberia and Sierra Leone (lineage IV) with sequence inter-lineage diversity up to $24.6 \%[45,53,54,56]$. A fifth lineage has been proposed for LASV isolates from Mali and Ivory Coast $[54,57]$. An isolate from a cluster of human infections epidemiologically linked to Togo was proposed to represent a lineage VI [58].

Persistently infected multimammate rats (Mastomys natalensis spp.), a widely spread and genetically diverse rodent species in Sub-Sahara Africa, are the major source of LASV infection which can vary from asymptomatic or flu-like disease to fatal Lassa fever (LF) responsible for thousands of death annually. With the exception of dengue fever, the estimated 
global burden of LF is the highest among the viral hemorrhagic fevers [59]. In contrast to poorly predicted EBOV outbreaks, LASV infection is endemic in West African countries, representing $5 \%$ of the total world population, including the most populated Nigerian state $[60,61]$. New epidemiological studies indicate that LASV endemic regions are expanding [62]. One environmental-mechanistic study predicts that the number of LASV infections will double from the current levels in coming years [63]. Taking into consideration the impact of LASV infection on public health in endemic areas, the WHO included LASV in its top-priority pathogens list, and released a Target Product Profile (TPP) for LASV vaccine development [64]. In 2018, the US Food and Drug Administration (FDA) added LASV to their priority review voucher program.

The WHO'S TPP is focused on a preventive vaccine for the safe protection of healthy adults and children, with a duration of at least 3 years, against LASV strains from the major genetic lineages, I-IV. The WHO's prequalification requirement included in this TPP indicated the preferred vaccine candidate must be inexpensive, safe, highly efficacious, heat-stable, multiantigen, and user- and environmentally friendly. Here, we will review the current status of LASV vaccine research focusing on several challenging areas of pre-clinical and clinical development.

LASV genetic diversity likely contributes to the underestimating of its prevalence in West Africa $[65,66]$ and seems to be also associated with wide range of clinical manifestations and outcomes in different endemic areas. LASV diversity represents a huge challenge for diagnostics and vaccine development [67]. Currently, there is no licensed vaccine against LF and therapeutic options are limited to off-label use of Ribavirin.

\section{Rodent-to-human transmission}

Although M. natalensis species are widely spread in sub-Sahara Africa, LASV infection is predominantly confined to M. natalensis monophylogenic group A-I in West Africa [68]. Other 
host phylogroups from Central and Southern African regions have been associated with nonpathogenic Mopeia (MOPV), Morogoro (MORV), Gairo (GAIV) and Luna (LUNV) mammalian arenaviruses [69-72]. In these areas, LASV has not been found, and LF cases have never been reported. MOPV has been considered as a "natural LASV vaccine" since experimentally immunized non-human primates (NHPs) were fully protected against fatal LF $[15,73,74]$; and thus, the MOPV genetic backbone was used to rationally design promising LASV vaccines [17, 75].

Natural rodent-to-human transmission of LASV occurs primarily via inhalation or/and ingestion of dusts contaminated with excreta of infected rodents [76-79]. The peri-domestic life style of M. natalensis, house design, and poor sanitation in rural areas provides an ideal environment for rodent activities at night, and even during the day [80, 81]. Rodent excreta in food stocks and consuming rodents meat have been identified as major risk factors of LASV transmission [77]. Clusters of infected $M$. natalensis are responsible for the spatial distribution of LF cases in endemic areas [63, 82-84]. In LASV endemic areas, contaminated rodent excreta is a source for the natural infection of NHPs as recently documented in Southern Nigeria [85].

\section{Mucosal routes of LASV infection}

Mammarenaviral infection is dependent on directional exposure to, and release from, polarized epithelial cells. The pattern of LASV interaction with its target cells during entry can drive the pathogenicity observed during natural infection $[86,87]$. In polarized renal epithelial

MDCK cells, LASV entry occurred more efficiently from basolateral surfaces, while matured virus particles were predominantly released apically [88]. A basolateral route of entry suggests that the virus can only access cell receptors when the integrity of the epithelial barrier has been compromised [89]. The relation of the in vitro LASV - receptor interaction pattern to renal 
dysfunction in LF patients in Nigeria, [90] and virus shedding in urine [81], remains to be elucidated.

The microbial community associated with the gastro-intestinal mucosa may affect susceptibility to LASV infection. Pre-exposure of epithelial cells to Salmonella enterica promoted the entry of lentivirus pseudo particles expressing LASV GPs [91]. This finding suggests that the high burden of Salmonella infections in Africa can be an additional risk factor for individuals living in LASV endemic areas [92].

Polarized epithelial cells serve as a barrier restricting virus infection [93]. Accumulating evidence indicates that crossing a healthy epithelia barrier at site of the infection can contribute to virus attenuation. In a surrogate model of LF in NHPs, LCMV-WE54 applied systemically (intravenously) always resulted in fatal LF-like disease. However, oral application (lavage) resulted in limited infection, and the development of protective immunity [94-96]. LASV infectivity was only marginally decreased at $\mathrm{pH} 4.0$ [97], which suggests that the low pH gastric environment does not substantially affect LASV infectivity. If so, this observation indicates rodent-to-human transmission via the gastro-intestinal tract as one of the major routes of natural infection [76, 77].

Gastric infection may confer natural immunity to LASV. Experimentally, mice orally immunized with recombinant S. typhimurium expressing LASV NP were protected against lethal LASV challenge [98]. Similarly, hamsters experimental inoculated intragastrically with Puumala virus (PUUV), a rodent-borne hantavirus causing hemorrhagic fever with renal syndrome (HFRS), developed protective immunity against lethal PUUV challenge [99]. These observations suggest that the high resistance of LASV to low $\mathrm{pH}$ environments may be an adaptation that facilitates 
transmission via alimentary tract because it results in benign infection and protection from fatal disease.

Human-to-human transmission is estimated to be responsible for about $20 \%$ of LASV infections. It is the major mechanism of transmission during nosocomial outbreaks, and is often associated with a few infected individuals, referred to as "super-spreaders" [100, 101]. The mechanism of super spreading is not clear and remains to be elucidated. In addition, persistence of the virus in urine, combined with poor sanitation practices, can facilitate LASV transmission during nosocomial outbreaks.

\section{LASV cell receptor usage diversity}

After crossing an epithelial barrier, LASV targets macrophages and dendritic cells (DCs) and via the blood stream (viremia), leading to infection of many different tissues and organs (pan-tropism). The major cellular receptor for LASV, MOPV, and LCMV and some NW mammalian arenaviruses is $\alpha$-dystroglycan, $\alpha D G$ [102]. The $\alpha D G$ protein is non-covalently linked to a transmembrane subunit, $\beta D G$, which transduces signals from the Extra Cellular Matrix (ECM) to the intracellular cytoskeleton. Like-acetylglucosaminyltransferase mediated $O$ mannosylation is crucial for the $\alpha D G$ interaction with GP1, as well as for the interaction of $\alpha D G$ with its natural ligands [103-105]. The strength of LASV GP1 binding affinity to $\alpha$ DG can be partly correlates with pathogenicity. The affinity of LASV and LCMV-WE54 GP1 to $\alpha$ DG was 2-3 logs higher than those of the non-pathogenic viruses MOPV and LCMV-ARM [106]. Notably, viral GP1 binding results in down-regulation of functional $\alpha$ DG on cell surface, which can affect cell-to-cell interaction (e.g., interaction of DCs with T lymphocytes) [105, 107].

While the $\alpha D G$ is the major cell receptor for LASV, the usage of this receptor is cell- and tissue specific depending on $O$-mannosylation, additional signaling interactions (e.g., cellular 
receptor tyrosine kinases, p21-activating kinase), and proper interaction with DG-associated proteins (e.g., utrophin, $\beta$-dystrobrevin, $\beta 2$-syntropin, sarcoglycans) $[108,109]$. In cells lacking functional $\alpha$ DG (e.g., mature hepatocytes), additional molecules, DCs-specific adhesion molecule (DC-SIGN), liver and lymph node sinusoidal endothelial cell C-type lectin, and Axl and Tyro3, two members of the TAM ( $\underline{T} y r o 3, \underline{A} x l, \underline{M} e r)$ family, promote $\alpha$ DG-independent LASV infection in vitro [110, 111]. In marmosets, the Nigerian LASV/803213 strain induced upregulation of Axl-1 in the liver promoting virus replication and pathological hepatocyte proliferation, contributing to arenavirus-induced liver pathology.

TAM receptors and their ligands (e.g., Gas6, protein S) play an essential role in apoptotic cell clearance, damping immune responses, and in coagulation pathways $[112,113]$. DC-SIGN and LSECtin lectins can also serve as LASV attachment factors on monocyte/macrophages and DCs, early targets of LASV infection [111]. These targets seem to be crucial for the induction of host responses via TLR2/TLR6/CD14 engagement and, Mal/Dy88- and NF-KB-mediated innate immunity pathways [114]. This engagement can contribute to strong innate NF-KB-mediated pro-inflammatory and adaptive immune responses [114, 115]. Despite all this receptor usage complexity, it is becoming clear that receptor engagement induces an array of cell responses that results in internalization of the virus, while also orchestrating of the host cell response.

Upon engagement of the cell receptor, LASV and LCMV enter susceptible cells using an unusual pathway, which is independent of clathrin, caveolin, and actin, factors required for the "classical" endocytic pathway employed by the NW mammalian arenaviruses (e.g., JUNV, MACV). Recent studies demonstrated the involvement of a micropinocytosis-like mechanism in LASV entry $[108,116]$. It was documented that several micropinocytosis-associated kinases, epithelial and hepatocyte growth factor receptors (EGFR and HGFR), and sodium hydrogen 
exchangers (NHE), were required for LASV entry. However, in contrast to "classical" inducible micropinocytosis, LASV entry did not induce morphological changes in host cells.

When virus-containing vesicles are delivered to late endosomes/lysosomes, LASV GP1 is dissociated from $\alpha \mathrm{DG}$ and binds LAMP1 (Lysosome-Associated Membrane Protein 1) to initiate membrane fusion [117]. All LASV strains share the GP1 amino acid residues required for LAMP1 binding, but these residues have not been found in LCMV and MORV. This suggests that among genetically related mammalian arenaviruses, the requirement of LAMP1 is a unique feature of LASV $[117,118]$. LASV GP1 binds N-glycosylated LAMP1 carrying $\alpha-2,3-$ linked sialic acid (SA) in the upper airway of rodents, promoting the horizontal transmission of LASV in the natural reservoir. Meanwhile, $\alpha-2,6$-congugated SA in the human upper airway seems to limit humanto-human transmission. Interestingly, EBOV entry also is critically dependent on the late endosomal/lysosomal protein NPC1 (Niemann-Pick disease type C1 protein), suggesting that this "receptor switch" mechanism can contribute to virus-host interaction and viral tropism in both highly pathogenic infections [119].

The extensive LASV glycan shield on the LASV surface (which comprises 25\% of the glycoprotein mass) efficiently protects vulnerable GP1 sites involved in interaction with aDG, LAMP1, and the GP2 fusion loop from a humoral antibody response $[48,120]$. This shield contains an abundance of unprocessed glycans forming spatially distinct clusters, protecting crucial GP1 and GP2 sites. Evolutionary limited amino acid diversification around these clusters supports their role in immune evasion $[48,53]$. The presence of unprocessed oligomannose-type glycans, and the importance of native quaternary LASV glycoprotein structures, are real challenges for the development of recombinant vaccine candidates based on the expression of LASV GPC, since current expression technologies poorly mimic the native trimeric architecture and glycosylation patterns of mature LASV particles [48]. 


\section{LASV biological diversity and variation in LF clinical manifestations}

LASV is a rodent-borne virus, and LASV infection is handled differently by the immune system of rodents, humans, or NHPs. Not surprisingly, LASV isolates from LF patients with different clinical outcomes showed a weak correlation between the severity of human disease and virulence in experimentally infected guinea pigs $[121,122]$. Most studies in animal models were performed with LASV/Josiah/SL/76/H (LASV/JOS) or related viruses from lineage IV [123]. LASV/JOS and the Nigerian LASV/803213 (lineage II) are highly pathogenic in strain 13 Guinea Pigs (S13GPs) and NHPs, but not in outbred Hartley Guinea Pigs (OHGPs) [122, 124] [125] [126]; while the LASV/GA391 (lineage III) causes fatal infection in OHGPs [127]. The LASV/LP, the prototypic strain from lineage I, induced only mild weight loss in S13GPs [128]. Notably, a LASV isolate from Mali which is estimated to have separated from lineage IV around 200-300 years ago, was only partially lethal in STR13GPs and NHPs [129].

Our knowledge of the natural history of LASV infection in humans is mainly based on limited clinical studies in Sierra Leone and Liberia performed in the late 1970-1980's [130-133]. A prospective case-control study of 441 confirmed LF patients established that the best predictors of disease are a combination of fever, pharyngitis, retrosternal pain and proteinuria $[131,134]$. The best predictors of clinical outcome were fever, sore throat, and vomiting. Meanwhile, a prospective cohort study in Guinea, where LF was infrequently recognized, failed to identify specific predictors of poor clinical outcomes [135]. Progressed LF in children included widespread edema, abdominal distention, and bleeding ("swollen baby syndrome") [136].

During 1969-2016, 33 imported cases of LF were documented in travelers arriving to non-LASVendemic countries from West Africa [137]. Non-African individuals seem to be more sensitive to LASV infection, and most of them developed more distinctive clinical features (fever with 
pharyngitis, sore throat, tonsillitis, conjunctivitis, oropharyngeal ulcers, or proteinuria) [135, 137].

In progressed LF patients, elevated levels of AST in plasma and high viremia were strongly associated with death $[135,138]$. High AST/ALT ratios suggested substantial involvement of non-hepatic tissues in pathogenesis of the disease. In patients with fatal outcomes, updated clinical observations revealed low or undetectable levels of the proinflammatory chemokines, IL-8 and IFN-inducible IP-10 [139]. Elevated levels of TNF- $\alpha$ receptors and IL- 6 were associated with fatal LF. Elevated levels of IL- 6 in plasma is currently being considered as the third biological marker of fatal LF associated with hepatic regeneration [135, 140-142]. Likewise, low levels of IL-6, IL-10, CD40L, AST, ALT, ALP, and BUN in Sierra Leonean LF strongly correlated with survival $[135,143]$.

The first cases of LF in Sierra Leone were described as LASV hepatitis, and limited postmortem histological studies indicated that the liver was one of the most affected organs participating in a systemic breakdown $[140,144]$. With minimal inflammation, hepatocellular necrosis and active hepatocyte proliferation was found in the livers of LF patients. However, the degree of liver damage was not sufficient to implicate hepatic failure as the primary cause of death. The hepatocyte proliferation observed in LF patients from Sierra Leone was also observed in NHPs experimentally infected with LASV/JOS and Nigerian LASV/803213 [126, 142]. However, in spite of strong proliferative signals, LASV did not induce hepatic hyperplasia. In a marmoset model of arenavirus-induced hepatitis, the infection triggered hepatocyte proliferation, which aborted in G1/S transition of the cell cycle $[67,142,145]$. Expression of nonconventional receptors (AxI-1, Tyro3, DC-SIGN, LSECtin), and stimulation of oval cells (hepatocyte progenitors expressing $\alpha D G$ ), promoted virus replication in hepatocytes. This NHP model of arenavirus-induced liver pathology may be helpful to gain insight into mechanisms of 
pathophysiological hepatic proliferation, and can explain the high levels of AST and ALT in LF patients and the contribution of the liver to LF pathogenesis.

A recent retrospective observational study on $291 \mathrm{LF}$ patients treated in the Irrua Specialist Teaching Hospital, Edo State, Nigeria revealed some similarities and differences between LF in Sierra Leone and Nigeria [90]. In both studies, the liver was clearly involved in pathogenesis as lower AST/ALT ratios (of 1.5) among Nigerian patients were more consistent with hepatocyte-affecting arenavirus infection [145]. Likewise, higher viral loads detected by qRT-PCR strongly correlated with poor outcome [146]. However, in contrast to Sierra Leonean LF, sepsis-associated hypothermia rather than fever was a predictor of poor outcome in Nigerian patients. In addition, LF patients under 5 years old or over 55 years old were at greatest risk for death $[90,147]$. Prospective clinical and epidemiological observations provided evidence that Nigerian strains were associated with more severe LF disease than strains from clade IV (Guinea, Liberia, and Sierra Leone). The fatality rate among hospitalized LF patients in Nigeria, $30-50 \%$, was almost 2-fold higher than among patients from other LASV endemic areas [81, 146, 148, 149].

The 2018 LF outbreak in Nigeria was the largest on the record, and affected 20 states across country with an overall case fatality rate of $25.1 \%$, which is unprecedentedly high for natural "rural" LASV infection [150]. The majority of cases (81\%) were from the three southern states, Edo, Ondo, and Ebonyi. Among the confirmed cases, the most common clinical manifestations were fever, headache, vomiting, fatigue, and abdominal pain. While hemorrhage signs were rare (17\%), these signs, as well as unconsciousness and age (41-60 years), were significantly associated with fatal outcome. Higher fatality rates in older Nigerian LF patients were documented in several studies, which was a clear departure from observations in Sierra Leone where mortality among $60+$ year old patients was lower than children $[90,146,150]$. 
The most striking clinical feature of the recent Nigerian LF outbreak was renal involvement, since elevated creatinine and blood urea were associated with fatal outcome [90]. The acute kidney damage in Nigerian patients seemed to be an intrinsic feature of LASV infection, rather than a complication of the disease. Whether the extent of renal involvement is a specific feature of Nigerian LF has yet to be further elucidated. The heterogeneity of LASV strains from clade II which circulate in the Edo state does not exclude the possibility that some of these viruses uniquely cause kidney damage [146].

Non-Nigerian LASV strains also have the potential to induce kidney pathology. LASV/JOS exposure of human blood mononuclear cells affected the expression of genes involved in coagulation pathways including upregulation of HBEGF (heparin-binding epidermal-growthfactor-like growth factor) which is associated with renal failure and glomerulonephritis [78, 151]. Among other genes involved in coagulation, the strong upregulation of THBD (thrombomodulin) could explain why disseminated intravascular coagulation (DIC) is rare in LF patients in comparison with other viral HFs [78].

The 2018 increase in LF cases in Nigeria was not associated with a particular LASV strain. Phylogenetic analysis revealed that LASV genomes were from lineage II (93\% of genomes) and III with relatively high diversity. It has been estimated that both lineages split from a common ancestor more than 200 years ago, and remained stably separated in rodent populations, presumably by natural barriers formed by the Niger and Benue Rivers [152]. Genomic analysis also did not find evidence of a substantial contribution of human-to-human transmission in the recent outbreak which seems to be more related to improvements in diagnostics and public awareness rather that changes in epidemiology of the disease.

\section{Correlates of protection during natural LASV infection}


In LF patients, the development of LASV antibodies are not associated with viral clearance, survival, or positive prognosis. High viremia, and high IgM and IgG titers often coexist in LF patients. At the time of hospital admission, LASV antibodies against NP or GPs were not associated with survival or positive prognosis. In contrast, patients simultaneously containing LASV antigen and specific IgM antibodies had significantly higher chances ( $>4$ times) to die in comparison with patients with IgM antibodies alone $[143,153]$. In some surviving patients, LASV IgM antibodies can persist for a prolonged time, suggesting that a broken $\operatorname{lgM} / \lg G$ switch is a part of pathogenesis $[143,154]$. In most cases of natural LASV infection, humoral responses (predominantly against LASV NP) have a short lifetime, and antibody titers decline below detectable levels within a few months $[155,156]$. In clinical trials, human convalescent plasma did not protect recipients [138]. Although LASV humoral responses do not play a role in controlling LASV during natural infection, human LASV antibody generated from B cells of LASVinfected individuals can be useful for post-exposure treatment $[157,158]$. These neutralizing antibodies locked LASV glycoprotein complex in a pre-fusion conformation and prevented the rearrangements required for interaction with LAMP1 binding and membrane fusion.

There is a widely accepted perception in the field that protective immunity during natural LASV infection in healthy individuals is predominantly associated with T cell responses [59, 159-161]. In long-term LF survivors, and in experimentally infected NHPs, early and robust virus-specific T cell responses correlated with recovery and protection [15, 141, 162-164]. Consistent with these findings, depletion of CD8+ T cells completely abolished the protection of vaccinated animals, whereas CD4+ T cell depletion only resulted in partial protection $[165,166]$. LASV specific protective CD8+ and CD4+ T-cell subsets were poly-functional, cross-reactive and cross-protective [17, 165-168]. Likewise, in a nurse who acquired LF in Togo, and was treated at 
Emory University Hospital, recovery was consistent with the development of long-term polyfunctional LASV-specific T cell responses [169].

LASV survivors had strong CD4+ T cell proliferative responses against LASV NP and GP2 epitopes $[163,164]$. Importantly, these responses were not only LASV strain-specific but were also broadly cross-reactive, potentially contributing to protection against heterologous LASV strains. These observations are in line with previous results, which documented the role of LASV NP and GP2 epitopes in induction of CD4+ T cell-based cross-reactivity and cross-protection in NHP and rodent models $[98,170]$. NP is the major structural factor of the arenavirus transcription/replication machinery complex, and is synthesized during early stages of the infection, prior to GPC. Together, these studies indicate that early, strong anti-NP immunity is crucial for effective viral control and vector immunogenicity [171, 172].

\section{Rationally designed LASV vaccine candidates}

Epidemiological evidence indicates that LF survivors acquire long-term protection against manifested LF disease, indicating that a live attenuated virus (LAV) platform represents the most effective approach to control LASV. Methods used in the past to create very effective LAV vaccines against yellow fever, polio, measles, mumps and other infections, are generally not preferred under current regulatory environment and guidelines, due to the intractability of generating attenuated strains by serial passaging. However, remarkable advances in biotechnology and molecular virology have brought renewed interest to the new generation of LAV vaccines. These vaccines are safe, efficacious and cost-effective (e.g., the human-rhesus reassortant vaccine against rotavirus infection, RotaShield, licensed for use in the US in children at 2-6 months of age) [173]. 
The safety and efficacy of several LASV vaccine candidates based on vaccinia virus [15, 127, 174], MOPV [16-18], alphavirus replicons [175, 176], VSV $[167,177]$, and yellow fever 17D $[178,179]$ vectors were tested in NHPs (Table 1$)$. The major features of these vaccine candidates and new approaches based on LASV reverse genetics were discussed recently in several reviews $[59,161,180-182]$. This subsection will focus on the most challenging areas of LASV vaccine research and development: addressing LASV heterogeneity, correlates of protection, safety in immunocompromised individuals, and regulatory pathways.

\section{LASV antigens required for induction of broad cross-reactive and long-lasting protective} immunity

The first experimental vector-based LASV vaccine expressed NP antigen of LASV/GA391/NIG/96/H (clade III) in vaccinia virus (Vv, Lister strain) [127]. This vaccine did not induce detectable LASV antibodies before challenge, but completely protected Hartley guinea pigs against fatal LF disease [127]. All other experimental LASV vaccines were designed based on LASV/Josiah (clade IV). In strain 13 guinea pigs, 94\% and 74\% of animals vaccinated with recombinant Vv (strain NYBH) expressing LASV/JOS NP or GPC were protected against homologous challenge, respectively [183]. Similarly, LASV/JOS NP expressed via alphavirus RNA replicons fully protected strain 13 guinea pigs against fatal LF disease [175]. In contrast, in the same challenge model, LASV NP expressed in an rVSV vector provided only partial protection against LASV/JOS [128]. 
Table 1. Contributions of anti-NP and-GPC adaptive immune responses in the protection of guinea pig and NHP challenge models of Lassa fever

\begin{tabular}{|c|c|c|c|c|c|c|}
\hline $\begin{array}{l}\text { Vaccine } \\
\text { Vector, } \\
\text { Dose, Route }\end{array}$ & Antigen/Challenge & $\begin{array}{l}\text { Animal } \\
\text { Model }\end{array}$ & $\begin{array}{l}\text { Protection/ } \\
\text { Viremia }\end{array}$ & CMI & $\begin{array}{l}\text { Humoral } \\
\text { immunity }\end{array}$ & Ref \\
\hline $\begin{array}{l}\text { Vv, Lister, } \\
1 \times 10^{7.5} \mathrm{PFU}, \\
\text { single dose, }\end{array}$ & $\begin{array}{l}\text { NP, GA391 } \\
\text { (III)/GA391 }\end{array}$ & Hartley GPs & $100 \% /-$ & CMI? & No nAbs & [127] \\
\hline $\begin{array}{l}\text { Vv, NYBN, } \\
\text { single dose }\end{array}$ & NP, Josiah/JOS(IV) & STR13GPS & $94 \% /+$ & CMI? & No nAbs & [183] \\
\hline $\begin{array}{l}\text { Vv, NYBN, } \\
\text { single dose }\end{array}$ & GPC, JOS/JOS & STR13GPS & $74-100 /+$ & CMI? & No nAbs & $\begin{array}{l}{[174,} \\
183]\end{array}$ \\
\hline $\begin{array}{l}\text { LASV Z-158 } \\
1 \times 10^{3.7} \text { PFU, } \\
\text { single dose }\end{array}$ & $\begin{array}{l}\text { All Z158 } \\
\text { antigens/JOS }\end{array}$ & STR13GPs & $100 \% /-$ & $\begin{array}{l}\text { CMI, } \\
\text { spleen } \\
\text { transfer } \\
\text { exp. }\end{array}$ & $\begin{array}{l}\text { Immune } \\
\text { plasma } \\
\text { did not } \\
\text { protect }\end{array}$ & [122] \\
\hline $\begin{array}{l}\text { VEEV-RNA } \\
\text { replicon, } \\
1 \times 10^{7} \mathrm{IU}, 3 \\
\text { injections }\end{array}$ & $\begin{array}{l}\mathrm{NP} \\
\mathrm{GPC} \\
\mathrm{NP}+\mathrm{GPC}, \mathrm{JOS} / \mathrm{JOS}\end{array}$ & STR13GPS & $\begin{array}{l}100 \% /- \\
100 \% /- \\
100 \%-\end{array}$ & $\mathrm{CMI}$ & $\begin{array}{l}\text { Immune } \\
\text { plasma } \\
\text { did not } \\
\text { protect }\end{array}$ & [175] \\
\hline $\begin{array}{l}\text { MOPV/LASV } \\
\text { (ML29), } \\
1 \times 10^{3}, \text { single } \\
\text { dose }\end{array}$ & $\begin{array}{l}\text { NP\&GPC, JOS/JOS, } \\
803213(\text { II) }\end{array}$ & STR13GPS & $\begin{array}{l}100 \% /- \text { (for } \\
\text { JOS } \\
\text { challenge) } \\
100 \% /+ \\
\text { (for } 803213 \\
\text { challenge }\end{array}$ & $\mathrm{CMI}$ & $\begin{array}{l}\text { No nAbs, } \\
\text { IgG Abs }\end{array}$ & $\begin{array}{l}17, \\
125]\end{array}$ \\
\hline $\begin{array}{l}\text { YF17D/LASV, } \\
1 \times 10^{5} \mathrm{PFU}, \\
\text { single dose }\end{array}$ & GPC, JOS/JOS & STR13GPS & $80 \% /+/-$ & CMI? & $\begin{array}{l}\text { No nAbs, } \\
\text { IgG Abs }\end{array}$ & [178] \\
\hline $\begin{array}{l}\text { rVSV/LASV, } \\
1 \times 10^{6} \mathrm{PFU}, \\
\text { single dose }\end{array}$ & $\begin{array}{l}\text { GPC, JOS/JOS, Z- } \\
132 \text {, Soromba-R }\end{array}$ & STR13GPs & $\begin{array}{l}100 \% \\
\text { (against } \\
\text { JOS and Z- } \\
132 \\
\text { challenge) }\end{array}$ & ND & $\begin{array}{l}\text { No nAbs, } \\
\text { IgG Abs }\end{array}$ & $\begin{array}{l}128, \\
184]\end{array}$ \\
\hline $\begin{array}{l}\text { rVSV/LASV, } \\
1 \times 10^{5} \mathrm{PFU}, \\
\text { single dose }\end{array}$ & NP, JOS/JOS & STR13GPS & $66.6 \%$ & ND & ND & [128] \\
\hline $\begin{array}{l}\text { DNA, } 300 \mu \mathrm{g}, \\
3 \text { injections, } \\
\text { IMEP or IDEP }\end{array}$ & GPC, JOS/JOS & STR13GPS & $\begin{array}{l}80- \\
100 \% /+/-\end{array}$ & CMI? & $\begin{array}{l}\text { nAbs, low } \\
\text { titers }\end{array}$ & [185] \\
\hline $\begin{array}{l}\text { Rc- } \\
\text { LASSARABV, } \\
1 \times 10^{6} \mathrm{FFU}, \\
\text { single dose }\end{array}$ & GPC, JOS/JOS-GP & Hartley GPs & $40 \%$ & ND & $\begin{array}{l}\text { No nAbs, } \\
\text { IgG Abs }\end{array}$ & [184] \\
\hline $\begin{array}{l}\text { LASSARAB, } \\
\text { killed, with }\end{array}$ & GPC, JOS/JOS-GP & Hartley GPs & $80 \% /+++$ & & No nAbs, & [184] \\
\hline
\end{tabular}




\begin{tabular}{|c|c|c|c|c|c|c|}
\hline $\begin{array}{l}\text { TLR4 agonist, } \\
3 \text { injections }\end{array}$ & & & & & $\begin{array}{l}\text { non-nAbs } \\
\text { involved } \\
\text { in ADCC }\end{array}$ & \\
\hline $\begin{array}{l}\text { LASV, VRP, } \\
1 \times 10^{7}, \mathrm{SC}\end{array}$ & $\begin{array}{l}\text { All LASV/JOS } \\
\text { proteoms } / / J O S\end{array}$ & STR13GPS & $100 \% /-$ & CMI & $\begin{array}{l}\text { No IgM } \\
\text { or IgG } \\
\text { before } \\
\text { challenge }\end{array}$ & $\begin{array}{l}186, \\
187]\end{array}$ \\
\hline $\begin{array}{l}\text { LASV/JOS, } \\
\text { killed, } \\
\text { multiple } \\
\text { injections } \\
\end{array}$ & $\begin{array}{l}\text { All LASV } \\
\text { proteoms/JOS }\end{array}$ & $\begin{array}{l}\text { NHPs } \\
\text { (cynos) }\end{array}$ & $0 \%$ & & $\begin{array}{l}\text { No nAbs, } \\
\text { IgG Abs } \\
\text { (anti-NP, } \\
\text {-GPC) }\end{array}$ & [188] \\
\hline $\begin{array}{l}\text { MOPV, } \\
\text { single dose, }\end{array}$ & $\begin{array}{l}\text { All MOPV } \\
\text { proteoms/JOS }\end{array}$ & $\begin{array}{l}\text { NHPs } \\
\text { (rhesus) }\end{array}$ & $100 \% /-$ & ND & ND & $\begin{array}{l}15, \\
73, \\
74]\end{array}$ \\
\hline $\begin{array}{l}\text { Vv, NYBN, } \\
\text { single dose }\end{array}$ & $\begin{array}{l}\text { NP } \\
\text { GPC } \\
\text { NP\&GPC,JOS/JOS }\end{array}$ & $\begin{array}{l}\text { NHPs } \\
\text { (rhesus, } \\
\text { cynos) }\end{array}$ & $\begin{array}{l}27 \% \\
85 \% /++ \\
100 \% /+\end{array}$ & CMI & & {$[15]$} \\
\hline $\begin{array}{l}\text { rVSV, } 1 \times 10^{6} \\
\text { PFU, single } \\
\text { dose }\end{array}$ & GPC, JOS/JOS & $\begin{array}{l}\text { NHPs } \\
\text { (cynos) }\end{array}$ & $100 \% /++$ & & nAbs & [167] \\
\hline $\begin{array}{l}\text { ML29, } 1 \times 10^{3} \\
\text { PFU, single } \\
\text { dose }\end{array}$ & $\begin{array}{l}\text { GPC\&NP, JOS/JOS, } \\
\text { LCMV-WE }\end{array}$ & $\begin{array}{l}\text { NHPs } \\
\text { (rhesus, } \\
\text { marmosets) }\end{array}$ & $\begin{array}{l}100 \% /- \\
\text { (against } \\
\text { LASV/JOS } \\
\text { and LCMV- } \\
\text { WE }\end{array}$ & CMI & No nAbs & $\begin{array}{l}{[16,} \\
17, \\
161, \\
168]\end{array}$ \\
\hline $\begin{array}{l}\text { MOPEVAC }{ }_{\text {LAS, }} \\
\text { single dose, } \\
6 \times 10^{6} \mathrm{FFU}, \\
\text { SC }\end{array}$ & GPC, JOS/JOS & $\begin{array}{l}\text { NHPs } \\
\text { (cynos) }\end{array}$ & $100 \%$ & CMI & nAbs & [75] \\
\hline $\begin{array}{l}\text { DNA, } 10 \mathrm{mg} \text {, } \\
4 \text { sites, } 2-3 \\
\text { injections, } \\
\text { IDEP }\end{array}$ & $\begin{array}{l}\text { GPC, codon } \\
\text { optimized, JOS/JOS }\end{array}$ & $\begin{array}{l}\text { NHPs } \\
\text { (cynos) }\end{array}$ & $100 \% /-$ & CMI & nAbs, IgG & $\begin{array}{l}{[189,} \\
190]\end{array}$ \\
\hline
\end{tabular}

Efficacy trials in NHPs (Macaca mulatta and M. fascicularis) with rVv expressing different LASV genes documented that both GPC subunits, GP1 and GP2, are required for protection $[15,191]$. In contrast to results in guinea pigs, vaccination with rVv-NP protected only 3 of 15 NHPs (22\%). Nevertheless, the highest level of the protection was observed when NHPs were vaccinated with rVv expressing both genes, NP\&GPC, simultaneously [15]. In addition, while $V v$ vectored vaccine formulations failed to induced sterilizing immunity, the lowest level of 
post-challenge viremia was observed in NHPs immunized with VV-NP\&GPC. Similar to guinea pigs, protection in NHPs was not associated with humoral immune responses [15, 156].

These results indicate that expression vectors and animal challenge models have an effect on the results of vaccine efficacy trials. In terms of antigen formulation, there is a consensus that expression of LASV GPC is required and sufficient for solid protection. While antiNP immunity in rodent models is highly protective, it is still not enough to protect NHPs, suggesting different contributions of anti-NP immunity in viral control between rodents and NHPs. The positive impact of anti-NP immunity on viral control at an early stage of the infection is well anticipated because NP plays the central role in transcription/replication [192]. NP together with L protein are the two minimal trans-acting factors required to rescue LCMV and LASV from recombinant cDNAs $[193,194]$. Detection of broad cross-reactive anti-NP CD4+ T cells in LF survivors suggests contribution of these cells in long-term protection against LF caused by LASV strains from different clades. Among LASV advanced vaccine candidates (tested in NHPs), only ML29 has the ability to induce LASV-specific anti-NP and anti-GPC immune responses (Table 1) [17].

As expected, LASV/JOS GPC-based experimental vaccines were protective against challenge with the homologous JOS strain or strains from the same clade but from different geographical areas [128]. While LASV/JOS represents the largest collection of LASV strains in West Africa, the most diverse LASV strains from clade I-III circulate in Nigeria $[53,56]$. The ML29 vaccine expressing both JOS-derived genes, NP and GPC, fully protected strain 13 guinea pigs and NHPs against LASV/JOS(IV) and LASV/803213(II) [125]. Taking into consideration the close phylogenetic relationship between clade IV and III, the ML29-induced protection against LASV strains from clade III is very likely. 
LASV/JOS causes fatal disease mimicking human LF in strain 13 guinea pigs and NHP species, rhesus, cynomolgous, and African green monkeys, and in marmosets [122, 123, 126, 195]. Infection of capuchin and squirrel monkeys resulted in mild disease. Strain 13 guinea pigs and cynomolgous monkeys are the most widely used "FDA-validated" LASV/JOS challenge models. Animal studies of Nigerian LASV strains are limited. The LASV/GA391 from clade III is lethal for Hartley guinea pigs [127]. The Nigerian prototypic strain 803213 from clade II causes LF-like fatal disease in strain 13 guinea pigs and marmosets. However, the prototypic strain LP/NIG69/H (clade I), which occupies the bottom of LASV phylogenetic tree, did not induce fatal disease in strain 13 guinea pigs, complicating the evaluation of efficacy of experimental LASV vaccines [128]. There is no information on the sensitivity of NHPs to LASV/LP infection.

Taking into consideration the distant genetic relationship between clades I and IV, there is a concern that LASV/JOS-based vaccines will not provide full protection against LASV/LP challenge. In this case, LASV/LP antigens (at least GPC) need to be included in the vaccine formulation. Both advanced vaccine candidates, rVSVDG/LASV-GPC and ML29, have powerful reverse genetics tools capable of adding the expression of GPC from LASV/LP. Successful expression of two arenaviral glycoproteins including LASV GPC from clade I and IV was also reported in an alphavirus RNA replicon system $[161,175,196]$. The development and standardization of reliable challenge models for Nigerian strains of LASV is urgently needed to secure clinical development of successful LASV vaccine in West Africa.

\section{Vector Platform-based LASV vaccine candidates}

In contrast to EBOV and other emerging pathogens, which cause unpredictable outbreaks with limited numbers of human cases, LF is a common disease in West Africa. In emergency and non-emergency situations, regulatory agencies (the US Food and Drug 
Administration, FDA; the European Medicines Agency, EMA) will require LASV vaccine efficacy trials in humans. Both agencies can act as Reference National Regulatory Authorities (NRAs) for the purposes of WHO pre-qualification, and/or assist other NRAs in their evaluation of the safety and effectiveness of the vaccine. Further, both NRAs will issue a Certificate of Pharmaceutical Product (CPP) upon which some countries will then use as the basis for their review of a vaccine candidate. In the US, the FDA encourages the path to Biological License Application (BLA) and licensure even if disease is not endemic in the US. Vaccine development in the US follows the same general path as other drugs intended for human use, and is evaluated during clinical development under an Investigational New Drug (IND). Sponsors of vaccines for diseases endemic in geographies outside the US have access to a variety of regulatory mechanisms of acceleration, such as "Fast Track" designation, and others that address unmet medical needs.

In the EU, the EMA is responsible for the scientific evaluation of marketing applications, with the Committee for Medicinal Products for Human Use (CHMP) holding responsibility for human medicines, including vaccines. The EMA has a procedure (Article 58) that allows the agency to render an opinion in cooperation with the WHO for the evaluation of drugs intended for use outside the European Community.

The history of the development and regulatory pathway of Candid 1, the first arenaviral vaccine (manufactured and licensed in Argentina) [197], can be a valuable guideline for LASV vaccine clinical development. Candid 1 significantly reduced numbers of arenaviral HF cases from 3,500 to $30-50$ per year. In the US, Candid 1 is still under IND status and it is not "approvable" due to missing records of passages at the early stages of attenuation. The redesigned, "rescue" of recombinant Candid 1 from cDNA clones provides hope that a version of Candid 1 will get FDA approval in the future [198]. 
Currently, two vaccine candidates, ML29 and rVSVDG/LASV-GPC, have been considered for "accelerated" development [199], and the rVSV-based LASV vaccine is scheduled to be tested in a Phase I trial in 2017-2018 [166]. There is a solid rationale to assess safety and dosedependent immune responses (phase II) in volunteers in endemic and non-endemic areas before getting the national authorization for efficacy trials. Nigeria has the most developed medical infrastructure in Sub-Sahara Africa, and several clinical research centers are already involved in LASV international research programs $[53,157,200]$. Together with Guinea and other African countries, Nigeria is a member of African Vaccine Manufacturing Initiative, with a mission to promote the establishment of sustainable human vaccine manufacturing capacity in Africa.

While the clinical development of LASV vaccines in Africa is feasible, several risk factors have to be taken into consideration and addressed. First, contract research organizations (CROs) managing all aspects of clinical development under "good clinical practices" are not well represented in West Africa. Family Health International (FHI360, www.fhi360.org) with regional medical research experience, including development of EBOV vaccines, can be considered as a potential partner for clinical trial-related operations. Second, to secure proper enrollment, a clear description of benefits and potential risks for healthy subjects needs to be provided. Third, the regulatory environment and the broad range of costs per patient (from $\$ 2,500-5,000$ in Nigeria to $\$ 8,500$ in Sierra Lone) is additional factor of uncertainty.

In "first-in-human" safety trials, 18-55 years old subjects with a healthy immune system will be enrolled, with well-defined inclusion and exclusion criteria. There is a reasonable expectation that dose-dependent immunogenicity trials would be conducted in endemic areas, with the possible enrollment of healthy subjects previously exposed to LASV infection. Prior immunity will potentially affect vaccine-induced immunity, and will require sub-group analysis 
to assess immunogenicity in naïve recipients receiving low and high doses of the vaccine. Safety and immunogenicity of LASV in HIV-1 positive subjects is a solid rationale for separate trials after the basic safety and immunogenicity profile of the vaccine in healthy individuals is defined.

The "ideal" LASV vaccine has to be developed for the general population of West Africa including children from different age groups. Pediatric studies in the US and the EU are subject to special provisions of the clinical trial regulation, and a Pediatric Investigational Plan (PIP) would normally need to be developed and authorized. Currently, there is no clear regulatory pathway describing how to incorporate a PIP in LASV vaccine development. As such, a PIP should be prepared and discussed with the FDA and the EMA during the pre-IND and Scientific Advice meetings.

One of the goals of the recently launched Coalition for Epidemic Preparedness Innovations (CEPI) is to optimize the approval process for vaccines against emerging infectious diseases, and make this process significantly shorter, transparent, and more effective [201-203]. There is a hope that the CEPI initiative will be a game changer, and will provide innovative tools to proactively develop and manufacture vaccines against epidemic infectious diseases. Accelerated clinical development of LASV vaccine is one of the CEPI priorities for the next five years.

\section{Conclusion}

LASV infection is a growing threat for the populations of West African countries and non-endemic areas. Recent evidence of LASV expansion suggests that all countries in the tropical wooded savanna areas between Nigeria and Senegal can be considered a LASV endemic zone. Improvement in molecular-based detection of LASV and species-identification of small mammals discovered new LASV-related species and new LASV natural hosts. Taking into 
consideration the high genetic and biological diversity, the taxonomic status of LASV as a single species may be reevaluated in coming years.

Our knowledge of how LASV interacts with susceptible cells has been significantly improved. Although sophisticatedly-glycosylated $\alpha$-DG is the major LASV cellular receptor, some C-type lectins and TAM family proteins can also be involved in LASV entry in cells lacking functional $\alpha$-DG (e.g., mature hepatocytes). The requirement of the intracellular receptor LAMP1 for LASV entry provided evidence of similarity with EBOV entry. Despite differences in morphology and vesicular trafficking, both viruses use glycoprotein GP1 sub-unit to initiate interactions with host receptors and the required intracellular receptor switch, to NPC1 (for EBOV) and to LAMP1 (for LASV) for cell entry. The surface of both viruses are heavily glycosylated to prevent interaction with specific antibodies, a common mechanism of immune control evasion. Intriguingly, the GP2 sub-unit of newly discovered reptilian arenaviruses are highly similar to those of EBOV suggesting a possible ancestor interaction between arena- and filoviruses.

A NHP model continues to be the major source of our knowledge on LF pathogenesis. In this model, LASV strains from different endemic areas demonstrated a range of clinical manifestations from mild infections with low lethality to severe hemorrhagic fevers with fatal outcome, potentially mimicking differences in clinical forms of human LF. With obvious limitations, murine models can still be helpful to study certain steps of the acute disease (e.g., liver involvement), LF sequelae (e.g., sensorineural hearing deficiency), and immunogenicity of experimental LASV vaccines (vaccine potency). Some evidence of LASV-induced immunopathology observed in immunocompromised and chimeric mice seems to be associated with the inherent phenotype of LASV (and LCMV) in mice/rodents. 
With improvements in virus and antibody detection, the negligible role of humoral responses in recovery and protection during the natural course of LASV infection was additionally documented. Nevertheless, monoclonal antibodies specific for LASV GPC were successfully cloned from memory B cells of the survivors, and demonstrated therapeutic activities in a NHPs lethal challenge model. These antibodies can potentially be used alone or in combination with ribavirin for the treatment of LF patients at the time of hospital admission.

A new generation of replication competent vaccines represents the most feasible approach to control LASV. At least two vaccine candidates, rVSVAG/LASV-GPC and reassortant ML29, have demonstrated safety and efficacy in NHPs, and are well positioned for rapid clinical development. The new initiative, Coalition for Epidemic Preparedness Innovation (CEPI), has been recently established for proactive development of effective vaccine against epidemic infections. The CEPI included development of LASV vaccines in its 2017-2021 business plan, with the goal to develop vaccines through phase II for further stockpiling for emergency use and/or phase III efficacy trials. In case of LASV, "emergency use" is permanent since this infection is endemic for West Africa. This suggests that once regulatory issues for CEPI vaccines are settled, the lead LASV vaccines will be tested in multicenter efficacy trials in major endemic areas of Nigeria and West African countries. 


\section{CHAPTER III}

\section{ATTENUATED REPLICATION OF LASSA VIRUS VACCINE CANDIDATE ML29 IN STAT-1-/- MICE ${ }^{1}$}

\section{Introduction}

Lassa virus (LASV) is a highly prevalent arenavirus in West Africa, where it infects several hundred thousand individuals annually. This results in a large number of Lassa fever (LF) cases that are associated with high morbidity and mortality rates $[80,133]$. The natural LASV reservoir is the rodent Mastomys natalensis, which is widely distributed throughout sub-Saharan Africa. The area where LASV is endemic covers large regions of West Africa [61] putting a population of up to 200 million people at risk for infection [60]. Furthermore, there is evidence that LASV endemic regions are expanding [62]. The high degree of LASV genetic diversity $[53,56]$ likely contributes to underestimates of its prevalence [65]. With the exception of Dengue fever, LF has the greatest estimated global burden among all viral hemorrhagic fevers (HFs) [59]. LF outbreaks are associated with morality rates as high as 60\%, as documented during the $2015-2016$ outbreak in Nigeria [147]. The currently ongoing 2018 outbreak is the worst on record [205]. There is no FDA-approved LASV vaccine or therapeutic. Treatment options are limited to offlabel ribavirin with varying degrees of success. In 2017-2018, the WHO included LASV on the top priority pathogens list and issued a Target Product Profile (TPP) for LASV vaccine development $[64,206,207]$.

\footnotetext{
${ }^{1}$ This chapter is adapted from work published in Pathogens, 2019, Volume 8, Issue 1 with the same title under a Creative Commons Attribution (CC BY) license [204].
} 
LASV belongs to the Old World (OW) group (former lymphocytic choriomeningitis virus, LCMV-LASV sero-complex) of the genus Mammarenavirus of the Arenaviridae family [45]. The LASV genome has two RNA segments that each encode two open reading frames in opposite polarities, separated by a structured intergenic region. The L RNA encodes for a large $L$ protein, which functions as a RNA-dependent RNA polymerase (RdRp), and a RING finger protein Z, which comprises the matrix of virions. The S RNA encodes for a nucleoprotein (NP) which tightly associates with viral RNA and a glycoprotein precursor (GPC) that is processed into three subunits: a stable signal peptide, SSP; a receptor-binding GP1; and a transmembrane GP2 which mediates fusion with cell membranes. All three glycoprotein subunits associate to form clubshaped features on the surface of pleomorphic viral particles, which vary in the diameter from 50 to $300 \mathrm{~nm}$. In addition to LASV, the OW group includes genetically related non-pathogenic viruses: Mopeia (MOPV), Morogoro (MORV), Gairo (GAIV) and Luna (LUNV) hosted by the same rodents [69-72]. The New World (NW) mammalian arenaviruses (former Tacaribe virus, TCRV, sero-complex) comprise viruses which circulate in Americas, and include the causative agents of South American HFs [208].

Reassortant studies using genetically-related mammalian arenaviruses with different pathogenic potential demonstrated that the $L$ gene is responsible for high levels of virus replication in vivo, and is associated with acute disease in experimental animals $[209,210]$. Coinfection of cells with LASV and MOPV resulted in generation of MOPV/LASV reassortants [18, 211]. One of the rationally selected clones, ML29, carrying the L RNA from MOPV and the S RNA from the Josiah strain of LASV (LASV/JOS), is a promising LASV vaccine candidate $[17,18,199]$.

While MOPV L RNA is the major driving force of ML29 attenuation, eighteen mutations incorporated in the ML29 genome during in vitro selection additionally seem to contribute to the attenuation of ML29 $[17,212]$. Indeed, transcriptome profiling of human peripheral blood 
mononuclear cells (hPBMC) exposed to LASV or ML29 exhibited distinct molecular signatures that can be useful biomarkers of pathogenicity and protection [16, 151]. Similar studies comparing MOPV and ML29 infected hPBMC revealed that gene expression patterns in mock and ML29 infected hPBMC clustered together and differed from the expression pattern observed in MOPV infected hPBMC [16].

Recently, recombinant ML29 (rML29) was rescued from cDNA clones and demonstrated the same features as a biological ML29 (bML29) [213]. Reverse genetics provides a powerful tool for elucidation of the individual contributions of ML29-specific mutations to attenuation. However, the absence of a reliable small animal model for LASV pathogenicity is a major obstacle for the field. LASV, as well as other mammalian arenaviruses, stimulate antiviral immunity differently in natural rodent hosts and in humans and non-human primates, NHPs [15, $123,159]$. While some pathogenic features of human LF can be observed in strain 13 guinea pigs $[122,124,214]$, there is a poor correlation between clinical outcome of LF in humans and virulence of LASV in guinea pigs [121]. Results of LASV vaccination/challenge studies in strain 13 guinea pigs are not reproducible in NHPs $[15,175,183]$. In addition, strain 13 guinea pigs are no longer commercially available and difficult to breed.

Rodent models remain useful to study some mechanisms of LF pathogenesis and protective immune responses, and to provide valuable information during pre-clinical development of vaccine candidates $[123,215]$. Previous research has documented that mice lacking a functional STAT1 pathway are highly susceptible to LASV infection, and develop fatal disease with some pathological features that mimic human LF [198]. Notably, human LASV isolates from fatal LF cases in Sierra Leone induced clinically similar fatal disease including high viral load in blood and visceral organs of STAT-1 ${ }^{--}$mice after intraperitoneal inoculation (i.p.). In contrast, LASV isolated from a non-lethal human case induced disease with moderate mortality. 
Surviving mice developed hearing loss that mimicked the sensorineural hearing loss (SNHL) commonly observed in LF patients during convalescence [216].

In this study, we provided additional evidence of deep ML29 attenuation by modeling the LASV infection protocol for STAT ${ }^{-/}$mice as a means to assess the safety profile of ML29 (bML29 or rML29) in comparison with MOPV. We also generated ML29 stocks enriched with interfering particles (IPs) produced by ML29-persisitently infected cells. The growing body of evidence indicates that, during natural infection, IPs are critically involved in the modulation of viral load, innate immune responses, disease outcome, and contribute to the evolutionary persistence of viruses [217-219]. An accumulation of IPs with genome deletions have been documented during the production of live-attenuated polio [220], influenza [221, 222] and measles [223-225] vaccines, and was associated with modulating vaccine potency and immunostimulatory properties. Here, we demonstrated that STAT ${ }^{-1-}$ mice are a susceptible model capable of discriminating between viruses with different levels of attenuation, MOPV and ML29. ML29 IPs were completely attenuated in these mice, enhancing immunogenic features of ML29. The broad cross-protection activity of ML29 IPs and their intrinsic adjuvant features can be potentially used for rational formulation of a pan-LASV ML29-based vaccine by developing vaccine manufacturing protocols to optimize the ratio between infectious ML29 and IPs.

\section{Results}

\subsection{Generation of interfering particles (IPs) by ML29-persisitently infected cells}

Arenavirus IPs can be generated at high multiplicity of infection (MOI) by serial infection of fresh tissue cultures with undiluted culture medium harvested from previous passages. Alternatively, initially infected cells can be subjected to serial passages. Both methods resulted in the accumulation of IPs. Earlier studies suggested a contribution of IPs in the establishment 
and/or maintenance of LCMV persistent infection in vitro and in vivo, and in the inhibition of virus-induced immunopathology in mice [226-232]. The protocol previously applied for the generation of LASV IPs in cell cultures was used to establish ML29 persistent infection of Vero cells. In line with previous observations [233-235], passages of ML29-infected cells, either infected with bML29 or with rML29, resulted in the gradual decline of infectious particles released in culture medium. As seen in Figure 1, the infectious titers of ML29 released into the medium of persistently infected Vero cells (Vero/ML29) was reduced almost 100-fold after 10 passages, and was under detectable levels after 15 passages. Vero/ML29 cells strongly interfered with replication of homologous (ML29) or genetically related LASV/JOS, LCMV, and MOPV and did not generate infectious plaques after super infection of Vero/ML29 with these viruses. Meanwhile, neither the replication of TCRV, a NW arenavirus, nor the non-related Ebola virus was affected in these cells. ML29 IPs released from persistently infected Vero/ML29 cells were able to suppress replication of homologous virus in a dose-dependent manner (Figure 1f).

While the replication of ML29 or LASV was not detectable in ML29/Vero cells after passage 10, viral proteins were detected in these cells by immunofocus (IF) assay (Figure 1c). In contrast to infectious "plaques", this assay detects cells expressing virus antigens stained by specific antibodies. With an increasing number of passages, ML29-infected cells lost their ability to produce plaques after homologous superinfection, but were strongly positive for ML29specific antigens as demonstrated by quantitated analyses (Figure 1c; Figure 6). Differences in the morphology of IF foci between naïve and Vero/ML29 cells after infection and superinfection with ML29, respectively, seems to be related to differences in the expression of NP and GP proteins in different passages of Vero/ML29.

Viral RNA load in Vero/ML29 cells, as assessed by quantitative qRT-PCR targeting the NP gene, declined about 10 fold during the first five passages and persisted approximately at the 
same levels during subsequent passages of Vero/ML29 (Figure 1b). Viral RNA load remained at a similar level through 50 passages (data not shown.) Our attempts to detect genomic RNA deletions, either in infected cells or in virions released from infected cells, using RT-PCR failed (not shown, see Discussion).

\subsection{MOPV and attenuated reassortant ML29 induce experimental disease with different clinical} manifestations and outcome in STAT-1\% mice

In STAT1 $\%$ mice, LASV infection resulted in experimental disease with the outcome depending on the pathogenic potential of the LASV isolate [216]. Based on these observations, we tested the attenuation of MOPV and ML29 in these mice. Using the LASV infection protocol, three groups of mice $(n=9)$ were IP inoculated with MOPV, ML29 and ML29P50 (IPs produced by Vero/ML29, passage 50). MOPV infection resulted in poor grooming, lethargy and signs of dehydration starting around day 8-post infection. These symptoms aggravated, and the mice gradually lost weight, and experienced a drop in body temperature. All animals in this group met euthanasia criteria within 11-17 days following infection (Figure 2). In ML29 infected STAT1 ${ }^{-/}$ mice, the symptoms started to improve at the late stage of the infection. Body temperature quickly recovered after day 10 and $33 \%$ of mice survived at day 21 . No clinical signs were observed in the ML29P50 group. These mice gained weight at the end of the observation period and their temperature fluctuated within a normal range.

As a control, wild type mice with an identical genetic background were i.p. inoculated with MOPV, ML29 and ML29P50. The mice were successfully infected with these viruses as was documented by detection of viral RNA in tested tissues (Figure 7). However, in the wild type mice, the replication of the viruses was well controlled and viral RNA was barely detectable at 
day 21. As expected, infection did not induce clinical manifestations in any of the wild type study groups, and these mice gained weight at the end of the 21 days observation period.

\subsection{Replication of MOPV and ML29 in tissues of STAT- $\%$ mice: no viremia and infectious virus in} tissues of ML29P50 infected mice

High viremia, infectious titers, and viral RNA loads were associated with replication of MOPV in tissues of STAT-1 ${ }^{--}$mice as assessed by infectious plaque assay and qRT-PCR (Figure 2). At the peak of clinical manifestation, day 7-10, viremia and viral load in the liver reached more than $1 \times 10^{7} \mathrm{PFU} / \mathrm{ml}$ or PFU/g, respectively. RNAscope in situ hybridization confirmed extensive liver infection with strong signals associated with hepatocytes and endothelial cells (Figure 3, arrowed). In contrast, replication of ML29 was more attenuated in STAT-1 ${ }^{\%}$ mice. Viremia and viral load in the liver was 3-logs lower in these mice compared with MOPV infection, and only a few weakly-positive hepatocytes were seen in ML29-infected liver samples. In brain tissue, replication of infectious ML29 was transiently detected at early and late stages of infection. Replication of infectious ML29P50 in STAT-1 ${ }^{-}$mice was below detectable levels in blood, liver, and brain. However, replication of ML29P50 was detected in tissues by viral RNA-based assays, qRT-PCR, and RNAscope in situ hybridization. As seen in Figure $2 \mathrm{~g}-\mathrm{i}$, at early stage of the infection, relative levels of ML29P50 viral RNA in tissues were lower than MOPV and ML29 viral RNAs. ML29P50 was barely detectable in the tissues at late stage of the infection. This indicates that ML29P50 was well controlled, an observation that is in line with the absence of clinical manifestations.

\subsection{Host responses in mice infected with MOPV and ML29}

Cell-mediated immune responses in infected mice were assessed by ELISPOT using stimulation of spleen cells isolated on day 3,7 , and 21 after infection with peptide cocktails 
derived from LASV/JOS GPC and MOPV GPC as previously described [165, 236]. At day 3, virusspecific T cells secreting IFN- $\gamma$, IL-2, or both were barely detectable in all groups of mice (not shown). By day 7 , in STAT- $1^{-/}$mice, comparable levels of IFN- $\gamma$-secreting cells ( $~ 0.4 \%$ of total spleen cells) were observed in spleens from ML29 and ML29P50 infected mice (Figure 4a). In wild type mice, ML29 and ML29P50 infection induced slightly lower T-cell responses. Notably, in wild type and STAT-1 ${ }^{--}$mice infected either with ML29 or with ML29P50, similar levels of IFN- $-\mathrm{-}$ secreting cells were induced after stimulation with GPC cocktails derived from LASV or MOPV. Infection with MOPV induced comparatively diminished T cell responses in wild type and in STAT $-1 \%$ mice, predominantly after stimulation with homologous MOPV GPC-derived peptides. After stimulation with GPC cocktails, spleen cells isolated from mice infected with ML29 and ML29P50, but not with MOPV, also secreted IL-2 cytokine, 0.1\% of total spleen cells. Cells secreting both cytokines were easily detectable at day 7 in ML29 and ML29P50 mice after stimulation with homologous antigens. In MOPV infected STAT-1\% mice, this cells population was barely detectable.

Survival of ML29P50-infected mice was clearly associated with strong cell-mediated responses. As seen in Figure 4, in spleens isolated from STAT-1\% mice on day 21 , cells secreting IFN- $\gamma$, IL2, or both were strongly upregulated after stimulation with GPC cocktails, $>0.3-0.6 \%$. In wild type mice inoculated with ML29P50, these responses had declined by this time point. Interestingly enough, in both groups of mice infected with ML29P50 humoral responses were similar (Figure 4g).

Measurement of pro-inflammatory cytokine expression in spleen of STAT-1 ${ }^{-1-}$ mice infected with MOPV revealed upregulation of IL- 6 and IL-1 $\beta$ at early stages of the infection and rapid declined at later time points while TNF- $\alpha$ was down-regulated for all viruses except the early stages of MOPV and the late stage of ML29P50 (Figure 5, Figure 8). Levels of IL-1 $\beta$ and IL-6 
mRNA in spleens of the mice infected with ML29 and ML25P50 were upregulated at day 3 and day 7 , respectively. At the later time point, the infection had minimal if any effect on TNF- $\alpha$ and IL-1 $\beta$ expression in STAT-1 ${ }^{-/}$and control mice. In MOPV-infected wild type mice, IL-6 mRNA levels had similar kinetics with those in STAT-1\%- mice, with down-regulation in the liver and spleen tissues during the infection.

\section{Discussion}

The first aim of this study was to determine if STAT-1 ${ }^{--}$mice provide a reliable small animal model to assess level of attenuation of LASV-related arenaviruses. LASV and MOPV share a common reservoir, M. natalensis, found in Western and Southeastern regions of Africa [69, 70, 237]. In Mozambique and Tanzania, areas where MOPV and MORV are prevalent, there is a marked absence of clinical cases of LF. Additionally, MOPV does not cause clinical signs in guinea pigs and NHPs [122]. These observations, combined with ability of MOPV to generate in vitro replication-competent reassortants with LASV [18], and to protect experimentally infected NHPs against fatal LF [73], suggests that MOPV is a "naturally-attenuated" genetic relative of LASV.

Studies to assess virulence of human LASV isolates in NHPs are very limited [129]. LASV isolates from LF patients with different clinical outcome showed a weak correlation between severity of human disease and virulence in guinea pigs $[121,238]$. Meanwhile, in STAT-1 ${ }^{--}$mice, outcome of the disease correlated with virulence of human LASV isolates. 4 out of 5 mice infected with a lethal isolate met euthanasia criteria between days 7 and 8 , while mice infected with nonlethal LASV isolates developed chronic disease $[198,216]$. In our experiments, MOPV infection of STAT- ${ }^{-/}$mice induced manifested disease that met euthanasia criteria 11-17 days post infection (Figure 2a). The delayed euthanasia time-point of 11-17 days versus 7-8 days, indicates some level of attenuation of MOPV infection in STAT-1 ${ }^{-/-}$mice. However, high levels of 
viremia and viral load in target tissues, comparable with those in animals infected with virulent LASV isolates [198, 216], and poor adaptive immune responses (Figure 4) resulted in aggravated disease. In contrast to MOPV, ML29 infection in STAT-1 1 mice was more attenuated, and $33 \%$ of animals survived in this group (Figure 2a). Compared to MOPV infected mice, ML29 infection notably resulted in more than a thousand-fold reduction in viremia and viral load in tissues by plaque assay (Figure $2 \mathrm{~d}-\mathrm{f}$ ). Strong T cell responses contributed to viral control in this experimental group (Figure 4). It is well documented that strong T cell-mediated immunity correlates with protection and positive clinical outcomes in experimentally infected NHPs and LF patients $[159,169,239,240]$.

The second aim of this study was to assess the contribution of IPs generated by ML29persistently infected cells to the attenuation and modulation of immune responses. Similar to many other RNA viruses, mammalian arenaviruses can generate IPs during acute infection of cells at high $\mathrm{MOI}$, or during persistent infection. The defective IPs: (i) are antigenically identical to parental viruses and contain the same structural proteins; (ii) preserve $5^{\prime}$ and $3^{\prime}$ terminal sequences of parental genome but have extensive internal deletions; (iii) can only replicate with help of standard virus; and (iv) strongly compete for replication machinery with the parental virus [178, 241-244]. Historically, arenavirus IPs were observed following in vitro infection at high $\mathrm{MOI}$, and masked the cell-killing potential of standard viruses. Earlier studies suggested the contribution of IPs in the establishment and/or maintenance of LCMV persistent infection in vitro, and in inhibition of virus-induced immunopathology in mice [226-232]. Similarly, LASV IPs generated by persistently infected cells strongly interfered with replication of standard LASV in vitro, were attenuated in $\mathrm{C} 3 \mathrm{H}$ mice, and partially protected mice against wild type LASV challenge $[233,235,245,246]$. In contrast to IPs generated from VSV, Sendai, Sindbis, and other RNA viruses, arenavirus IPs are difficult to separate from standard virions [229]. The unique 
ability of mammalian arenaviruses to incorporate host ribosomes and RNAs during the late stage of virus maturation [45] seems to be responsible for failure of density-based methods to purify arenavirus IPs. Nevertheless, LCMV defective IPs partially purified from culture medium of persistently infected cells lacked the S RNA segment [247], compared to LASV defective IPS where the L RNA segment was not detectable [234]. In line with these results, UV inactivation experiments confirmed "smaller" genomes for LCMV [228] and LASV defective IPs [234]. The LCMV L RNA segment was much less abundant and not detectable during the early stage of persistent infection. However, RNA deletions were not found among virus-specific RNA species in brain tissue of LCMV persistently infected mice. During the progression of persistence, an accumulation of terminally truncated RNA species was documented [248].

In our experiments, we were able to demonstrate the basic features of ML29 IPS generated by persistently infected cells (gradual infectivity loss during passages, antigenic identity and persistence, GP/NP ratio fluctuation, interference with homologous and closely related viruses) (Figure 1, 6). However, we failed to detect genomic deletions among virusspecific RNA species extracted from persistently infected cells or among viral RNA extracted from concentrated supernatants by RT-PCR methods using different sets of primers to amplify the genomic $L$ and S RNA segments. By using qRT-PCR quantification of the LASV(ML29) NP gene, we demonstrated that S RNA copies rapidly declined during the first five cell culture passages of infected cells and the number of copies persisted at roughly same levels during passages 10-25 (Figure 1b). These levels remain relatively constant for all subsequent passages tested through passage 50 (not shown). In contrast to the permanent level of the S RNA replication and protein expression detected by IF assay (Figure 1D), the infectivity of particles produced by persistently infected cells dropped dramatically during the first 10 passages, was 
below the threshold of detection by passage 15 (Figure 1a), and was not detectable at the final passage 50.

The inability of ML29-persistently infected cells to generate infectious plaques after homologous superinfection with ML29, or infection with LASV/JOS or MOPV, can be partially explained by arenavirus Z-mediated "superinfection exclusion" [249]. However, the replication of the NW mammalian arenavirus TCRV was not affected in ML29/Vero cells (Figure 1f). A recent study documented the capability of LCMV, LASV and MACV mammalian arenaviruses to replicate and disseminate without the Z protein providing evidence that NP can play role as potential surrogate of the Z protein [250]. We assume that ML29 IPs-induced interference was unlikely to be related to Z protein. Nevertheless, the contribution of the Z protein to innate immune responses cannot be fully excluded. ML29 has Z protein derived from MOPV. Interestingly enough, Z protein of pathogenic arenaviruses (including LASV,) but not that of nonpathogenic viruses, including the MOPV Z, inhibit RIG-I-like receptor-dependent IFN type I production [251].

In this study, we failed to provide evidence that ML29 IPs produced by ML29/Vero cells are "classical" defective IPs and the nature of ML29 IP-based interference has to be further elucidated. Nevertheless, ML29 IPs seem to be very attractive for vaccine formulation and development. First, as seen in Figure 2a, ML29P50 generated by ML29/Vero cells, passage 50, were completely attenuated in STAT-1 ${ }^{-/}$mice. These particles were not detectable by plaque assay (test on infectivity) in blood and tissues of mice. However, the level of ML29P50 RNA replication was similar or only slightly reduced in comparison with "wild type" ML29 RNA replication (Figure 2g-i). Second, while ML29P50 and ML29 induced comparable T cell responses at day 7, at the late stage of the infection ML29P50 generated much stronger T cell immunity as assessed by IFN- $\gamma /$ IL-2 ELISPOT in line with the strong immunomodulation potency of defective 
IPs [252]. While mechanism of LASV protection can be dependent on the vaccine platform itself, as well as the animal challenge protocol, in the case of the ML29 vaccine, T cell immunity, but not antibody production, was associated with protection of NHPs [161]. Third, ML29 is the only vaccine with documented evidence of T cell mediated cross protection against Nigerian LASV strains from clade II, among currently available LASV vaccine candidates [125]. This is critical considering the Nigerian LASV strains from clade II are responsible for causing the current unprecedented LF outbreak. LASV genetic diversity is the greatest challenge for vaccine development. The formulation of ML29 with IPs will improve the breadth of the host's immune responses [251] and further contribute to the development of a pan-LASV vaccine with full coverage meeting the WHO requirements [64].

\section{Materials and Methods}

\subsection{Viruses and Cells}

Generation of MOPV An20410, LASV/Josiah, and MOP/LAS reassortant (ML29) LCMV

(WE and ARM) stocks in Vero cells and plaque titration technique was previously described [17]. Freeze-dried Tacaribe virus (TCRV-11573, ATCC ${ }^{\circledR}$ VR-1272CAF ${ }^{\mathrm{TM}}$ ) was purchased from ATCC and the virus stock was prepared by low MOI passage in Vero cells. Infectious plaques for all viruses, except MOPV, were counted at 5 days after infection by treatment of infected cell monolayers under agarose overlay with $4 \%$ paraformaldehyde and staining with $1 \%$ crystal violet. For MOPV, neutral red stain staining was used for plaque development. Vero C1008 cells (Vero 76, clone E6, ATCC $^{\circledR}$ CRL1586) were maintained in DMEM/F-12 supplemented with $10 \%$ fetal calf serum, $1 \mathrm{X}$ antibiotic-antimycotic (ThermoFisher), and 1X Glutamax (ThermoFisher). ML29 persistent infection in Vero cells was established by using a previously described protocol [233, 234]. In brief, after the initial infection of Vero cell monolayers with an MOI of 1.0, at weekly intervals, 
cells were detached from cell tissue flasks by treatment with $0.05 \%$ trypsin-EDTA (ThermoFisher) and sub-cultured at a 1:10 ratio. Culture medium was changed at 3-4-day intervals. Samples of cells and/or culture medium were used for plaque titration, antigen detection assays (IF, Western) and for RNA isolation. To titrate ML29 IPs produced by persistently infected Vero/ML29 cells, monolayers of naïve Vero cells were pre-treated with dilutions of Vero/ML29 passage 5, 20 and 30 supernatants for 1 hour followed by infection with ML29 and detection of plaques as described above. LASV/JOS or EBOV plaque titrations were performed in BSL-4 facilities using maximum containment practices at the University of Texas Medical Branch with the assistance of Dr. Slobodan Paessler's lab.

\subsection{Immunofocus assay}

Vero E6 cells or ML29 persistently infected cells (Vero/ML29) were plated at a density of $2 \times 10^{4}$ cells/well in 96-welltissue culture plates. 24 hours later, cells were overlaid with methylcellulose media. Naïve or Vero/ML29 cells were infected with 0.1 MOI of ML29 for 1 hour prior to being overlaid with methylcellulose. After 3 days, the methylcellulose was removed and cells were then fixed with an acetone methanol mixture. Residual endogenous peroxidase was blocked by hydrogen peroxide and cells were incubated overnight in 10\% FBS. After washing, cells were treated with either polyclonal monkey-anti-ML29 antibody (1:400), monoclonal mouse-anti-LASV/JOS-NP, 1:100 (GenScript, Piscataway, NJ) or polyclonal rabbit-anti-LASV-GP 1:400 (IBT Bioservices, Rockville, MD) followed by HRP linked appropriate secondary antibody. TrueBlue Peroxidase Substrate (SeraCare, Milford, MA) was used for detection. Quantification of staining from high-quality images of scanned plates was performed using Adobe Photoshop to reduce background and threshold images followed by ImageJ counting of pixels.

\subsection{Animal protocols}


4-5 week old female STAT-1 ${ }^{-/-}$mice (129S6/SvEv-Stat1tm1Rds) and wild type controls (129SVE-F) were purchased from Taconic (Hudson, NY). During a 1-week acclimation, a temperature and identification transponder was implanted subcutaneously and the mice were transferred to ABSL-3 housing at the NIH Regional Biocontainment Laboratory on the University of Louisville campus. For infection, $1 \times 10^{3}$ PFU of MOPV, ML29 or ML29P50 (quantitated as a qRT-PCR equivalent dose) was administered (i.p.) in $100 \mu \mathrm{L}$ of PBS. Mice were monitored daily during 21 days and any animal with $25 \%$ weight loss was determined to have met the humane euthanasia criteria. Plasma samples from infected mice that had been euthanized were collected in EDTA tubes (BD). Tissue sample homogenates $(10 \% \mathrm{w} / \mathrm{v})$ were prepared with an Omni TH Tissue Homogenizer in DMEM/F12 followed with centrifugation at 4,500 $\mathrm{g}$ for 20 minutes. Clarified tissue homogenates were tested in the plaque assay described above. All animal protocols were approved by the University of Louisville Institutional Animal Care and Use Committee.

\section{4. $q R T-P C R$ and RNAscope in situ hybridization}

Tissue samples were placed into $2 \mathrm{ml}$ screw-top tubes with $1 \mathrm{ml}$ TRIzol Reagent (ThermoFisher) and glass beads, processed in a bead homogenizer, and tissue homogenates were stored at $-80^{\circ} \mathrm{C}$ until RNA isolation by phenol-chloroform extraction and ethanol precipitation. The qScript cDNA super mix (Quanta Biosciences) was used to make cDNA with an input of 1000 ng of RNA. qRT-PCR with TaqMan primers targeting the MOPV L gene, the LASV NP gene, IL-6, TNF- $\alpha$, or IL-1 $\beta$ were used with a $18 \mathrm{~S}$ housekeeping probe on a StepOnePlus qRTPCR analyzer (Applied Biosystems). All IL-6, TNF- $\alpha$, IL-1 $\beta$ and 18S primer/probe sets were commercially purchased through ThermoFisher Scientific through the Applied Biosystems division. Primer/probe set for MOPV amplified a region of the L segment (FWD 5'TCCTCAATTAGG CGTGTGAA-3'; REV 5'-TACACATCCTTGGGTCCTGA-3'; probe 6FAM- 
CCCTGTTCCCTCCAACTTGTTCTT TG-TAMRA). Primer/probe set for LASV (ML29) amplified the NP gene segment (FWD 5'-TCC AACATATTGCCACCATC-3'; REV 5'- GCT GAC TCA AAG TCA TCC CA-3'; probe 6FAM TGCCTTCACAGCTGCACCCA-TAMRA). The qRT-PCR reaction contained $5 \mu \mathrm{M}$ of each primer and $2 \mu \mathrm{M}$ of probe for each primer/probe set, $5 \mu \mathrm{l}$ of cDNA and TaqMan Fast Advanced Master Mix (ThermoFisher) in a final reaction volume of $20 \mu \mathrm{L}$. The reaction conditions were as follows: $50^{\circ} \mathrm{C}$ for $2 \mathrm{~min}, 95^{\circ} \mathrm{C}$ for $20 \mathrm{sec}$ then 40 cycles alternating between $95^{\circ} \mathrm{C}$ for $3 \mathrm{sec}$ and $60^{\circ} \mathrm{C}$ for $30 \mathrm{sec}$. For RNAscope hybridization, fixed tissue sections were embedding in paraffin, cut on a Leica RM2125 RTS microtome, and mounted on glass slides. Custom designed target probes, preamplifier, amplifier, and label probe targeting MOPV L RNA were synthesized by Adanced Cell Diagnostics (Hayward, CA) and RNAscope assay was performed according to the manufacturer's manual. Chromogenic detection was performed using DAB followed by counterstaining with hematoxylin.

\subsection{EISPOT and IgG ELISA}

Assessment of T cell responses in infected mice by the murine IFN- $y /$ IL-2 Double-Color Enzymatic ELISPOT (Cellular Technology Ltd., Cleveland, OH) has been recently described [236]. In brief, erythrocyte-free splenocytes were added to 96-well filter plates (Millipore, MSIPS4510) pre-coated with anti-mouse cytokine antibody in triplicate at a density of $4 \times 10^{5}$ cells/well. Cells were stimulated overnight at $37^{\circ} \mathrm{C}$ with cocktails of $10 \mu \mathrm{M}$ LASV/JOS or MOPV GPC peptides (Mimotopes Ltd, Melbourne, Australia). Each cocktail contained 69 overlapping 20-mer peptides. As positive and negative controls, Conconavalin A (ThermoFisher) and CLT media alone was added to quality control wells. After stimulation, plates were developed according to the manufacturer's protocol and cells secreting individual cytokines IFN- $\gamma$ or IL-2 or both were counted using C.T.L. Ltd. Immunospot ${ }^{\circledR}$ S5 Micro-analyzer and Immunospot ${ }^{\circledR}$ V 4.0 software. Quality control analysis was provided by C.T.L. Ltd. 
IgG ELISA was performed as previously described [165]. In brief, microtiter plates were coated with $5 \times 10^{6} \mathrm{PFU} /$ well of sonicated virus suspension in carbonate buffer (Sigma) overnight. Plates were washed, blocked with $10 \%$ milk for 2 hours, washed again, and coated with dilutions of mouse serum for 1 hour. Plates were washed and secondary HRP linked rabbit-anti-mouse IgG (Sigma) was added at a 1:2,500 dilution for 1 hour. Plates were washed and SureBlue TMB peroxidase substrate (SeraCare) was added for 15 minutes before the addition of TMB Stop Solution (SeraCare).

\subsection{Statistics Analysis}

Results are reported as means \pm SEM $(n=4-5)$. ANOVA with Bonferroni's post hoc test (for parametric data) or Mann-Whitney Rank Sum test (for nonparametric data) was used for the determination of statistical significance among treatment groups, as appropriate. Statistical analysis (mean, SD, T-test) and graphics were performed using the GraphPad Prism version 7 for Windows package (GraphPad Software, LaJolla, CA).

Author Contributions: Conceptualization, ISL; Methodology, ISL, DMJ, JDJ; Animal Studies, DMJ, JDJ; Data Analysis, ISL, DMJ, JDJ; Visualization, DMJ, JDJ; Writing, ISL, DMJ; Funding Acquisition, ISL; Supervision, ISL.

Funding: This work was supported by grants R01AI093450 and R56Al135770 from the National Institute of Allergy and Infectious Diseases.

Acknowledgments: We thank the University of Louisville Center for Predictive Medicine for Biodefense and Emerging Infectious Diseases for the support of animal studies as well as Dr. Slobodan Paessler (The University of Texas Medical Branch at Galveston) for titration of Lassa and Ebola samples using Vero/ML29 cells. 
Conflicts of Interest: The authors declare no conflict of interest.

Figures

(a)

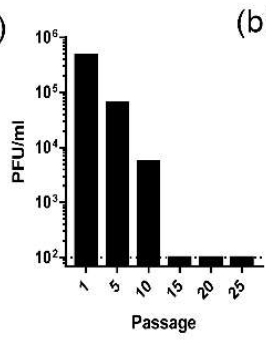

(b)

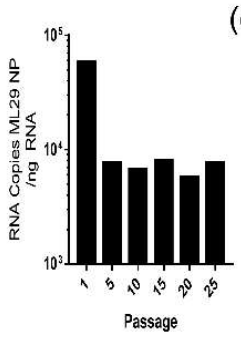

(c)

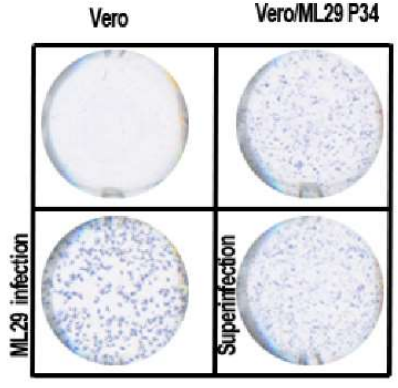

LCMV/ARM

(e)
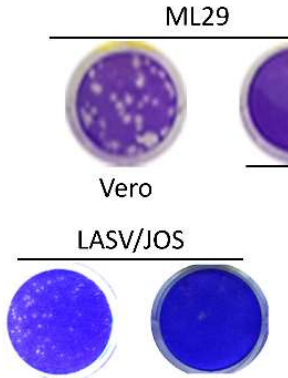

Fig. 1

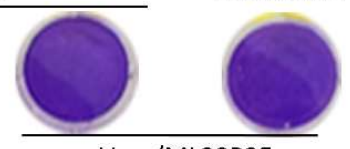

Vero/ML29P25

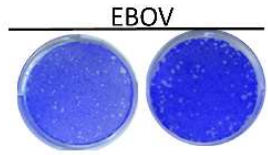

Vero Vero/ML29P25 (d)

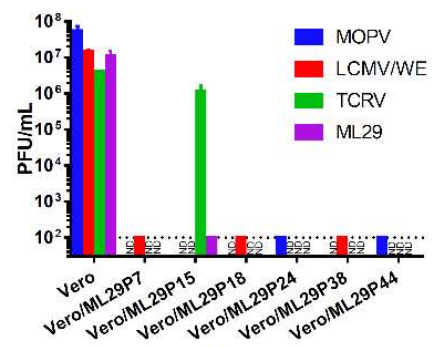

Cells Infected

(f)

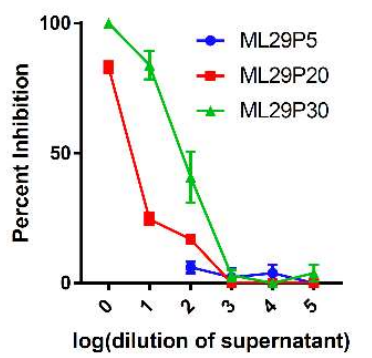

Figure 1. Vero cells persistently infected with ML29 interfere with replication of homologous and genetically-related arenaviruses. (a) Supernatants from a Vero cell line persistently infected with ML29 (Vero/ML29) were titrated for infectious virus by plaque assay at the indicated passages. (b) Total RNA was isolated from a Vero/ML29 cells at the indicated passages and was assayed using qRT-PCR with a LASV/JOS NP TaqMan probe. Copy number was determined by regression against a standard curve. (c) Immunofocus assay using polyclonal monkey-anti-ML29 on uninfected Vero E6 cells (top left), Vero/ML29 passage 34 cells (top right), Vero E6 cells infected with a $0.1 \mathrm{MOI}$ of ML29 for 3 days (bottom left), and Vero/ML29 passage 34 cells 
superinfected with a $0.1 \mathrm{MOI}$ of ML29 for 3 days (bottom right). (d) Naïve or persistently infected Vero/ML29 cells at different passages were used to titer MOPV, LCMV/WE, TCRV, ML29 by plaque assay. (e) Plaque-forming activity of Vero/ML29 cells. ML29 (top left and top center), LCMV/ARM (top right), LAS/JOS (bottom left and bottom left center), and EBOV (bottom right center and bottom right) were used in a plaque assay on Vero E6 cell (top left, bottom left, and bottom right center) or the 25th passage of a Vero cell line persistently infected with ML29, Vero/ML29P25 (top center, top right, bottom center left, bottom right.) (f) Dose-dependent effects of IPs on replication of ML29. Vero E6 cells were pre-incubated for 1 hour with the indicated dilution of ML29 IPs produced by Vero/ML29 cells at passage 5, 20 or 30 . The pretreated cells were then infected with standard ML29 and viral titer was determined by plaque assay. Percent inhibition is normalized to infectious titer determined on Vero E6 cells that were not pretreated.

(a)

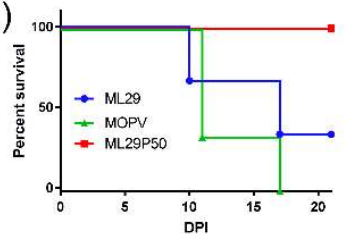

(d)

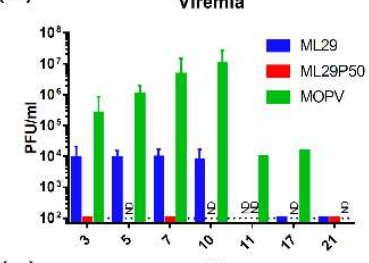

(g)

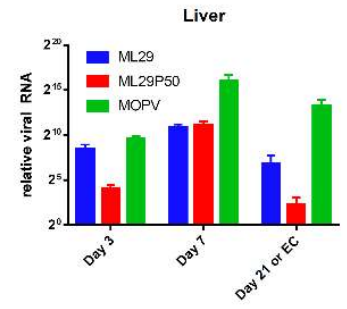

(b)

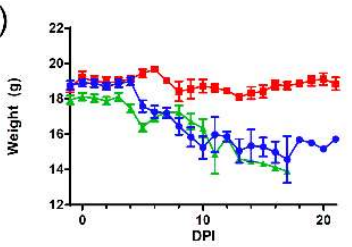

(e)

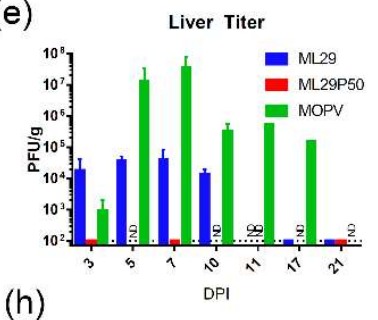

(h)

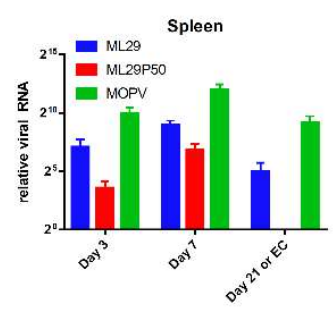

(c)

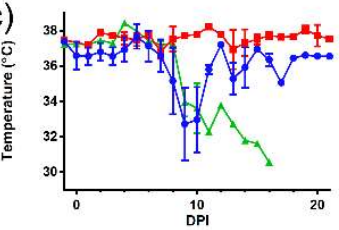

(f)

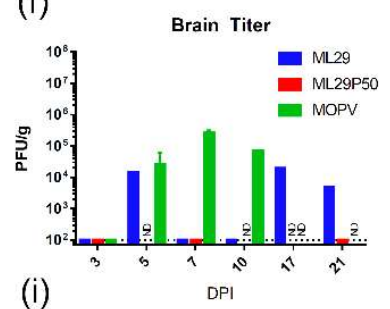

(i)

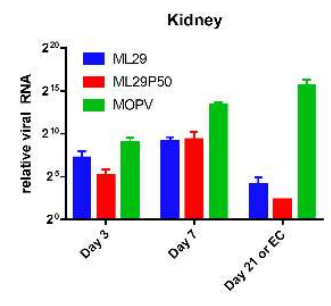


Figure 2. Clinical manifestation and outcome of MOPV and ML29 infections in STAT-1 ${ }^{-1}$ mice. (a) Mice were infected (i.p.) with $1 \times 10^{3}$ PFU of MOPV An20410, ML29 (standard infectious virus) or with ML29P50 (IPs collected from culture medium of Vero/ML29P50 cells). ML29P50 IPs were quantified by MOPV L gene qRT-PCR and used in genome-equivalent dose, $1 \times 10^{3}$ PFU. (b) Weight and (c) Temperature of infected mice. (d) Viremia, (e) Infectious viral load in liver, and (f) brain of STAT-1 ${ }^{--}$mice. (g-i) Viral RNA load assessed as relative expression of MOPV L gene by qRT-PCR in the indicated tissues at the time point indicated. DPI, days post infection. EC, endpoint criteria. 


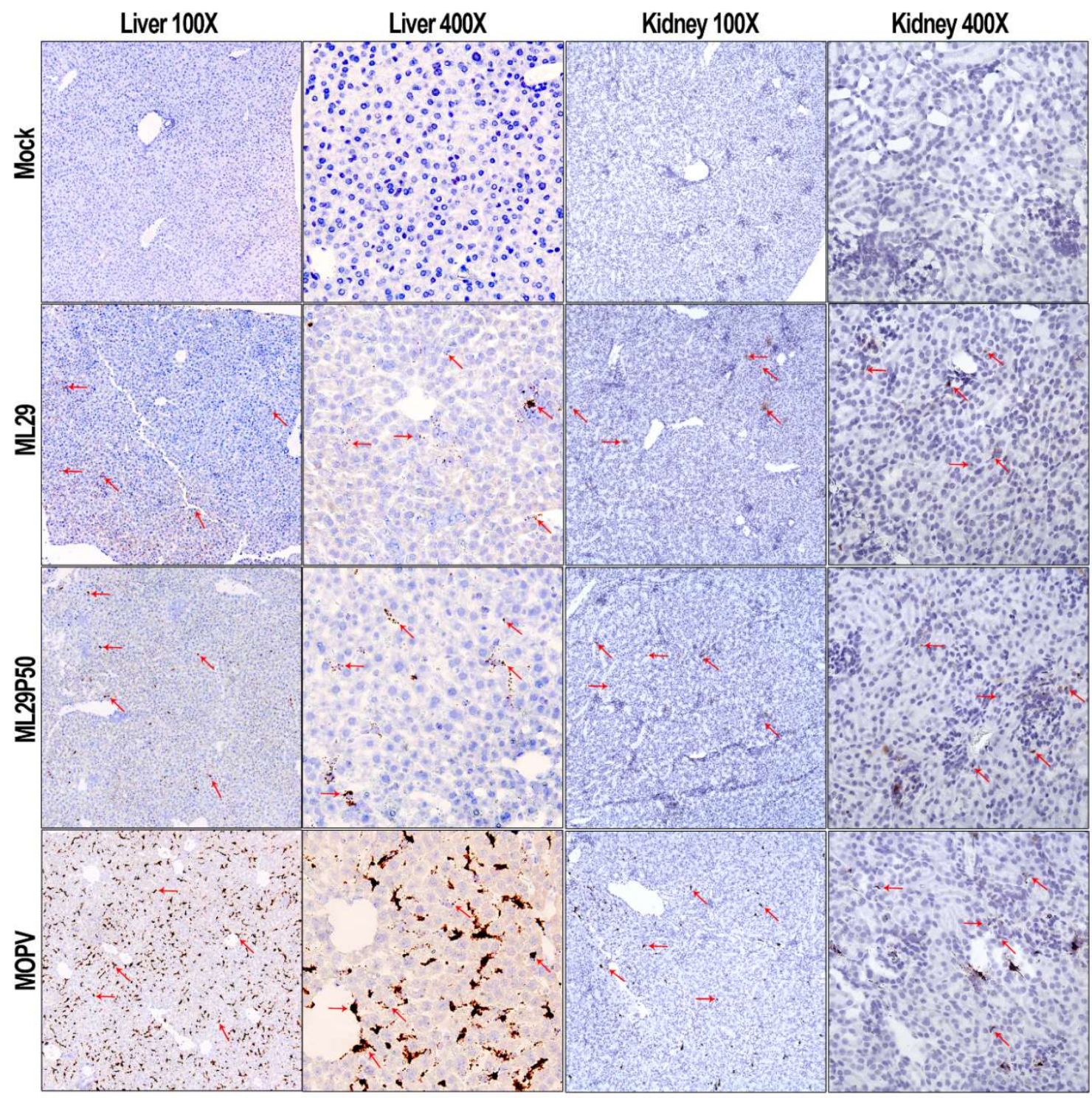

Figure 3. RNAscope in situ hybridization. Representative images at two different magnifications (100x and 400x) of RNAscope in situ hybridization (brown spots, arrowed) targeting the MOPV L gene in the liver and kidney of STAT1 $\%$ mice. 


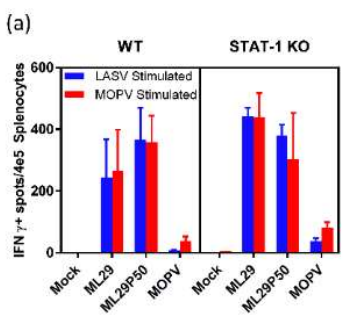

(d)

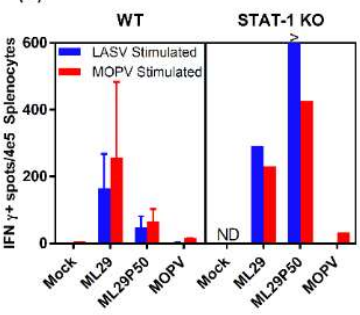

(b)

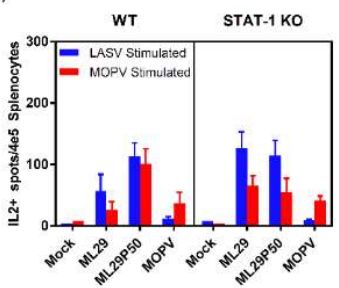

(e)

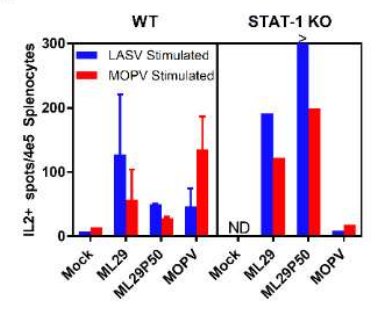

(c)

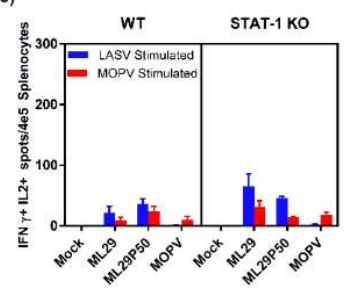

(f)

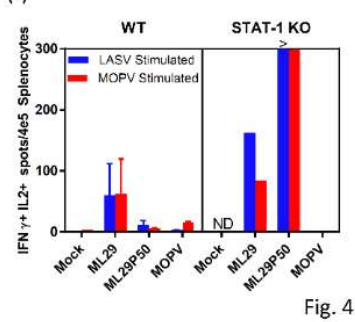

ELISA

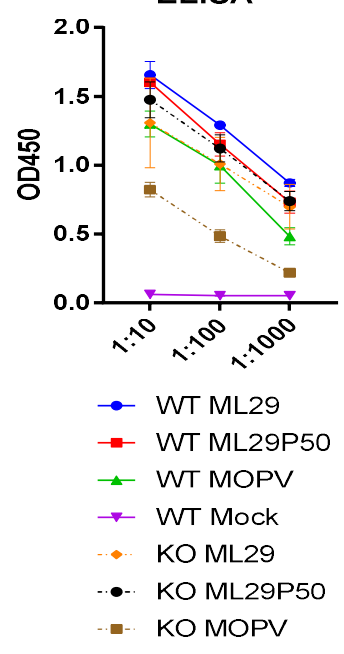

Figure 4. Adaptive immune responses of STAT-1 ${ }^{\%}$ mice. ELISPOT counts per $4 \times 10^{5}$ splenocytes from STAT-1 ${ }^{--}$mice or wild type (WT) controls collected (a-c) 7 days post infection or (d-f) at endpoint criteria or 21 days post infection. Splenocytes were stimulated with LASV GPC or MOPV GPC-derived peptide cocktails for 24 hours. (a, d) IFN- $\gamma$-secreted cells, (b, e) IL-2-secreted cells, or (c, d) poly-functional IFN- $/$ /IL-2-secreting cells. (g) Antibody responses measured by IgG ELISA in the serum of STAT-1\% mice or wild type controls collected at 21 days post infection or endpoint criteria. $>$, Too numerous to count.

(a)

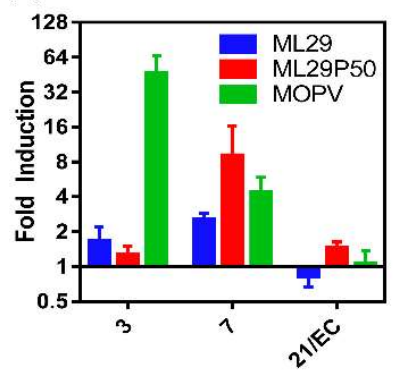

(b)

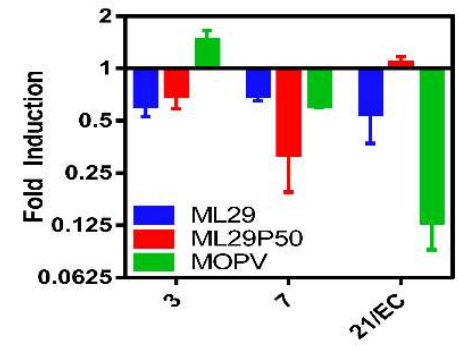

(c)

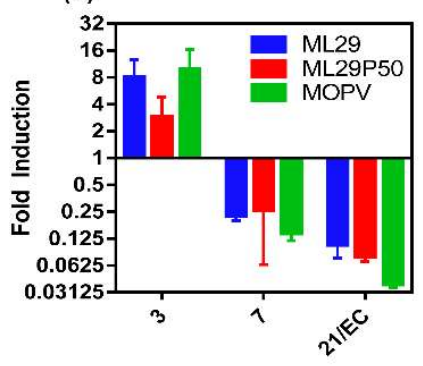

Figure 5. Cytokine responses of STAT-1 ${ }^{-1-}$ mice. (a) IL-6, (b) TNF- $\alpha$, or (c) IL-1 $\beta$ expression in splenocytes of STAT-1 ${ }^{-1-}$ mice relative to mock infected by qRT-PCR. 

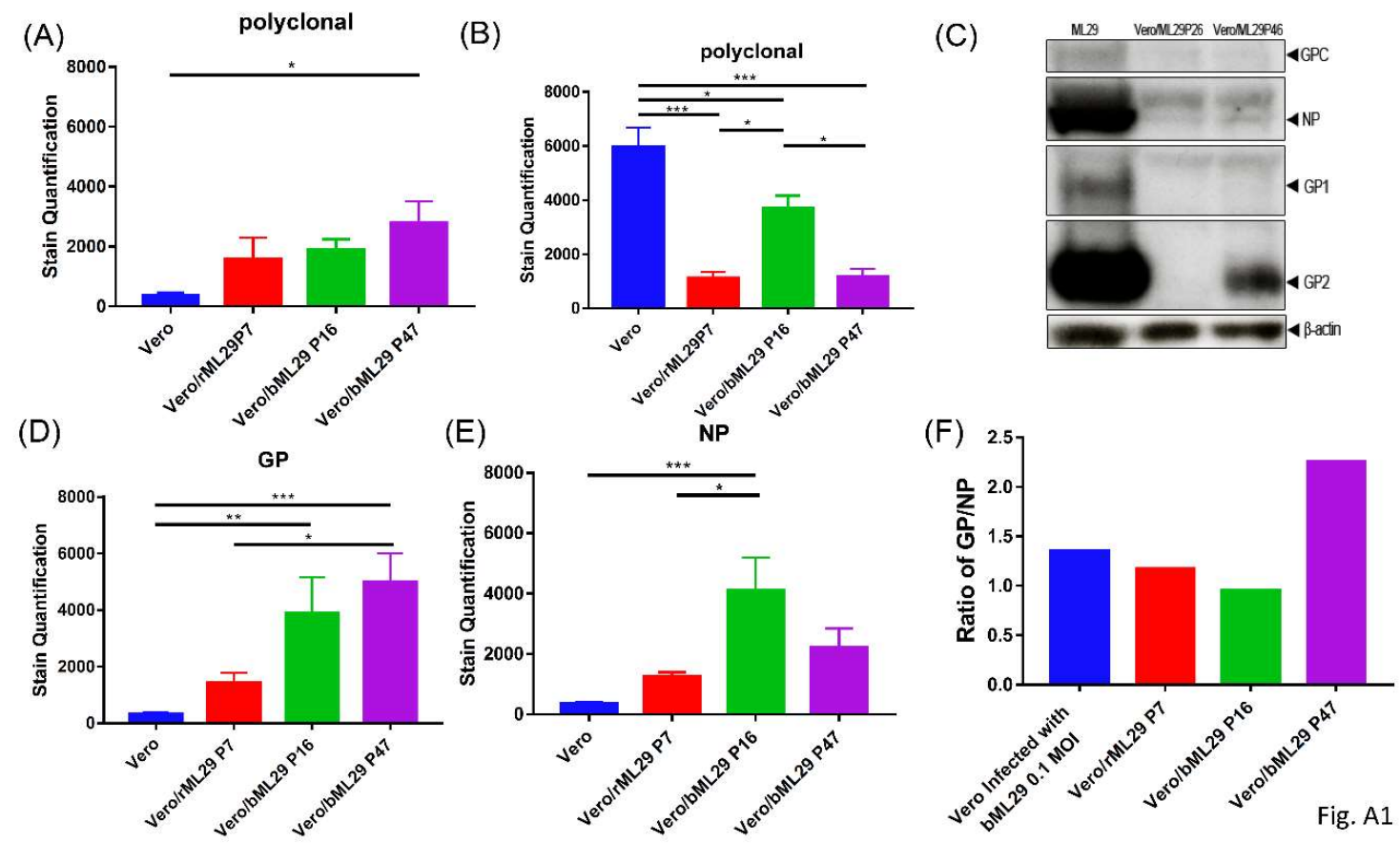

Figure 6. Quantification of immunofocus staining using polyclonal moneky-anti-ML29 on the indicated cell lines (A) without additional treatment, or (B) 3 days following infection with a 0.1 MOI of ML29. (C) Western blot of cell lysates from ML29, Vero/ML29P26, and Vero/ML29P46 detected with polyclonal monkey-anti-ML29 antibody. (D-E) Quantification of immunofocus staining using (D) polyclonal rabbit-anti-LASV-GP 1:400 or (E) mouse-anti-LASV/Jos-NP 1:100 antibodies. (F) The calculated ratio between staining quantified in (D) and (E). ${ }^{*} p<0.05,{ }^{* *} p<$ $0.01, * * * p<0.001$. 
(A)

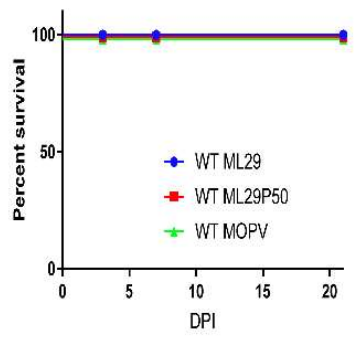

(D)

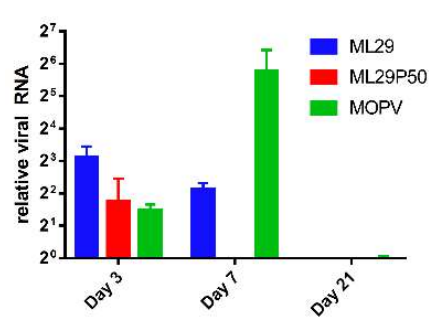

(B)

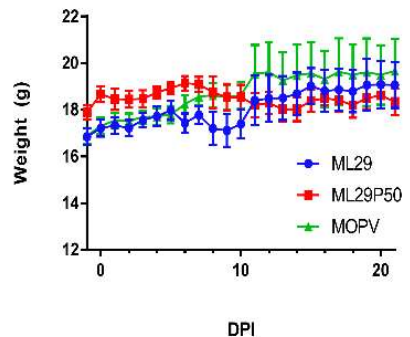

$(\mathrm{E})$

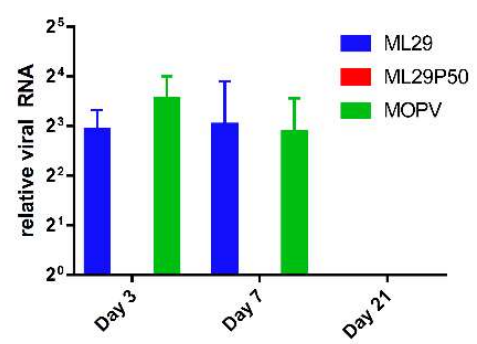

(C)

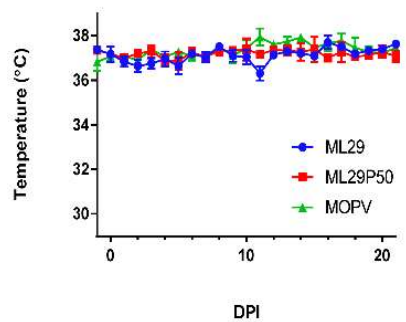

$(\mathrm{F})$

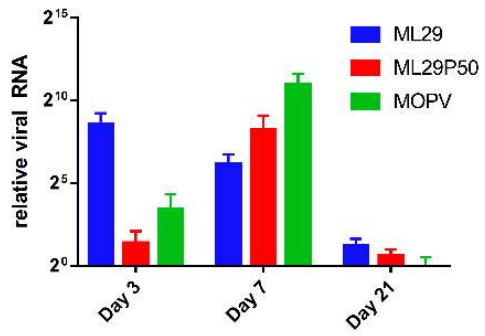

Figure 7. (A) Survival of wild type 12956 mice infected IP with $1 \times 10^{3}$ PFU of ML29, $1 \times 10^{3}$ PFU of MOPV, or a MOPV L gene qRT-PCR equivalent dose of supernatant from passage 50 Vero/ML29 cells. Daily (B) weight and (C) temperature of mice from (A). (D-F) Relative expression of MOPV L gene by qRT-PCR in the (D) liver, (E) spleen, and (F) kidney of wild type $129 \mathrm{~S}$ at the time points indicated post infection.

(A)

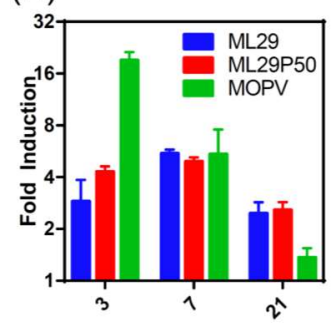

(B)

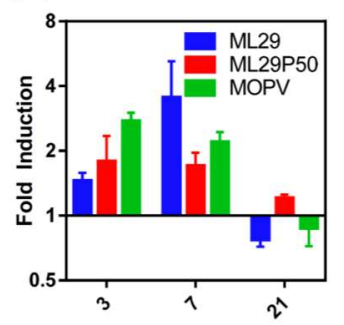

(C)

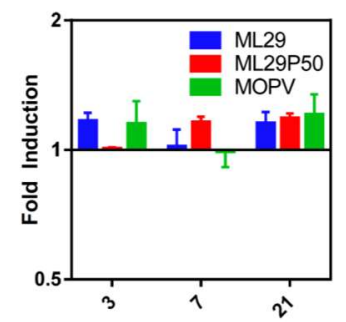

(D)

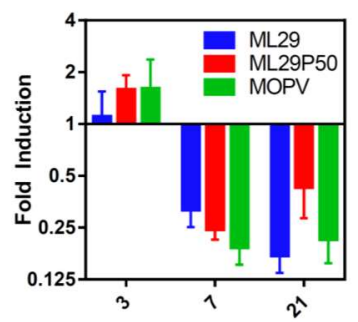

Figure 8. qRT-PCR analysis showing IL-6 expression in the (A) liver and (B) spleen , (C) TNF- $\alpha$ in the spleen, or (D) IL-1ß expression in the spleen of wild type 12956 mice infected with the indicated viruses relative to mock infected at days 3,7 , and 21 post-infection.

\section{Supplemental Materials and Methods}


Quantification of immunofocus staining from high-quality images of scanned plates was performed using Adobe Photoshop to reduce background and threshold images followed by ImageJ counting of pixels. Statistical significance was tested with a one-way ANOVA using GraphPad Prism version 7.

For western blotting, a T-75 cell culture flask with a confluent monolayer of either Vero cells was infected with a $1 \mathrm{MOI}$ of ML29 5 days prior, or Vero/ML29, was harvested in Tris-buffer containing 1.0\% NP-40, protease inhibitors and 0.1\% SDS. Protein content was standardized based on a pierce modified Lowry protein assay (ThermoFisher) denatured by boiling in Laemmli's buffer and run on a 10\% acrylamide gel at $120 \mathrm{~V}$ for 1 hour. The proteins were transferred to a nitrocellulose membrane, blocked with $5 \%$ milk, and detected with a 1:1000 dilution of polyclonal monkey-anti-ML29 antibody. 


\section{CHAPTER IV}

\section{SAFETY AND IMMUNOGENICITY OF A DEFECTIVE INTERFERING PARTICLE ENHANCED LASSA VIRUS VACCINE CANDIDATE IN STAT-1-/- MICE, CBA MICE, AND GUINEA PIGS}

\section{Introduction}

Defective interfering particle enhanced ML29 (ML29P50) is attenuated in STAT-1\% mice compared to standard ML29 preparations [204]. Additionally, ML29P50 elicits greater specific Tcell mediated immunity than ML29 upon stimulation with both homologous peptides from Lassa virus (LASV) strain Josiah (LASV/Jos) glycoprotein precursor (GPC) and heterologous peptides from Mopeia virus (MOPV) [204].

Retinoic acid-inducible gene I (RIG-I), melanoma differentiation-associated antigen 5 (MDA-5), and dsRNA-dependent protein kinase $\mathrm{R}$ (PKR) are intracellular receptors that recognize arenavirus RNA during replication and induce innate immunity through activation of Nuclear Factor Kappa B (NF-KB), Interferon Response Factor 3 (IRF-3), and Interferon Response Factor 7 (IRF-7) [253-255]. Old world arenaviruses including LASV, MOPV, and Lymphocytic Choriomeningitis virus (LCMV) antagonize these responses through the exonuclease activity of viral nucleoprotein (NP), and by the binding of the matrix RING-finger protein (Z) to the cellular RNA receptors [256, 257]. IRF-3 and IRF-7 play an important role in generating interferon responses and apoptosis in a STAT-1 dependent manner [258, 259]. IRF-3 also stimulates chemokine and cytokine production in a STAT-1 independent fashion [259]. NF-kB activation can stimulate pro-inflammatory cytokine signaling and is important in the induction of adaptive immunity [260]. However, it has been reported that even in the absence of IFN signaling, MOPV, and to a lesser extent LASV, are able to induce interferon stimulated genes (ISGs) [254], potentially through the actions of NF-KB and IRF-3. Notably, the NP-inducible anti-IFN activities 
of arenaviruses are common features of mammalian arenaviruses regardless of their pathogenic potential for humans [256, 261, 262].

Defective interfering genomes (DIG) have been described for most RNA viruses [263]. Classically, DIGs contain large deletions while preserving sequences required for replication and packaging into virions to create defective interfering particles (DIPs) [263]. Because DIGs are shorter than full length viral genomes, it is thought that they more efficiently compete for replicative machinery, which is responsible for the observed interference [263]. However, other mechanisms of DIG interference, including modulation of antiviral apoptosis responses, has also been observed [264]. For many viruses, DIPs can regulate virulence while stimulating host immunity [263]. A key component of this regulation is the induction of interferon responses and pro-inflammatory cytokines by DIPs [252, 265-268]. The inflammatory regulation for many DIPs can be attributed to engagement of RIG-I and MDA-5 [269, 270]. Additionally, DIPs may play a role in enhancing antigen presentation [271], which makes them an attractive tool for vaccine enhancement. A vaccine enhanced with DIPs would presumably have enhanced capacity for induction of innate immunity and superior antigen presentation. Additionally, it has been speculated that the interfering activity of DIPs could be exploited as antivirals [272]. The antiviral activity of DIPs may enhance the post-exposure prophylaxis therapeutic window for vaccination.

DIP production by LCMV is thought to play a key role in the establishment of persistence in the rodent host $[232,273]$. In LCMV, it has also been shown that phosphorylation of the lategene motif PPXY may play a role in the regulation of DIP production [273]. A key difference in DIPs observed from ML29 compared to those of LCMV, is that LCMV DIPs tend to exert waxing and waning interference (observed by increases and decreases in the infectious particle production from chronically infected cells) [274], while ML29 DIPs are associated with complete 
abrogation of infectious particle production shortly after establish of persistent infection (15 passages of ML29-infected cells) [204].

The genetic diversity of LASV has been one of the greatest challenges to vaccine development [67]. Currently, LASV strains are split into 4-6 phylogenetic lineages, which circulate in Nigeria and different areas of West Africa [275]. Interference mediated by DIPs provides a very attractive approach to overcome these phylogenetic barriers and develop panLASV preventive and therapeutic strategies.

DIGs still require wild-type (full-length) viral genomes for replication, which complicates detection of DIGs. If the DIGs were truncations instead of internal deletions, conventional PCR or sequencing methods would not be useful for detecting them. The presence of wild-type genomes in DIP can mask the presence of ML29 DIGs. For the New World arenavirus Machupo (MACV), deletions were detected by analysis of chimeric reads from next generation sequencing of viral stocks with reduced pathogenicity [276]. However, the direct contribution of these chimeric sequences to the interference of pathogenicity is unknown.

In our study, Northern blotting (NB) was used to detect truncated viral RNAs directly from ML29-persistently infected cell cultures. Additionally, a minigenome-like L-segment with significant or total deletion of the L-gene was used to test the hypothesis that synthetic DIPs, similar to those noted for influenza [277], could interfere with arenavirus replication.

Another challenge to address concerning LASV vaccine development is the potential for adverse drug effects including sensorineural hearing loss. LASV associated sensorineural hearing loss, thought to be due to T-cell mediated autoimmunity during convalescence, is a leading cause of deafness in West Africa [278-280]. To some extent, the STAT-1 ${ }^{--}$mouse model recapitulates the hearing loss observed following LASV infection in humans and was proposed as 
a model to test hearing loss as a possible adverse reaction following vaccination [216]. We hypothesized that because ML29 is able to induce robust T-cell responses in STAT- $1^{-1-}$ mice [204], that if these T-cell responses cause cochlear damage following ML29 vaccination, it would cause detectable hearing loss in the mice. Additionally, since T-cell responses were stronger in ML29P50 vaccinated mice [204], these mice would seem to be even more susceptible to postvaccination hearing loss.

In this study, the highly susceptible CBA/J model, and a Hartley guinea pig model that more closely mimics human responses to LASV infection were used further assess the safety of ML29 and ML29P50. CBA/J mice intracranially (IC) infected with ML29 or ML29P50 were used to confirm the attenuated phenotype of ML29P50, [17, 165]. Hartley outbred guinea pigs (Cavia porcellus) are susceptible to infection with some strains from LASV clade IV (LF2384 strain without adaptation [281] and LASV/Jos with adaptation [282],) and Nigerian LASV strain from LASV clade III [127]. Additionally, while strain 13 guinea pigs infected with LASV tend to show signs of airway disease, LASV infection of Hartley guinea pigs causes disease more typical of that observed in humans $[123,282]$. Hartley guinea pigs were used to screen for signs of viral hepatitis and renal disease following vaccination with ML29 and ML29P50. Additionally, this model was used to investigate the potential for heterologous protection from LCMV strain WE (LCMV-WE) following ML29 vaccination.

\section{Methods}

Establishment of Vero cells persistently infected with ML29 and assessment of heterologous interference of persistently infected cells

A persistently-infected subline of Vero cells was established as previously described [204]. In brief, Vero cells were initially infected with ML29 at a 0.01 multiplicity of infection 
(MOI), and subjected to serial passages passaged normally every 7 to 10 days. Cell culture medium (ThermoFisher \#11330032 supplemented with 1X antibiotic antimycotic \#15240062, 1Xglutamax \#35050061, and 10\% FBS \#10438026,) was removed and cell monolayers were washed with PBS. Cells were detached from tissue flasks with a short treatment (5-10 minutes) with $0.05 \%$ Trypsin-EDTA (ThermoFisher \#25300120), suspended in fresh culture medium, counted and plated in new tissue flasks at a 1:10 to 1:5 ratio as previously described [204]. A naming scheme of "VeroML29PX" was employed to refer to the passaged cells with " $X$ " being replaced with the passage number. VeroML29P25 and VeroML29P49 were cryopreserved at a density of $1 \times 10^{7}$ viable cells per $1 \mathrm{ml}$ of cell culture media containing 5\% DMSO (VWR \#97063136). Cells were recovered and passaged several times before VeroML29P28 and VeroML29P53 were plated at a density of $4 \times 10^{5}$ cells per well in 6-well tissue culture treated plates. After passage 15 , persistently-infected cells failed to generate infectious "plaques" after infection with ML29 and the genetically-related viruses, LASV and LCMV [204]. To further assess the mechanisms of heterologous interference, VeroML28P28 and -P53 cells were infected with $3.4 \times 10^{4}$ foci forming units (FFU) of tri-segmented Lymphocytic Choriomeningitis Virus strain Armstrong (LCMV-Arm) expressing green fluorescent protein (GFP) from both the nucleoprotein (NP) and glycoprotein precursor (GPC) loci from alternate S-segments, r3LCMV-Arm-GFP [283]. At 24 and 48 hours post-infection (HPI) images were captured with a BZ-X800 fluorescent microscope (Keyence, Itasca, IL, USA) with a $488 \mathrm{~nm}$ filter using $40 \%$ excitation light, $1 / 8.5 \mathrm{~s}$ exposure, and a $4 \mathrm{X}$ lens in high sensitivity mode from the center of the well. The images were then compiled in Adobe Photoshop (Adobe Inc., San Jose, CA, USA) and the brightness and contrast were uniformly enhanced for visualization (brightness setting 56 , contrast 88. )

\section{Northern Blotting}


Vero cells were plated on 6 well tissue culture plates at a density of $4 \times 10^{5}$ cells per well and allowed to sit overnight prior to infection with $0.001 \mathrm{MOI}$ of ML29, LASV/Jos (in the BSL-4 facility at UTMB, Galveston), or MOPV strain AN20410 in $200 \mu \mathrm{L}$ of $2 \%$ FBS cell culture media. Infection was allowed to occur on a rocking plate in a $37^{\circ} \mathrm{C}, 5 \% \mathrm{CO}_{2}$ incubator. After infection, the virus inoculum was aspirated and replaced with $2 \mathrm{ml}$ of $2 \% \mathrm{FBS}$ cell culture medium. At the indicated times, the cell supernatants were aspirated, and $1 \mathrm{ml}$ of TRIzol (ThermoFisher \#15596018) was pipetted over the cells 5-10 times before being transferred to a microfuge tube. Wells harvested before the end of the experiment were washed three times with $10 \%$ FBS in PBS to quench the phenol. RNA was isolated from the TRIzol with 1-bromo-3-chloropropane (Millipore Sigma \#B9673) according to the manufacturer's directions and stored in $30 \mu \mathrm{l}$ of FORMAzol (Molecular Research Center, Inc., \#FO 121).

A $1.2 \%$ agarose gel containing $1 X$ MOPS buffer and $6.6 \%(\mathrm{w} / \mathrm{v})$ formaldehyde was made using DEPC water. Five $\mu \mathrm{g}$ of each RNA sample was mixed with $3.5 \mu$ of $37 \%(w / v)$ formalin, $2 \mu l$ 10X MOPS buffer, and $2 \mu \mathrm{g}$ of ethidium bromide in a total volume of $20 \mu \mathrm{l} \mathrm{FORMAzol} \mathrm{prior} \mathrm{to}$ incubation for 15 minutes at $65^{\circ} \mathrm{C}$. After rapid cooling, samples were mixed with loading buffer and run for 3 hours at $80 \mathrm{~V}$. The gel was then washed with two changes of DEPC water, followed by $10 \mathrm{mM}$ sodium phosphate for 10 minutes each on an orbital shaker. The gel was then blotted onto a nylon membrane by upward capillary transfer, and UV cross-linked. Templates for probe generation were PCR amplified from ML29 L segment (for ML29 and MOPV detection) using forward (GATGT CAACA GATGA TAAGT CAGGC TTTAA AG) and reverse (CACCG GGGAT CCTAG GCATT G) primers, ML29 S segment (for ML29 detection) with forward (GGCCT TTCTG TTCTG ATCAC CTTTG A) and reverse (CGCAC AGTGG ATCCT AGGCT ATTGG) primers, or LASV L segment with forward (TCAGG GACTG TAGGG TGGGG GT) and reverse (ATGGG AAACA AGCAA GCCAA AGC) primers. Phosophorus-32 labeled ribonucleotides were used to generate probes from the 
PCR amplified DNA, and the radioactive probes were hybridized to the nylon membrane. The membrane was washed, and exposed to film for 6 hours to develop.

Quantification of virus stocks from the supernatant of VeroML29P50 cells

Supernatants from VeroML29P50, referred to here as ML29P50, were quantified based on quantitative reverse transcriptase PCR (qRT-PCR) as described previously [204]. Briefly, RNA from ML29 and ML29P50 viral stocks was extracted in TRIzol LS (ThermoFisher \#1026028) and reverse transcribed with qScript cDNA SuperMix (QuantaBio \#95048, Beverly, MA, USA), prior to qPCR on a StepOnePlus analyzer (Applied Biosystems) using TaqMan Fast Advanced Master Mix (Thermo Fisher \#4444965). Custom primers specific for the ML29 NP gene (FWD 5'-TCCAA CATAT TGCCA CCATC-3'; REV 5'- GCTGA CTCAA AGTCA TCCCA-3'; probe 6FAM TGCCT TCACA GCTGC ACCCA -TAMRA (ThermoFisher) along with a Eukaryotic 18S rRNA endogenous control (VIC/TAMARA) (ThermoFisher \#431093E) were used. Dilutions of ML29 were used to make a standard curve based on the assumption that one copy of viral RNA detected correlates with one PFU of infectious virus. The number of ML29P50 copies per volume were plotted on this curve to determine the equivalent concentration of virus in the stock.

Transfection of cells with minigenome (MG) constructs derived from the LCMV L RNA

Arenavirus MGs provide valuable tools to study mechanisms of replication and transcription in vitro [181]. Most arenavirus MG systems are based on the S RNA sequences, and include minimal cis- (5' and $3^{\prime}$ untranslated regions [UTRs] and the intergenic region [IGR]), and trans- (NP and L protein) elements required for replication/transcription. To study defective genomes derived from arenavirus L RNA, we designed MG system based on LCMV L RNA. In brief, the full length L-Segment of LCMV-Arm was replaced with a GFP gene, and named LCMV\#1074. A similar construct was also made, except 198 nucleotides of the $\mathrm{N}$-terminus 
coding region of $L$ was left intact, followed by a P2A linker, and a gene encoding for the fluorescent reporter ZsGreen, called LCMV\#1076. These constructs were tested in the previously described LCMV rescue system [284, 285]. Briefly, BHK-21 cells were plated in 6 well tissue culture treated plates and allowed to rest for one day, at which point they were about $85 \%$ confluent. They were then transfected with the LCMV\#1074 or LCMV\#1076 constructs, using the Lipofectamine 2000 system (ThermoFisher \#11668030) along with pCAGGs expression vectors coding for the LCMV L-RdRP protein and the LCMV nucleoprotein NP $[193,286]$. In our experiments, BHK-21 cells were also transfected with LCMV\#1074 or LCMV\#1076 as described above, but without the trans-acting factors required for rescue. These cells were then infected with a 0.1 or $0.5 \mathrm{MOI}$ of LCMV-Arm one-day post transfection to provide "helper" virus for $L$ and NP production. The supernatant of these cultures was collected two days following transfection and titered on BHK-21 cells with detection of focus forming units (FFU) using anti-LCMV NP antibody VL-4 (BioXcell West Lebanon, NH, USA) conjugated to a $488 \mathrm{~nm}$ fluorescent dye using an antibody labeling kit (ThermoFisher \#53024).

In vivo $C B A / J$ and STAT-1\% mouse experiments

Four week old female CBA/J were purchased from The Jackson Laboratory (Bar Harbor, ME, USA). Fifteen mice were randomly assigned to each group $(n=15)$ and allowed to acclimate for one week. Following acclimation, mice were inoculated IC with $10^{3}$ PFU of ML29, the qRTPCR equivalent dose of ML29P50 (approximately a 1 to 20 dilution of the stock), or sterile PBS for mock treatment in a total volume of $10 \mu$ l. Mice were observed daily for up to two weeks for survival, weight, and clinical score based on a composite of grooming, adjusted grimace scale, activity and neurological manifestations, as previously described [44]. 
Four-to-five week-old female STAT-1/- mice (129S6/SvEv-Stat1tm1Rds) were ordered from Taconic (Hudson, NY, USA). During a 1-week acclimation, mice were randomized into groups of 12 for ML29 and MOPV or 6 for ML29P50 or mock treatment. Prior to treatment, the mice were transferred to ABSL-3 housing at the NIH Regional Biocontainment Laboratory on the University of Louisville campus. At day 0 , mice were inoculated via the intraperitoneal route (IP) with $10^{3}$ PFU of ML29, the qRT-PCR equivalent dose of ML29P50 (approximately a 1 to 20 dilution of the stock), MOPV, or sterile PBS for mock in a total volume of $100 \mu$ l. Mice were observed daily for up to 62 days for survival, weight, and clinical score. Hearing tests were conducted by acoustic startle with a SR-Lab startle response system (San Diego Instruments, San Diego, CA, USA) as previously described [216]. Supportive care of $1 \mathrm{ml}$ of warmed $0.9 \% \mathrm{NaCl}$ given subcutaneously (SC), access to sterile water soaked mouse chow on the cage floor, and DietGel 76A (Clear H2O, Portland, ME, USA) were provided to all mice in the study during 7-12 DPI. After 12 DPI, supportive measures were discontinued for mock and ML29P50 groups based on veterinary advice, while mice in ML29 and MOPV groups received either $1 \mathrm{ml}$ (13-15 DPI) or 2 ml (16-18 DPI) of Lactated Ringers' Solution given SC. Additionally, during 13-18 DPI, ML29 and MOPV groups received DietGel 76A, sterile $5 \%$ dextrose soaked mouse chow on the cage floor, and were housed with their cages placed half on a $42^{\circ} \mathrm{C}$ heating pad for thermal support.

In vivo Hartley outbred guinea pig experiments

Female Hartley outbred guinea pigs were ordered from Charles River (Willmington, MA, USA) with a requested weight of 250-300 grams. Upon arrival, all animals were randomized to study groups and allowed to acclimate for one week.

For vaccination and heterologous challenge, 3 animals were assigned to each group ( $\mathrm{n}=$ 3) and transferred to ABSL-3 housing. Animals in the ML29 group received a single dose of $10^{3}$ 
PFU of ML29 SC while mock vaccinated animals received PBS vehicle. Twenty-eight days following vaccination, animals were challenged with $10^{3} \mathrm{PFU}$ of the viscerotropic LCMV strain WE (LCMV-WE).

Guinea pigs were vaccinated with $10^{3}$ PFU of ML29 or a qRT-PCR determined equivalent dose of ML29P50. At 3, 7, and $14 \mathrm{DPI}$, four animals from each group were euthanized and blood was collected into $\mathrm{K}_{2}$ EDTA tubes for peripheral blood mononuclear cell (PBMC) isolation. PBMCs from whole blood were isolated with SepMate50 tubes containing Lymphoprep (STEMCELL Technologies Inc., Cambridge, MA, USA) and washed twice in RPMI 1640 (ThermoFisher \#11875093) supplemented with $1 X$ antibiotic-antimycotic, 1X GlutaMax, 10\% FBS, and 55 MM 2Mercaptoethanol (ThermoFisher \#21985023). Washed cells were used for ELISpot analysis as previously described [287]. Guinea pig IFN- $\gamma$ capture and biotinylated detection antibodies were a generous gift from Professor Hubert Schäfer (Robert Koch Institute, Berlin, Germany). Peptide cocktails containing a mixture of LASV strain Josiah glycoprotein precursor epitopes at a final concentration of $10 \mu \mathrm{M}$ were used for specific stimulation. Spots were read and quantified on a S5 UV ELISpot reader using BioSpot and ImmunoSpot software (Cellular Technology Limited, Cleveland, OH, US).

Blood collected in lithium-heparin microtiter tubes with a gel separator was spun at $6,000 \times g$ for 90 seconds. Plasma was separated and $100 \mu \mathrm{l}$ was loaded onto a Basic Metabolic Panel Plus reagent disk, and run on a Piccolo Xpress analyzer (Abaxis, Union City, CA, US). A group of 3 unvaccinated guinea pigs challenged with 10 PFU of LCMV-WE provided blood for comparison. Samples from mock vaccinated animals were pooled from 11 total blood draws (separated by at least a week) from 6 different guinea pigs and the exact same 11 data points are presented as a comparison basis to both day 7 and 14 samples. 


\section{Results}

VeroML29P53 cells interfere with replication of $r 3 L C M V-A r m-G F P / G F P$

We have previously documented that persistently infected VeroML29P15+ cells lost their ability to produce infectious ML29 “plaques" under agarose overlay [204]. Notably, this interference was also associated with particles generated into culture medium of persistently infected cells. The homologous interference is one of the major features of DIPs. Additionally, the ML29-persisitently infected cells do not produce plaques when superinfected with genetically closely-related arenaviruses, LASV, MOPV, and LCMV. LASV and MOPV are parental viruses used to generate infectious reassortant ML29, one of the most promising LASV vaccine candidates [18]. ML29 contains the MOPV L-segment encoding the $L$ and $Z$ proteins, and the LASV/Jos S-segment encoding GPC and NP [18]. In mini-genome systems, the NPs and L proteins of LASV and MOPV were exchanged without loss of function [288]. Meanwhile, the LCMV L protein required homologous NP for activity [288], and co-infection of cells with LASV and LCMV failed to generate infectious reassortants (Lukashevich, unpublished). To investigate heterologous interference of VeroML29PX cells further, these cells were infected with trisegmented LCMV-Arm (r3LCMV-Arm-GFP/GFP). In this system, the level of replication and/or interference can be observed based on the inhibition of expression of the GFP reporter gene. As seen in Figure 9, in wild-type Vero cells, replication of r3LCMV-Arm-GFP/GFP resulted in strong expression of reporter gene in time-dependent manner. In contrast, in VeroML29P28 and VeroML29P53 cells, expression of the reporter gene was heavily suppressed, and only a few cells were GFP-positive in the VeroML29P53 infection. These results confirm our previous observation that VeroML29PX cells interfere with replication of LCMV [1], and this interference can be measured either with classical infectious plaque assay, or by the expression of a reporter gene cloned/expressed in NP or GPC open-reading frames as in r3LCMV-Arm-GFP/GFP. 
Sub-genomic RNA species in VeroML29PX cells

Generation of deleted genomes during accumulation of DIPs (or interference activities in persistently-infected cells) is another major biological feature of DIPs [263]. Historically, sedimentation patterns in sucrose gradients of RNA isolated from interfering particles produced by LASV-persistently infected Vero revealed heavy deletions in the 28-31S sedimentations classes containing $28 \mathrm{~S}$ host ribosomal RNAs and LASV L RNA species [234]. High resistance of interference activity of LASV DI genome to UV-inactivation also suggested genomic deletions [49]. Here, we applied NB analysis to detect RNA deletions associated with L-segment of DIGs from ML29 infected cells using an L RNA-derived radioactive probe. NB analysis allows native viral RNA detection without any potential PCR biased enhancement of "helper" genomes in the initial RNA samples. For NB analysis, three types of RNA samples were used: (i) RNA from cells infected with ML29 at low MOI (acute infection control); (ii) RNA from LASV- and MOPV-infected cells (low MOI); and (iii) RNA from ML29-persisitently-infected cells, VeroML29P28 and -P53. As expected, in cells infected with LASV and MOPV at low MOI, the predominant RNA band was the L RNA migrated in the gel with predicted molecular mass, $7.2 \mathrm{~kb}$. In ML29-infected cells (at low $\mathrm{MOI}$ ), in addition to the high-molecular RNA species migrating at the position of $L$ RNA, several smaller RNA species were detected with the L RNA-derived probe. The predominant species gradually accumulated during 2-4 days after infection and migrated as heterogeneous bands in the area of $1.5 \mathrm{~kb}$ (Figure 10a). The low/undetectable (by plaque assay) level of ML29 replication in persistently-infected cells reasonably generated a weak signal in our NB analysis. Nevertheless, a full-length L RNA can be detected in VeroML29P28 and -P53 cells. In VeroML29P53 cells, in addition to L RNA, two additional RNA bands were detected with the L RNA probe. The predominant band migrated as an RNA species with a length of $\sim 3 \mathrm{~kb}$. In 
addition, a tiny band migrated in the gel slightly slower than $1.5 \mathrm{~kb}$ RNA species can be also faintly detected.

MGs derived from the LCMV L RNA failed to interfere with replication of infectious homologous virus

Two artificial constructs of the LCMV L-Segment with fluorescent reporter replacement of the complete L-gene (LCMV\#1074, Figure 11a top) or all but $198 \mathrm{Bp}$ of the L-gene (LCMV\#1076, Figure 11a bottom) were designed to test whether deletions in the L gene would result in successful competition (interference) with the replication of wild-type LCMV. Both of the constructs were replicated by the LCMV rescue system, and produced their respective fluorescent reporters (Figure 11b, left two panels, LCMV\#1074 top, LCMV\#1076 bottom). Additionally, LCMV-Arm infection was sufficient to provide the necessary trans-acting factors for replication, as seen by the fluorescent reporter produced from infection with $0.1 \mathrm{MOI}$ (Fig $11 \mathrm{~b}$, center panels) or $0.5 \mathrm{MOI}$ (Fig 11b, right panels) of LCMV-Arm. However, similar viral titers were isolated from the supernatants of cells transfected with LCMV\#1074, LCMV\#1076, or an irrelevant vector driving GFP production, at one day post LCMV infection (Figure 11c). This suggests that these constructs are insufficient to cause interference with in vitro viral replication.

\section{ML29P50 is attenuated in two mouse models}

One of the possible mechanisms of DIP-associated interference can be induction of innate (e.g., by RIG-I-dependent pathways triggered by pathogenic RNA species) and acquired immunity. Indeed, potent immunogenic features of DIPs are well documented for several RNA viruses and seems to contribute to vaccine efficacy [272]. Taking into consideration detection of substantial amount of L-derived RNA species in ML29 generated at low and high MOI (in 
persistently-infected ML29 cells) we used two small animal models CBA/J mice and STAT-1-/mice to test attenuation of ML29 and possible contribution of interfering particles. CBA/J mice are susceptible to both LASV and ML29 IC challenge $[17,165]$. The CBA/J IC challenge model was also used to test the attenuation of ML29 enriched with DIPs, ML29P50.

All mice challenged with ML29 succumbed to infection at 7DPI, while all mice receiving ML29P50 survived two weeks following challenge (Figure 12a). Additionally, ML29P50 challenge mice did not show weight loss or the clinical manifestations caused by ML29 challenge at 6-7 DPI. Viral loads in brain tissue measured by qRT-PCR at 7 DPI was undetectable for ML29P50 challenged mice (data not shown).

Mice deficient for STAT-1 do not respond to either type I or type II interferon and are therefore extremely susceptible to viral infection [289]. Likewise, STAT-1 ${ }^{\%-}$ mice are susceptible to LASV infection [198]. These mice can be also used to distinguish between lethal and nonlethal human isolates of LASV. In addition STAT-1-/- mice surviving LASV infection show sensorineural hearing loss similar to that observed in human LASV survivors [216]. STAT-1 ${ }^{-1}$ mice are also susceptible to infection caused by ML29 and MOPV [204]. Because supportive measures are commonly used for human Lassa fever (LF) patients [290], we attempted to use supportive measures to increase the survival of STAT $-1^{-/-}$mice following ML29 vaccination to examine the potential of the vaccine to cause sensorineural hearing loss. Supportive measures including fluid administration, caloric support, and temperature support during hypothermia for mice infected with ML29 or MOPV. While the efforts were largely unsuccessful with $1 / 12$ ML29 and 3/12 MOPV mice surviving (Figure 12d), survivors did not show weight loss (Figure 12e) or clinical manifestations (Figure 12f) that lasted more than 3 weeks following infection. This allowed for hearing tests by audible startle to check for signs of sensorineural hearing loss. No 
hearing loss was observed in ML29, ML29P50, or MOPV mice at 62 DPI (Figure 12g-i) under the same conditions used to detect LASV associated hearing loss [216].

\section{ML29 induces LASV-specific T cell responses in Hartley outbred guinea pigs}

Mice provide useful tools to study arenaviruses. However, mice cannot mimic the pathogenesis of the human diseases caused by these viruses. Guinea pigs with different genetic backgrounds are a more appropriate model for the study of LASV-induced pathogenesis and mechanisms of the protection. Hartley outbred guinea pigs are susceptible to infection with LCMV-WE with a LD50 less than 10 PFU, and display similar disease to those infected with LASV [209, 291, 292]. Hartley guinea pigs were used to study the anticipated cross-protection induced by ML29. The rationale of this study was based on the protection of Rhesus macaques vaccinated with ML29 against fatal LF-like disease caused by LCMV-WE [161]. As seen in Figure 13a, at day 10, LCMV-WE also killed all Hartley guinea pigs infected with LCMV-WE. While vaccination with ML29 did not protect against fatal disease caused by LCMV-WE, we still observed a substantial delay in time of death for vaccinated animals. Furthermore, ML29 vaccinated guinea pigs displayed LASV GPC specific T cell responses at 14 days following vaccination (Figure 13b). Their responses were measured in IFN- $\gamma$ ELISPOT after stimulation of PBMC cells with peptide cocktails derived from LASV/Jos GPC, as previously described [204].

Guinea pigs are also commonly used animals for pre-clinical toxicological studies to assess safety of biologics (vaccines and therapeutics). Neither ML29 nor ML29P50 treated animals had hematological signs of renal disease (Figure 13c-f) or viral hepatitis (Figure 13g-j) commonly seen in LF patients. However, LCMV-WE induced signs of renal disease observed in guinea pigs meeting endpoint criteria (EC) including elevated glucose, blood urea nitrogen, amylase, and decreased calcium (Figure 13c-f). LCMV-WE also induced viral hepatitis observed 
by increased Alanine Aminotransferase (ALT) and Aspartate Aminotransferase (AST) and decreased albumin in EC guinea pigs. Interestingly, albumin was also significantly decreased at 7DPI and there was a non-significant trend towards increased Gamma-Glutamyl Transferase at this time.

\section{Discussion}

Previously we documented that Vero cell persistently infected with ML29 after passage 15 (VeroML29P15) did not generate infectious plaques under agarose overlay, a standard assay to quantitate replication-competent (infectious) viruses. In addition, these cells did not form plaques after super-infection with closely-related viruses, MOPV and LASV, and LCMV. While interference between ML29, MOPV, and LASV was highly expected, experiments in MG systems indicated that LCMV L protein requires specific (homologous) NP for MG function [288]. This finding is in line with the observed failure to generate live reassortants in cells co-infected with LCMV and LASV (Lukashevich, unpublished). To investigate further the heterologous interference of VeroML29P cells, we used tri-segmented LCMV expressing the GFP reporter gene, r3LCMV-Arm-GFP/GFP. In agreement with our infectious plaque assay results, ML29P28 and ML29P53 cells interfered with replication of r3LCMV-Arm-GFP/GFP since the expression of the GFP reporter following infection with r3LCMV-Arm-GFP/GFP was significantly reduced. Greater interfering activity was seen in the VeroML29P53 cells, which tended to have approximately a 10- to 100-fold reduction in the number of GFP-positive cells infected at both 24 and 48 hours. In VeroML29P28 cells, the total number of infected cells was more similar to that observed in standard Vero cells, however there was diminished average expression of the GFP in each infected cell. This is consistent with the observation that there is less viral protein (particularly GPC and NP) detected per cell by immunofocus assay in ML29P34 cells compared to cells with primary ML29 infection [204]. 
Interestingly enough, in NB analysis with an L RNA-derived probe we observed numerous small RNA species with a size ranging from about $1 \mathrm{kBp}$ to $4 \mathrm{kBp}$ along with the expected viral genome and L mRNA. These L RNA-derived small RNA species were predominant in ML29-acutely infected cells. Notably, the S RNA-derived probe detected RNA species with expected size (viral S RNA and mRNA for NP). While the detection of small L RNA-derived species in ML29-infected cells infected at low MOI was a surprising finding, these small RNA species can contribute to enhanced immunogenicity of ML29. Small viral RNAs can be potent activators of RIG-I, MDA-5 or PKR [253]. We are assuming that part of the mechanism of advanced attenuation and/or immunogenicity of ML29 compared to MOPV is these small RNA species causing the activation of antiviral innate immunity at very early stages of the infection. This is very crucial to viral control and building potent acquired immune responses at latter stages of the infection. It has been documented that the L and NP genes from LASV and MOPV are interchangeable in a MG system [288]. However, LCMV L is not functional with LASV nor MOPV NP, although both LASV and MOPV L support MG replication with LCMV NP [288]. Importantly, most previously described arenavirus MG systems contained the cis-acting elements from the viral S RNAsegments $[181,288]$. In these MG systems, NPs and L proteins of LASV and MOPV could be exchanged without loss of function. Still, the MOPV MG was about 3-fold less active than the LASV MG, irrespective of NP and L origin. This means that cis-acting elements (e.g., MOPV 3'UTR) contribute to MG activity. Design and functional testing of MG systems based on the L RNA-derived cis-acting elements will provide very valuable insights regarding the replication machinery of these arenaviruses. Based on available experimental results and our data, we hypothesize that mismatch between the either LASV NP and MOPV L, or LASV NP and the cis-acting elements from the MOPV/ML29 L-segment are responsible for the generation of small RNA species in cells infected with ML29 at low MOI. Notably, this mismatch does not 
affect replication efficiency of ML29 since replication kinetics in vitro was very similar for ML29 and parental viruses, LASV and MOPV. It is also possible that single nucleotide polymorphisms in ML29, particularly mutations in L protein, Y851N, R1233G, and D2136N [17], account for different binding ML29 NP affinity with either ML29 L or the cis-acting elements of ML29.

Our search for "classical" defective genomes with RNA deletions in ML29-persistentlyinfected cells resulted in the detection of a unique RNA band with a size slightly greater than 3 $\mathrm{kb}$ in addition to the viral L RNA species. This band was observed only in persistently infected cells using a NB probe derived from the L RNA. This band was predominant, at least in VeroML29P53 cells, and can be also detected in VeroML29P28 cells (after longer exposure of NB to the film, Fig 10a). In general, defective viral genomes are naturally generated MG systems preserving terminal sequences required for replication/packaging with substantial deletions of internal parts of the genome. These natural MG can be used as potential broad-spectrum antivirals for rapidly evolved (highly heterogeneous) RNA viruses (e.g., influenza) [293]. Indeed, defective interfering RNAs inhibited influenza virus replication in human respiratory tract cells [294]. This approach can be also attractive for arenaviruses. LCMV and LASV are the most genetically diverse mammalian arenaviruses. Genetic diversity is one of the problems for development of universal LASV vaccine. To test potential antiviral activity of arenavirus MGs, we used recombinant LCMV L RNA-derived plasmids with deleted L protein. These plasmids, LCMV\#1074 and LCMV\#1076, contained native 3' and 5'UTR sequences, IG region and expressed GFP instead of large, L protein. However, transfection of Vero cells with these plasmids did not affect replication of LCMV (Figure 11b). Further experiments are needed to assess role of defective MGs in arenavirus replication. Nevertheless, experiments with LCMV\#1074 and LCMV\#1076 MGs provide evidence that the cis-acting elements from the L-segment can be successfully used to design new arenavirus MG systems. 
While we currently do not how or if the L RNA-derived small RNA species, (either from acute infection of persistently-infected cells), contribute to advanced attenuation and immunogenicity of ML29, we provided evidence that indeed ML29P50 was deeply attenuated in $\mathrm{CBA} / \mathrm{J}$ and STAT $-1 \%$ mice. In the future, it may be interesting to see if a blended vaccine formulated with interfering particles produced by persistently-infected cells will induce broad cross-protection against LASV strains from different phylogenetic lineages of LASV. In the STAT$1^{-/}$mouse model, ML29 was more attenuated than MOPV. ML29 enriched with interfering particles (ML29P50) induced potent T cell responses, and was fully attenuated in these mice.

ML29P50 inoculated STAT-1 ${ }^{--}$mice had weight loss and clinical manifestations in the week following vaccination (Figure 12e,f) but quickly recovered. By the end of the two-month observation, their weights were similar to mock treated mice. Supportive care was largely unsuccessful in increasing the survival of ML29 inoculated STAT-1 ${ }^{--}$mice, but did rescue $25 \%$ of animals challenged with a lethal dose of MOPV. There were no signs of sensorineural hearing loss with ML29, MOPV, or ML29P50 mice two months following inoculation, confirming that this is not likely to be an adverse effect of ML29 vaccination.

Arenaviruses, by definition, are rodent-borne viruses. These viruses are treated differently by the immune system of rodents and humans (or NHPs). Nevertheless, among experimental rodents, guinea pigs seems to be the most reliable and appropriate small animal model to study arenavirus-induced pathogenesis and protective immune responses. We used outbred Hartley guinea pigs in efforts to assess safety and cross-protective immunogenicity of ML29. Hartley guinea pigs vaccinated with ML29 and challenged with lethal LCMV-WE (which mimics LF in NHPs), were not protected. However, they still had had a modest (but statistically significant) delay in time to death following LCMV-WE challenge. In contrast, ML29 vaccinated rhesus macaques were fully protected from lethal LCMV-WE challenge [161]. This is likely due to 
the unusually high susceptibility of these Hartley guinea pigs to LCMV-WE infection despite the clear presence of T-cell immunity by ELISpot (Figure 13b.) It is also possible that different T-cell epitopes are protective in the Hartley guinea pig model, and that these epitopes do not provide for heterologous protection against LCMV-WE. Challenge with guinea pig adapted LASV/Jos may be a more appropriate challenge model to evaluate ML29 protection. While LCMV-WE infection induced clear signs of renal disease (elevated blood glucose, blood urea nitrogen and amylase with reduce calcium) and viral hepatitis (elevated ALT, AST, GGT and reduced albumin), ML29 and ML29P50 did not. This further demonstrates the excellent safety profile of these live attenuated vaccines. 


\section{Figures}

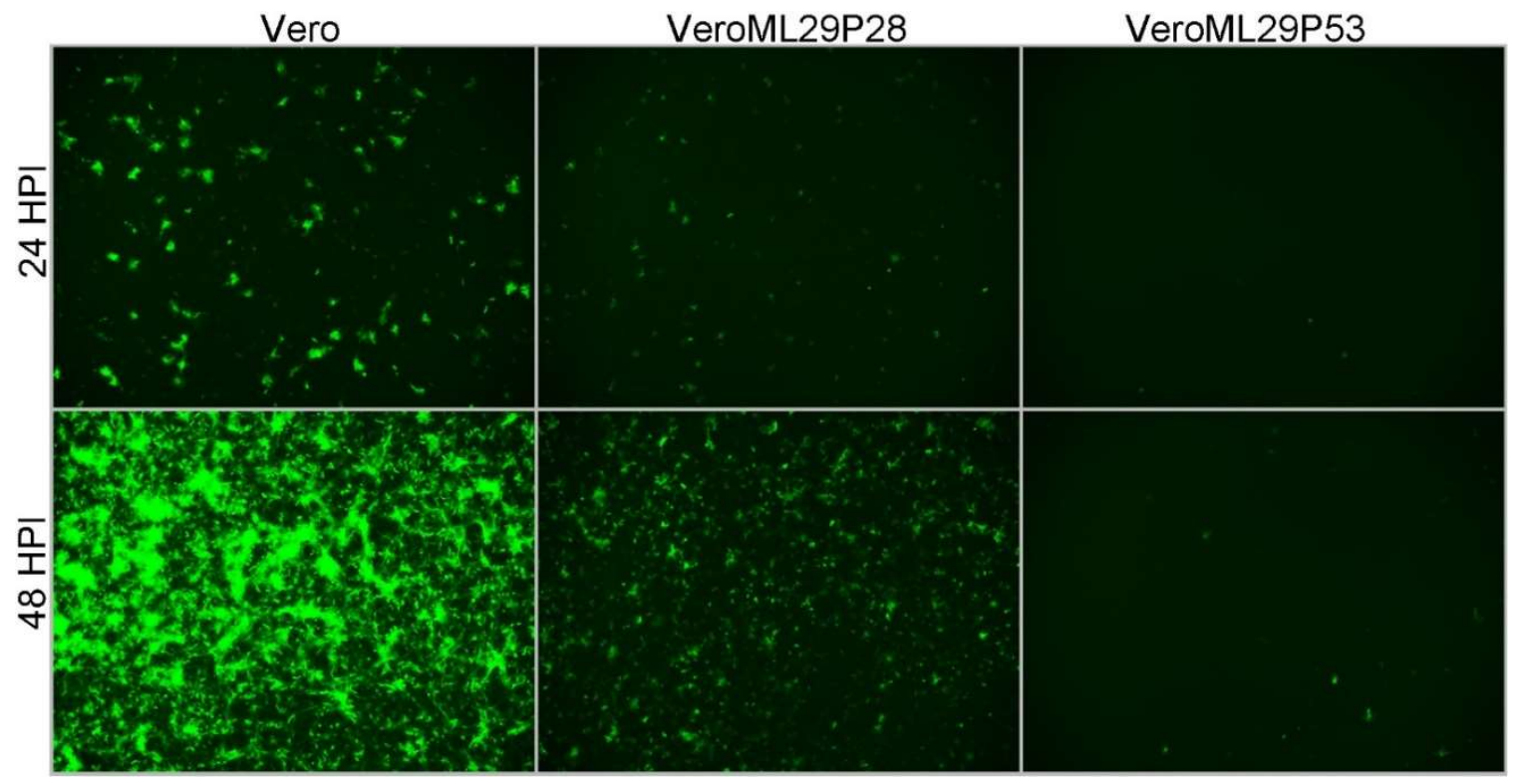

Figure 9. GFP expression from Vero (left), VeroML29P28 (center) and VeroML29P53 (right) cells infected with $3.4 \times 10^{4} \mathrm{FFU}$ of r3LCMV-Arm-GFP/GFP at 24 (top) and 48 (bottom) hours post infection.

(a)

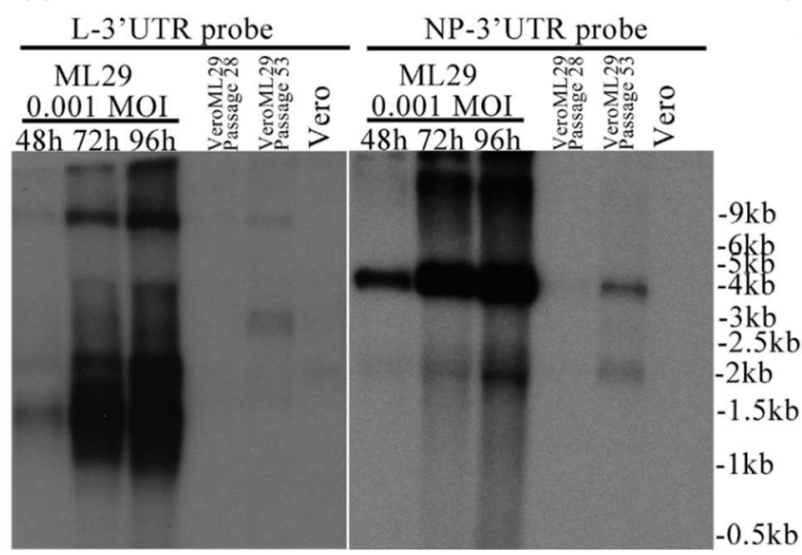

(b)

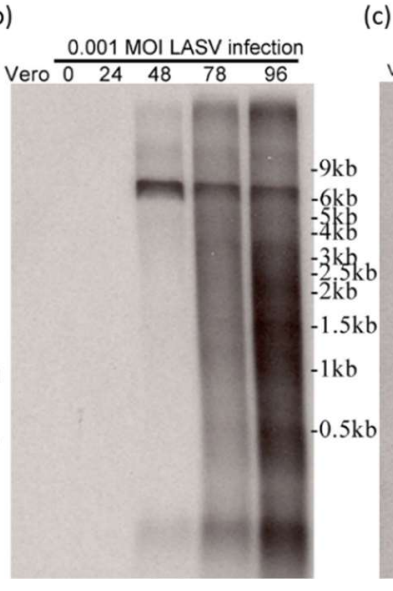

c) Vero \begin{tabular}{llll} 
& \multicolumn{4}{c}{0.001 MOI MOPV infection } \\
\cline { 2 - 4 } & $48 \quad 78 \quad 96 \quad 120$
\end{tabular}

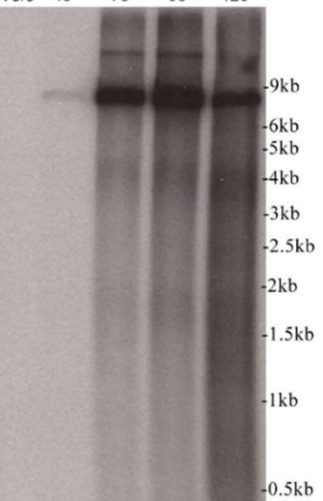

Figure 10. (a) Northern blot of Vero cells infected with a $0.001 \mathrm{MOI}$ of ML29 at the indicated hours post infection (Lanes 1-3), VeroML29P28 at 96 hours after passaging (lane 4), VeroML29P53 at 96 hours after passaging (lane 5), and uninfected Vero cells (lane 6) detected 
with a probe targeting the N-terminus of the ML29/MOPV L-protein coding region extending into the non-repeated portion of the L-Segment 3' untranslated region (left blot) or a probe targeting the N-terminus of the ML29 NP-protein coding region extending into the non-repeated portion of the S-Segment 3' untranslated region. (b) Norther blot of Vero cells (uninfected, lane 1) infected with a $0.001 \mathrm{MOI}$ of LASV at the hours post infection indicated (lane 2-5) detected with a probe targeting the $\mathrm{Z}$ protein coding region of the L-segment. The 0 HPI lane was collected immediately following infection. (c) Northern blot of Vero cells infected with a 0.001 MOI of MOPV at the indicated hours post infection (Lanes 2-4) and uninfected Vero cells (lane 1) detected with the same probe used in (a).

(a)
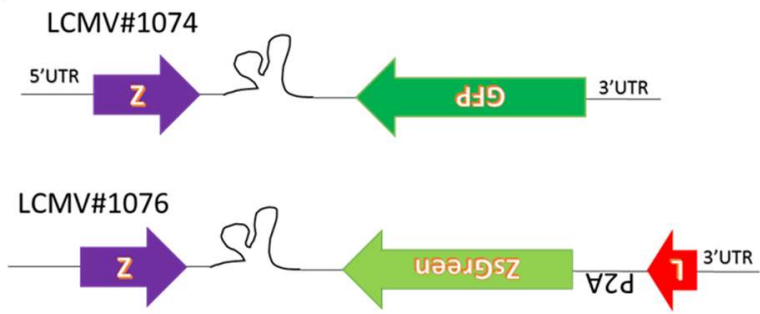

(b)
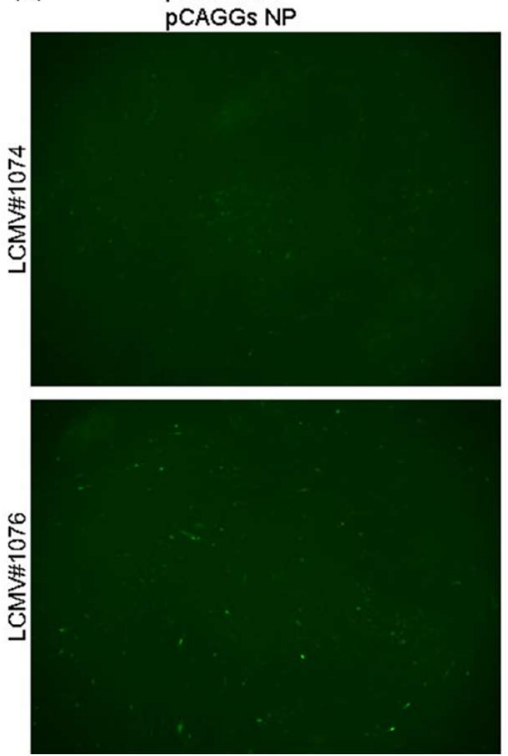

$0.1 \mathrm{MOI}$ (c)
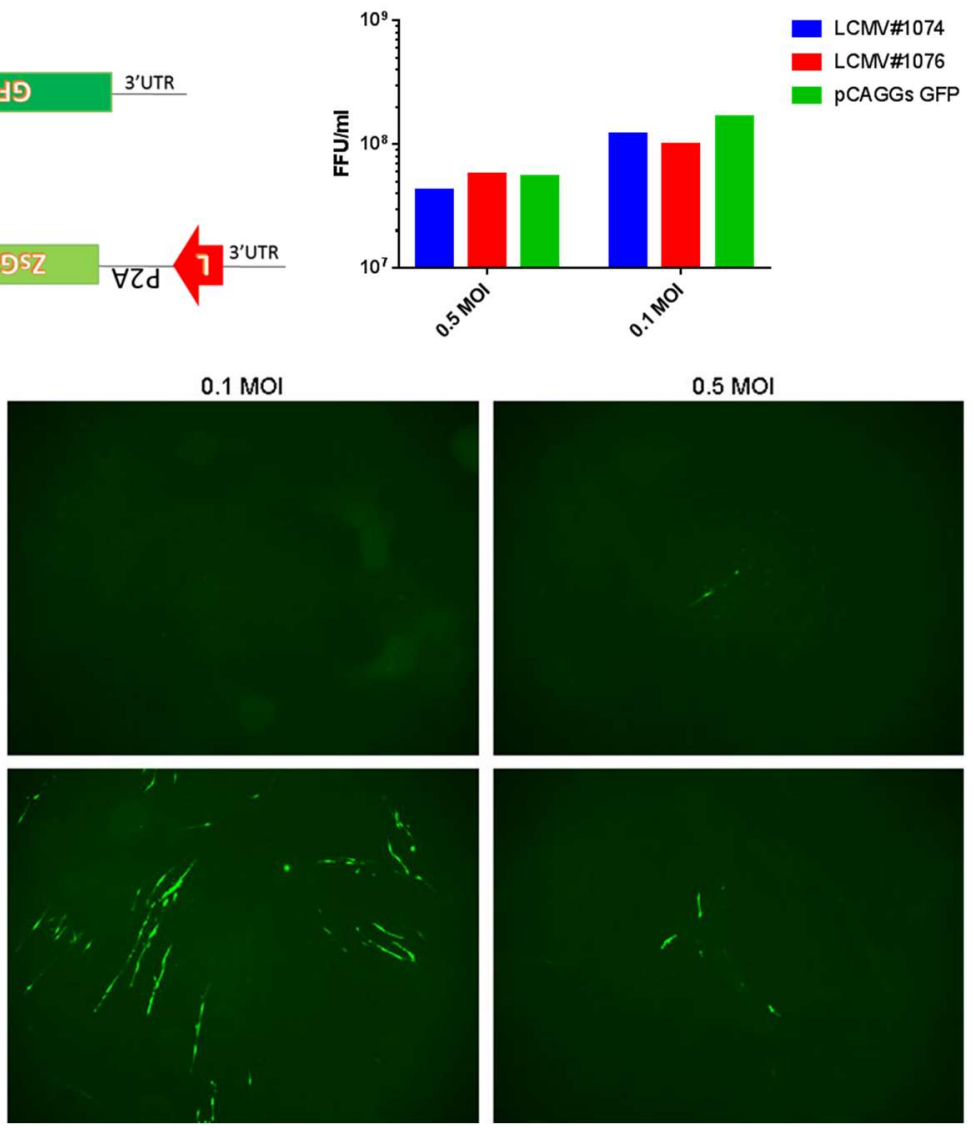
Figure 11. (a) Schematic showing the constructs LCMV\#1074 and LCMV\#1076 which were inserted into an mPol-I driven expression vector. (b) BHK-21 cells were transfected with mPollLCMV\#1074, mPoll-LCMV\#1076, or a pCAGGs expression vector for GFP. One day later, the cells were infected with LCMV-Arm at an $\mathrm{MOI}$ of either 0.1 or 0.5 and allowed to incubate for an additional day before the supernatants were collected and titered by focus forming assay. (c) Left two panels: BHK-21 cells were transfected with LCMV\#1074 (top) or mPoll-LCMV\#1076 (bottom) and expression vectors for the two LCMV trans-acting replication factors: RdRP Lprotein (pCAGGs L) and nucleoprotein (pCAGGs NP). Right four panels: Vero cells transfected with LCMV\#1074 (top) or mPoll-LCMV\#1076 (bottom) and, one day post transfect, infected with a $0.1 \mathrm{MOI}$ (center two panels) or $0.5 \mathrm{MOI}$ (right two panels) of LCMV-Arm. Images captured 2 days post transfection.

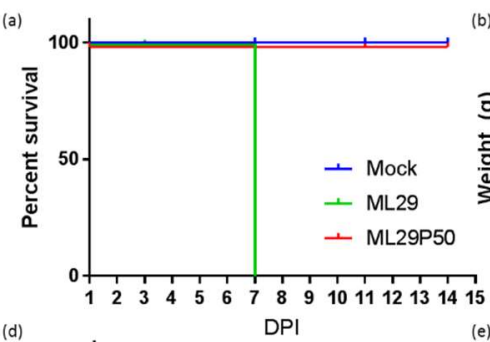

(d)
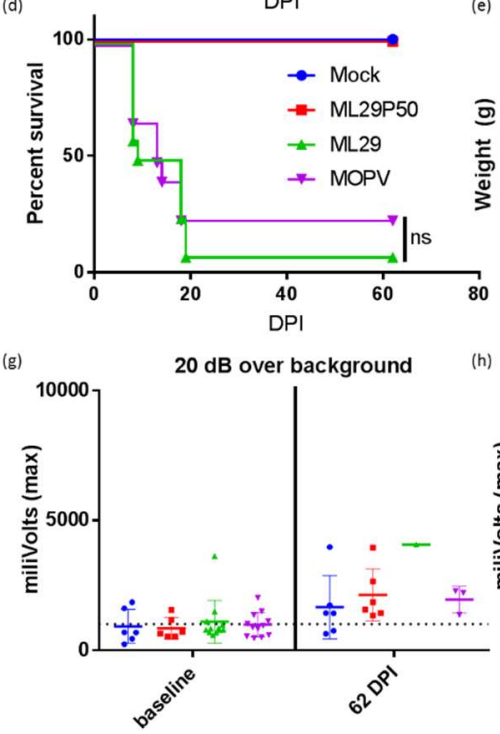
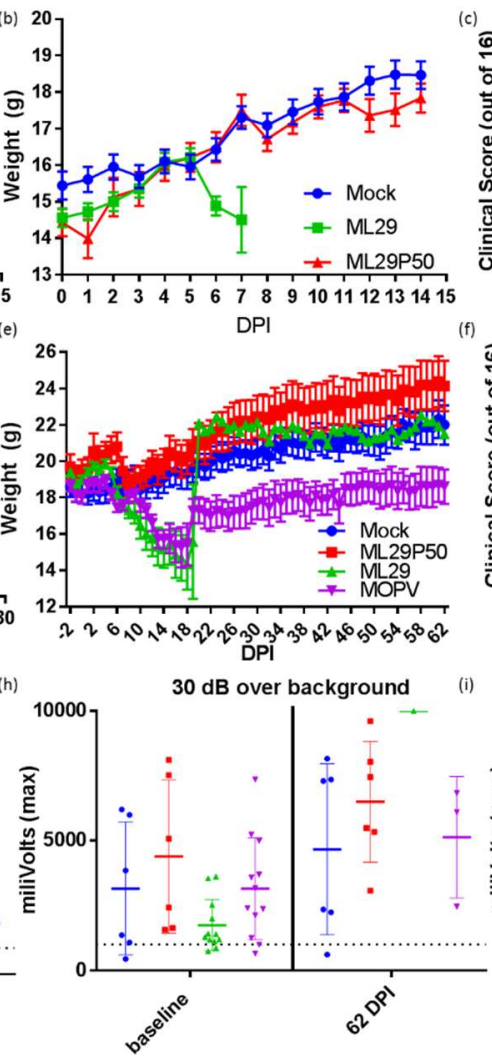
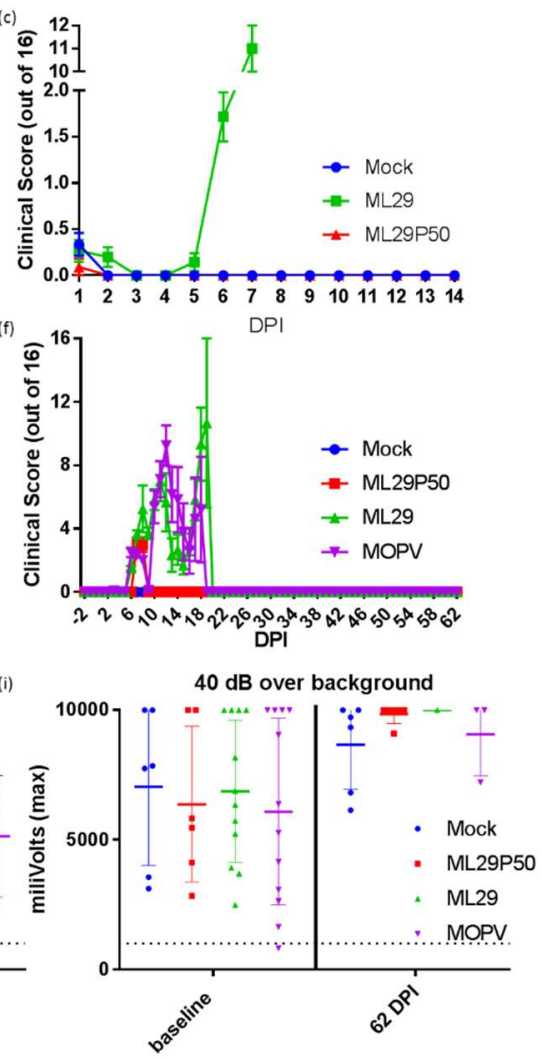
Figure 12. CBA/J mice were inoculated IC with $10^{3}$ PFU of ML29 (green), a qRT-PCR equivalent dose of ML29P50 (red), or mock inoculated with PBS (blue) and followed for two weeks for (a) survival, (b) weight, and (c) clinical manifestations. Survival curves are significantly different $(\mathrm{p}<$ 0.0001 ) by Mantel-Cox Log-rank test. STAT-1 ${ }^{-1}$ mice were inoculated IP with $10^{3}$ PFU of ML29 (green), a qRT-PCR equivalent dose of ML29P50 (red), 10³ PFU of MOPV (purple), or mock inoculated with PBS (blue) and followed for two months for (d) survival, (e) weight, and (f) clinical manifestations. Sixty-two days post inoculation, mice were tested for hearing loss by acoustic startle with a sound generated at (g) $20 \mathrm{~dB}$, (h) $30 \mathrm{~dB}$, or (i) $40 \mathrm{~dB}$ greater than the background noise of the room. Following the startle tone, the movement of the mouse was tracked with a digital pressure transducer, which recorded pressure signal as millivolts. The dashed line represents the sensitivity threshold used to distinguish response to the sound from non-specific signal. Values above the dashed line were able to hear the startle inducing tone.
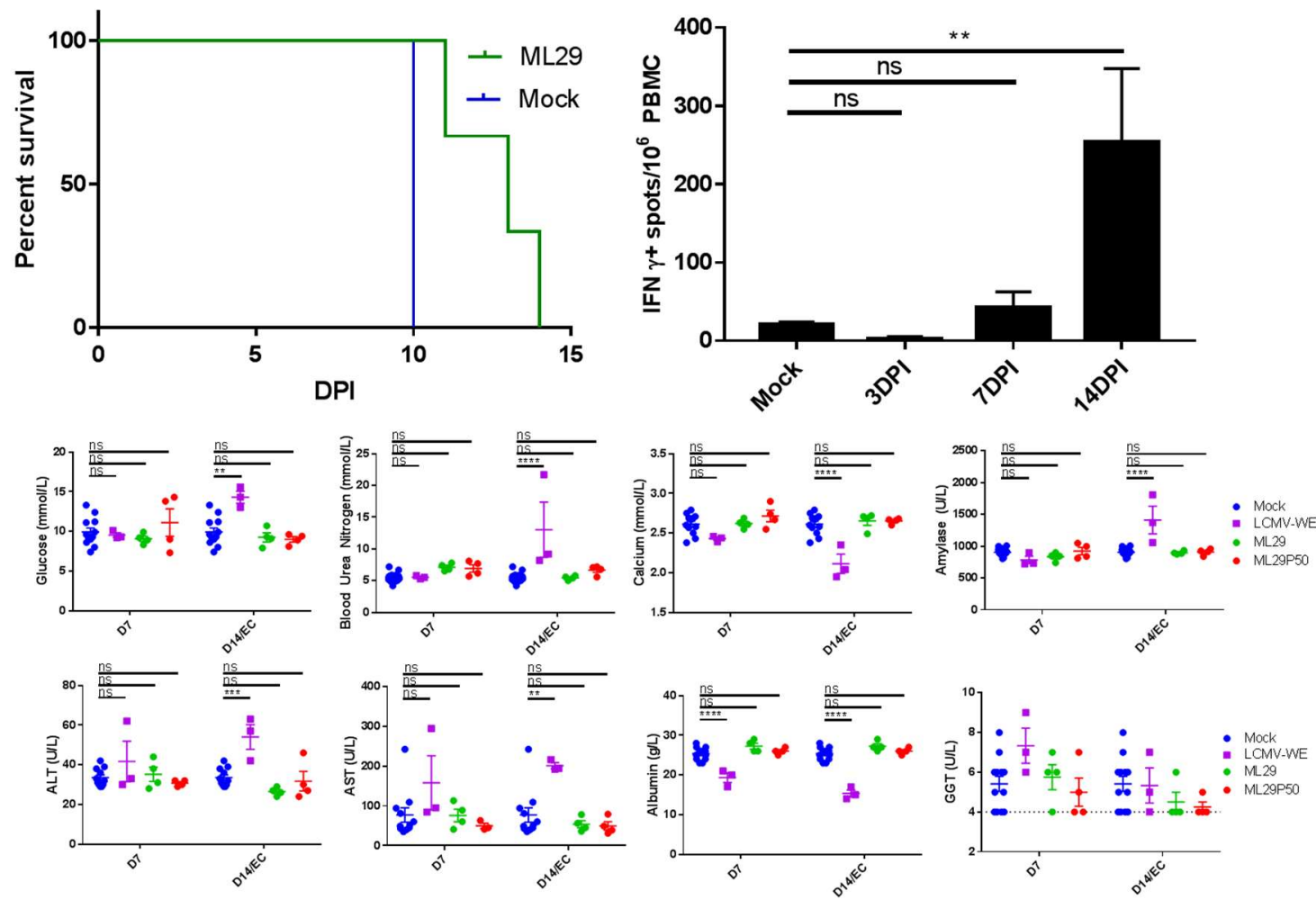
Figure 13. (a) Hartley guinea pigs were vaccinated with $10^{3}$ PFU of ML29 SC or mock vaccinated. Twenty-eight days post vaccination, mice were challenged with $10^{3}$ PFU of LCMV-WE SC and followed for survival. Survival curves are significantly different $(p=0.0253)$ by Mantel-Cox Logrank test. (b) Hartley guinea pigs ( $n=4$ per time point) were inoculated with ML29. Blood was collected and PBMCs were tested for IFN- $\gamma$ production in response to LASV/Jos peptide stimulation by ELISpot at 3,7 , and $14 \mathrm{DPI}{ }^{* *} \mathrm{p}<0.01$ by one way ANOVA with Dunnett's posthoc comparison to mock. (c-j) Blood chemistry values measured with a Piccolo Xpress analyzer including (c) glucose, (d) blood urea nitrogen, (e) calcium, (f) amylase, (g) ALT, (h) AST, (i) Albumin, and (j) GGT. ${ }^{* *} \mathrm{p}<0.01, * * * \mathrm{p}<0.001, * * * * \mathrm{p}<0.0001$ by one way ANOVA with Dunnett's post-hoc comparison to mock. 


\section{CHAPTER V}

\section{ADVANCED SAFETY AND GENETIC STABILITY IN MICE OF A NOVEL DNA-LAUNCHED VENEZUELAN EQUINE ENCEPHALITIS VIRUS VACCINE WITH REARRANGED STRUCTURAL GENES ${ }^{2}$}

\section{Introduction}

Venezuelan Equine Encephalitis Virus (VEEV) is member of the genus Alphavirus in the family Togaviridae. There are six antigenic subtypes and seven varieties in the VEEV complex, most of which are endemic to Central and South America $[295,296]$. Types IAB and IC are associated with epizootic outbreaks in equines, when the virus breaks from its sylvatic cycle between the mosquito vector and rodents or birds [295]. In equines, VEEV presents as a biphasic illness with high fever followed by neurologic disease, resulting in $50 \%-89 \%$ mortality [297]. During infection, equines have a high titer viremia, which can lead to subsequent transmission through the mosquito vector to other equines or humans [297]. However, person-to-person transmission has not been reported. In humans, VEEV causes a biphasic febrile illness followed by myeloencephalitis with high morbidity, including neurological complications, and a mortality rate of approximately $2 \%$ [298]. Significant overlap occurs between regions of VEEV endemicity and that of some other tropical disease, which complicates clinical diagnosis. In particular, it has been reported that overlap with areas of dengue virus infection may result in an underestimation of VEEV prevalence [295]. In a population-based study, the seroprevalence of

\footnotetext{
${ }^{2}$ This chapter is adapted from work published in Vaccines, 2020, Volume 8, Issue 1 with the same title under a Creative Commons Attribution (CC BY) license [27].
} 
VEEV has been reported to be as high as $75 \%$ in some endemic regions [299]. Climate change may be contributing to the increased geographical distribution of Culex mosquitos, increasing the population of humans and equine at risk for contracting VEEV [300-302].

VEEV was weaponized in the US and former USSR before biological warfare programs were discontinued [298, 303-305]. Still, VEEV is recognized as a potential biological weapon threat. In the US, VEEV subtypes IAB and IC are regulated under the HHS/USDA Select Agent program due to the severe threat posed to human and animal health, especially in the absence of FDAlicensed human vaccines and therapeutics [306]. Due to the minimum infectious aerosol dose required for infection, VEEV also has a long history of being implicated in laboratory-acquired infections [307-309].

The first effort to create a VEEV vaccine used a formalin inactivated wild-type virus. However, these experimental vaccines had low immunogenicity and were prone to incomplete inactivation [310]. A live attenuated vaccine, TC-83, was developed by serially passaging VEEV TrD through guinea pig heart cells 83 times while monitoring virulence by intracranial (IC) injection in mice [13]. VEEV TC-83 contains 10 point mutations compared to the VEEV TrD strain, however the attenuation is attributed to two key differences: $G$ to $A$ mutation at position 3 of the $5^{\prime}$ untranslated region, which reduces translation efficiency by restoring Ifit1 restriction, and a threonine to arginine substitution at position 120 of the E2 glycoprotein [311-313].

Murine infection with the Trinidad-Donkey strain (TrD) of VEEV has been identified as a good challenge model for vaccine development, in line with the FDA animal rule [314], in addition to the aerosolized model of infection in Rhesus macaques. TC-83 has been tested in human phase I and II trials and became available for individuals "at risk" through the Special Immunization Program under the supervision of the United States Army Medical Research Institute of Infectious Disease (USAMRIID, Fort Detrick, MD) [29]. TC-83 human trials have 
revealed high reactogenicity, often leading to symptomatic VEEV infection including febrile illness and headaches in up to $40 \%$ of vaccinated individuals [37, 315]. Additionally, it has been documented that a single dose of TC-83 is often insufficient to illicit protective humoral responses (neutralizing antibodies), as detected by plaque-reduction neutralization tests (PRNT). Individuals with poor responses are usually further immunized (boosted) with formalininactivated TC-83 (called C-84) to enhance PRNT titers following TC-83 vaccination [29]. Experimental V3526, a rationally designed VEEV vaccine containing a mutation in the E2 cleavage site of the structural polyprotein, showed promising results in mice. However, the phase I clinical trial revealed unacceptable adverse effects and development of V3526 was discontinued [316]. Recently, chimeric alphaviruses, either with Sindbis virus, or the insect hostrestricted Eilat virus, have been tested as VEEV vaccine platforms [37, 41, 317, 318]. An alternate approach has been proposed to create a novel vaccine by the introduction of an internal ribosome entry site to drive control of VEEV structural proteins or capsid [319-321].

The limited number of attenuating mutations in TC-83 and the high error rate of the viral RNA-dependent RNA-polymerase (RdRP) seem to be related to the instability of TC-83 attenuation [317]. Substantial progress in alphavirus molecular virology and VEEV pathogenesis has provided a variety of powerful tools to address the safety and immunogenicity of the VEEV TC-83 vaccine. Recently, we have used several approaches to further improve the VEEV TC-83 vaccine: (i) infectious DNA (iDNA) technology to launch a live-attenuated vaccine in vivo [322]; (ii) rearrangement of alphavirus structural genes; and (iii) genetic stabilization of the TC-83attenuated phenotype [44]. The novel V4020 experimental vaccine was rationally designed by securing the key TC-83 attenuating mutation (E2-T120A), along with a further improvement of attenuation by rearrangement of TC-83 structural genes [44]. 
In proof-of-concept studies, V4020 provided complete protection against wild-type VEEV TrD challenge, inducing sterilizing immunity in mice [44]. After a single dose of either subcutaneous live V4020 or electroporation with V4020 iDNA, one month later, BALB/c mice were protected from lethal challenge with VEEV-TrD [44]. Cynomolgus macaques, vaccinated with either a single dose or two doses of V4020, were aviremic following an aerosol challenge with VEEV TrD [323]. Here, we compared the safety and genetic stability of V4020 and TC-83 in viral replication kinetics studies in brain tissues, and then assessed the potential to revert to a pathogenic phenotype during passages of the vaccines in mice. In addition to virology methods, NanoString gene expression profiling and RNA-Seq analysis were applied to track viral genetics and host responses during the replication of both attenuated viruses in brain tissues of experimentally infected mice.

\section{Materials and Methods}

\subsection{Viruses}

VEEV TC-83 was obtained from U.S. Army Medical Research and Materiel Command (Fort Detrick, MD, USA), and amplified once in Vero cells. The pMG4020 plasmid for launching was previously described [44]. VEEV V4020 virus was prepared by electroporation of pMG4020 into Vero cells and the generated virus particles were concentrated by ultracentrifugation [44]. Virus titers were determined by infecting monolayers of Vero cells in 24-well tissue culture plates with serial dilutions of virus stocks for 1 hour, followed by methylcellulose overlay. Three days post infection, cells were fixed with paraformaldehyde and stained with crystal violet to count infectious plaques.

\subsection{Vaccine Replication Kinetics in Brain Tissues}


BALB/c mice (6-8 weeks old, female, 24 mice per group) were inoculated IC with $10^{5}$ plaque forming units (PFU) of either V4020 or TC-83 in a total volume of $20 \mu \mathrm{L}$ of PBS. To prepare for IC inoculation, mice were shaved, anesthetized with isoflurane in an induction chamber, transferred to a nose cone, had the injection site cleaned with first povidone iodine followed by ethanol, and positioned with their head tilted forward. A 27-guage needle (BD) was inserted through the foramen magnum angled to prevent damage to the brain stem. Following injection, mice were monitored during recovery. Mice that did not recover from the IC procedure or that met euthanasia criteria at 1 DPI (about 7\% of all IC treated animals with no apparent association to treatment group) were excluded from analysis.

Mice were weighted daily and assessed for signs of clinical disease on a 16-point scale as previously described [44]. Briefly, observation was based on grooming, adjusted grimace scale score, activity, and neurological responses including tail strength, hind leg strength, abdominal curl reflexes, and righting reflexes. On days 1, 2, 3, 5, 7, 9, 11 and 13-post infection, three mice from each group were sacrificed, and brain tissue was collected. Half of the brain was fixed in neutral buffered formalin for 24 hours before standard processing and paraffin embedding for histology. A quarter of the brain was placed in $2 \mathrm{ml}$ screw top tubes with $1 \mathrm{~mL}$ TRIzol Reagent (ThermoFisher \#15596026), and homogenized with glass beads in a bead beater for isolation of RNA by phenol-chloroform extraction. The remaining brain tissue was homogenized in cell culture media and clarified as described previously [204] for plaque assay.

\subsection{Sequential IC Passages in Mice}

BALB/c mice (6-8 weeks old, female, 10 mice per group) were inoculated IC, and, two days post infection, two mice from each group were sacrificed, brain tissue was collected, homogenized, clarified, aliquoted, and stored at $-80^{\circ} \mathrm{C}$, as described above. One aliquot from 
each mouse was titered by plaque assay. A second aliquot was used to prepare the inoculum for subsequent passages after a single freeze-thaw cycle. Each mouse was inoculated with $10^{5}$ PFU of virus comprised of $5 \times 10^{4} \mathrm{PFU}$ per dose originating from each of two mice from the previous passage. The remaining mice in each group were weighed daily and monitored for clinical signs for 14 days or until they met the euthanasia criteria of $25 \%$ weight loss or the loss of ability to obtain food and water for a period of 24 hours or greater (e.g., complete loss of righting reflex or hind leg paralysis.) At the time of sacrifice, brain tissue was collected as described above.

\subsection{In Situ Hybridization and Histology}

Formalin-fixed paraffin embedded (FFPE) brain tissue was sectioned on a Leica RM2125 RTS microtome to a thickness of approximately $5 \mu \mathrm{m}$ and mounted on charged glass slides. Slides were baked to dry. Manual hematoxylin and eosin staining was performed as previously described [324]. In situ hybridization was done using RNAScope HD Assay Brown (\#322300) using a probe targeting VEEV nsP3 (\#404501, Advanced Cell Diagnostics, Hayward, CA, USA) according to the manufacturer's directions.

\subsection{NanoString Array}

RNA collected during necropsy from the kinetic study on days $5,7,9,11$, and 13 post inoculation was isolated from mouse brain homogenized in TRIzol as described above. RNA was similarly collected from mock (PBS) IC-inoculated mice. Purified RNA was quantified on a NanoDrop 2000 spectrophotometer (ThermoFisher, Wilmington, DE, USA), diluted to $10 \mathrm{ng} / \mu \mathrm{L}$, and sent to NanoString Technologies (NanoString Technologies, Seattle, WA, USA) for analysis. A total of $100 \mathrm{ng}$ of each sample was hybridized to the nCounter Mouse Neuro-inflammation panel (XT-CSO-MNROI1-12) for 16 hours at $65^{\circ} \mathrm{C}$. Hybridized RNA was quantified on a NanoString Prep Station and Digital Analyzer Max System (NanoString Technologies, Seattle, 
WA, USA) with the manufacturer's high sensitivity protocol. Additional probes to detect VEEV genes (Table 2) were designed and synthesized by NanoString Technologies (NanoString Technologies, Seattle, WA, USA) based on the TC-83 GenBank reference sequence L01443.1. These probes were additionally included in the neuro-inflammation panel.

\subsection{RNA-Seq}

Brain tissue from mice inoculated with passages one through five (P1-P5) of V4020 and TC83 were collected two days post inoculation, DNAse I treated (Zymo Research \#E1010), and

Table 2. NanoString probes used for the detection of Venezuelan Equine Encephalitis Virus (VEEV) genes.

\begin{tabular}{ll}
\hline Target & Probe \\
\hline $6 \mathrm{~K}$ & $\begin{array}{l}\text { ACAAT AACCA ACAGA TGTTC TGGAT TCAAT TGCTG ATCCC TCTGG CCGCC TTGAT } \\
\text { CGTAG TGACT CGCCT GCTCA GGTGC GTGTG CTGTG TCGTG CCTTT }\end{array}$ \\
\hline \multirow{2}{*}{ capsid } & $\begin{array}{l}\text { ATCGA CAACG ACGTT CTGGC CGCGC TTAAG ACGAA GAAAG CATCC AAATA CGATC } \\
\text { TTGAG TATGC AGATG TGCCA CAGAA CATGC GGGCC GATAC ATTCA }\end{array}$ \\
\hline \multirow{2}{*}{ E1 } & $\begin{array}{l}\text { CACCA GGGTG TCAGA AACAC CGACA CTTTC AGCGG CCGAA TGCAC TCTTA ACGAG } \\
\text { TGCGT GTATT CTTCC GACTT TGGTG GGATC GCCAC GGTCA AGTAC }\end{array}$ \\
\hline \multirow{2}{*}{ E2 } & $\begin{array}{l}\text { CTTAA AAGGA AAACT GCATG TCCCA TTCTT GCTGG CAGAC GGCAA ATGCA CCGTG } \\
\text { CCTCT AGCAC CAGAA CCTAT GATAA CCTTC GGTTT CAGAT CAGTG }\end{array}$ \\
\hline \multirow{2}{*}{ nsP3 } & $\begin{array}{l}\text { CCACC ATGTG TCTGC TCGCC AATGT GACGT TCCCA TGTGC TCAAC CACCA ATTTG } \\
\text { AATGC TCCCA GCCTA TATTG TTCTC ACCGA AAGTG CCTGC GTATA }\end{array}$ \\
\hline \multirow{2}{*}{ nsP4 } & $\begin{array}{l}\text { GCTGG TTAGG AGATT AAATG CGGTC CTGCT TCCGA ACATT CATAC ACTGT TTGAT } \\
\text { ATGTC GGCTG AAGAC TTTGA CGCTA TTATA GCCGA GCACT TCCAG }\end{array}$ \\
\hline
\end{tabular}

purified with an RNeasy mini kit (Qiagen \#74104.) Purified RNA was quantified on a NanoDrop 2000 spectrophotometer (ThermoFisher, Wilmington, DE, USA) Samples were prepared for sequencing by rRNA depletion using the NEBNext rRNA Depletion Kit (\#E6310) followed by library prep using NEBNEXT Ultra II RNA Library Prep with Sample Purification Beads (\#E7775, NEB, Ipswich, MA, USA.) Libraries were quality checked on an Agilent BioAnalyzer before sequencing on an Illumina NextSeq 500 with a NextSeq 500/550 High Output Kit v2.5 (75 Cycles) kit (\#20024906, Illumina, San Diego, CA, USA.) 
The sequencing files were imported into Galaxy [325], trimmed using trimmomatic version 0.36.6 [326], aligned to viral reference sequences with BowTie 2 version 2.3.4.2 [327], and a pile-up was generated, filtered and exported to Excel for statistical analysis. Sequences not aligning with the viral references were aligned with the mouse mm10 genome (GenBank assembly accession GCA_000001635.8) using HiSat2 version 2.1.0+galaxy4 [328]. Mouse transcripts were quantified with htseq-count version 0.9.1 [329] and screened for differential gene expression by DESeq2 version 2.11.40.2 [330]. Gene ontologies relating to differential gene expression were detected by goseq mapping software version 1.26.0 [331].

\subsection{Statistical Analysis}

Results showing weights, clinical scores, or viral plaque assay titers are presented as mean \pm SEM using the GraphPad Prism version 7 for Windows package (GraphPad Software, LaJolla, CA, USA). Prism was also used for statistical significance testing, as indicated in the applicable figure legends, and to visualize data from NanoString and RNA-Seq experiments as heat-maps.

NanoString nSolver software (NanoString Technologies, Seattle, WA, USA) was used to generate all presented volcano plots and the data presented in supplemental Table 1 and 3. nSolver also calculated relative cell marker expression. Differences in cell marker expression were tested for significance in Prism by two-way ANOVA followed by Fisher's LSD post-hoc analysis.

NanoString nSolver Advanced Analysis 2.0 software was used to calculate pathway scores. Pathway scores are calculated based on the first principal component of each measured gene in the KEGG pathway. These differences are then quantified based on the normalized expression of all genes belonging to the pathway. This summarizes the involvement of each tested KEGG pathway for each biological replicate relative to all samples included in the analysis. Pathway 
scores summarize whether the overall gene expression in a pathway is up- or down-regulated. Values for pathway score were exported from nSolver, normalized to mock infected controls, and tested for significance in GraphPad Prism using a two-way ANOVA with Dunnett's post-hoc comparison to the mock IC treated group.

NanoString nSolver Advanced Analysis 2.0 software also calculates a "Global Significance Score" (GSS) for a group of samples by measuring the overall differential expression of all genes belonging to a particular KEGG pathway, while ignoring whether each gene is up- or downregulated. GSS is different from pathway score because it summarizes how much difference there is in pathway involvement between groups. GSS allows for the easier comparison of many pathways between groups, but does not provide meaningful data about the magnitude of overall gene expression.

\subsection{Ethics Statement}

All animal experiments were conducted under protocols approved by the University of Louisville institutional animal care and use committee. Work with live VEEV was conducted at the National Institutes of Health Regional Biocontainment Laboratory on the University of Louisville campus and was done with University of Louisville institutional biosafety committee approval. TC-83 and V4020 were handled with BSL2/ABSL2 practices and are exempt from US federal select agent regulations. Passaging of V4020 and TC-83 in mouse brains was done at ABSL3.

\section{Results}

\subsection{Kinetics Study: Attenuated Replication of V4020 in Brains of Infected Mice}


To assess replication kinetics in brain tissues, two groups of BALB/c mice were inoculated with $1 \times 10^{5} \mathrm{PFU}$ of either V4020 or TC-83 via the IC route. At 1-2 day intervals, three mice from each group were euthanized and brain samples were taken to determine infectious viral load by plaque titration. Mice from both experimental groups showed symptoms of disease and lost weight (Figure 14b), while a control group of mock-infected mice did not show clinical scores during the observation period (Figure 19). We previously documented that higher clinical scores correlated with weight loss in this model [44]. Direct comparison of percent weight loss to viral load reveals key differences between V4020 and TC-83 at 7 DPI (Figure 14b). Replication kinetics of both attenuated viruses showed similar patters during the first $3 \mathrm{DPI}$, reaching peak titers of $10^{9} \mathrm{PFU}$ per gram of brain tissue at $2 \mathrm{DPI}$. After $3 \mathrm{DPI}, \mathrm{V} 4020$ replication rapidly declined, with a significant decrease in viral titer at $5 \mathrm{DPI}$. No infectious V4020 viruses were detected at $7 \mathrm{DPI}$ or at later time points. Conversely, infectious TC-83 was detected at 7 DPI and 9 DPI (with titers of $10^{5}$ and $10^{4} \mathrm{PFU} / \mathrm{g}$ respectively). The high viral loads in TC-83-infected mice seemed to drive more aggressive weight loss than was observed in V4020-inoculated mice (Figure 14b).

In line with infectious viral load results, RNAScope in situ hybridization detected strong and extensive signals in the brain tissues of both groups of mice at early stage of the infection (Figure 15, 2 DPI, brown staining). In V4020-infected mice, these signals declined at 5 DPI and were not detectable at later time points. In contrast, in TC-83-infected mice, strong positive hybridization signals remained in brain tissues of TC-83 mice until 9 DPI and were still detectable as late as 13 DPI (Figure 15). Histology (H\&E staining) did not reveal clear differences between two groups of mice. Nevertheless, in TC-83-infected mice at 5 DPI, there was a notable increase in perivascular cuffing, a key indicator of VEEV brain inflammation (Figure 15, H\&E panels, arrowed). In summary, the replication kinetics study documented a more attenuated profile of VEEV V4020 replication in comparison with the VEEV TC-83 experimental vaccine. 


\subsection{Neuro-Inflammation in Brain Tissues of Mice Infected with V4020 vs. TC-83 Assessed by Direct Multiplexed Measurement of Gene Expression}

NanoString nCounter gene expression technology allows direct measurement of mRNA expression levels in tissues without enzymatic reactions [332]. This technology is more sensitive than DNA microarrays and comparable to real-time PCR measurements for individual genes $[333,334]$. In this study, the commercially available Mouse Neuro-Inflammation color-coded probe panel [335], enabling the quantification of 770 genes involved in 23 KEGG-pathways and processes, was used to assess host cell responses in brain tissues of V4020- and TC-83-infected mice. In addition, customized barcoded VEEV-derived probes were designed and included in this panel to detect and quantify the expression of individual VEEV genes.

Pathway scoring is a summary statistic that reflects the relative KEGG pathway involvement based on gene expression. As seen in Figure 16, inflammatory and cytokine signaling, as well as innate and adaptive immune response pathways, were significantly upregulated in both TC-83 and V4020-inoculated mice compared to mock-inoculated controls at 5 and 7 DPI. However, the magnitude of upregulation is much greater for TC-83 compared to V4020, and remains significantly higher than mock-infected mice at 9 and 13 DPI. Conversely, in V4020-inoclulated mice, pathways scores declined and are no longer statistically different from mock treated animals by 9 DPI (Figure 16a). In general, pathway scores peak at 5 DPI for VEEV V4020 and 9 DPI for VEEV TC-83 (Figure 16a) which corresponds to the last day that infectious viral particles are detected by plaque assay in brain tissue (Figure 14b).

Volcano plots provide visualization of the magnitude of differential gene (host and viral) expression in V4020 treated mice compared to TC-83 (X axis, log2[fold change]), along with statistical significance (Y axis, -log10[P-value]). There were no significant differences in gene expression patterns at 5DPI (Figure 20a). At 7, 9 and 13 DPI, VEEV viral genes were 
downregulated in V4020-inoculated mice compared to TC-83-inoculated animals (Figure 16b-d, named viral genes, red $[p<0.01]$ and green $[p<0.001]$ colored genes). At $9 \mathrm{DPI}$, the greatest number of genes displayed large log2-fold changes, and the changes were highly statistically significant (Figure 16c). Among these, host genes related to inflammation (labeled by orange boxes) were clearly identified. These inflammation related genes were less abundant at 13 DPI (Figure 16d). All differentially expressed genes, with a greater than $2 \log 2$-fold change in at least one passage, are listed in Table S1. VEEV viral genes E1, 6K, nsP4, nsP3, E2, capsid protein and E3 showed the greatest average magnitude of change, followed by host genes related to inflammation, including interleukin 1 receptor antagonist (Il1rn), granzyme B (Gzmb), macrophage scavenger receptor 1 (Msr1), leukocyte immunoglobulin like receptor B4 (Lilrb4a), granzyme A (Gzma) and chemokine genes (Ccl2, Cxcl10, ccl7, Cxcl9).

A heat-map of GSS scores showed clearly distinguished patterns between V4020 and TC-83 samples (Figure 20b). The GSS scores for mock and V4020 inoculated mice at 9DPI were very similar across all pathways, suggesting the processes involved in neuro-inflammation, damage, repair and adaptive immune responses have returned to baseline values at this time point. Meanwhile, "inflammatory signaling" and "neuron and neurotransmission" GSS had markedly different patterns between V4020 and TC-83 groups (Figure 20,c,d, respectively).

NanoString technology can also quantitate predicted cell type abundance based on mRNA expression probes specific for cell type markers presented in the Mouse Neuro-inflammation panel. Markers of macrophages, DCs, and microglia were expressed with statistically significant differences in brain tissues of V4020 mice in comparison with TC-83-inoculated mice. As seen in Figure 16e, in brain tissues of TC-83-inoculated mice, the expression of DC markers was strongly upregulated at 5, 7, and 9 DPI. Macrophage cell markers were generally upregulated in TC-83 compared to V4020, with statistically significant differences at 5 and $13 \mathrm{DPI}$. In addition to 
macrophages, markers of microglia were also clearly upregulated in TC-83-inoculated mice. In summary, direct multiplexed measurement of genes involved in mouse neuro-inflammation pathways clearly demonstrated upregulated patterns of genes involved in neuro-inflammation in mice inoculated with VEEV TC-83 versus VEEV V4020.

\subsection{Clinical Profiling of Mice during Serial Intracranial Passages of V4020 and TC-83}

Serial IC passages in mice are widely used to assess the phenotypic and genetic stability of alphavirus attenuation. This approach was used to compare the V4020 and TC-83 experimental vaccines. As seen in Figure 17, five sequential IC passages (P1-P5) of V4020 and TC-83 in BALB/C mice resulted in striking differences in clinical profiles. First, throughout the five serial passages, all V4020 treated mice survived, while three out of eight TC-83 mice met euthanasia criteria in the groups which received passage 2 (P2) and P3 virus (Figure 17a-b). Second, measurement of viral load at 2 DPI in brain tissues of mice revealed lower titers during P1-P3 in V4020 mice, and these differences were statistically significant. (Figure 17c). Third, mice infected with V4020 isolated from previous brain passages tended to have weight loss predominantly during the first $3 \mathrm{DPI}$, then gained weight after this time point. Some mice (e.g., in P1- and P2-V4020 groups) nearly fully recovered their initial weight by the end of observation period (Figure 17d). In contrast, mice in TC-83 groups tended to have a prolonged, more profound, weight loss. No mice in TC-83 groups recovered their initial weight (Figure 17e). Finally, clinical scoring of mice revealed that mouse-brain-passaged V4020 had clinical manifestations peaking at 2-3 DPI. After this time point, clinical signs were ameliorated in all mice, and, after 7-8 DPI, no V4020 animals had scored clinical symptoms (Figure 17f). In great contrast, mouse brain passaged TC-83 caused more severe clinical scores that persisted in some mice at 14 DPI (Figure 17g). Interestingly, clinical symptoms of TC-83 inoculated animals displayed a biphasic pattern (e.g., P3 and P4 TC- 
83, Figure 17g). These results indicate that IC passages of the VEEV V4020 experimental vaccine did not result in a change in the pathogenic profile from the initial V4020 inoculation (P1). In contrast, VEEV TC-83 passages resulted in the generation of viruses which caused aggravated experimental disease, which resulted in the euthanasia of $13.3 \%$ of TC-83 treated mice.

\subsection{Viral and Host RNA-Seq Profiles of Brain Samples, P1-P5}

During serial passaging in brain, RNA samples from tissue samples collected at 2 DPI were analyzed by Illumina NextSeq 500 to assess genetic variations in V4020 and TC-83 viral populations. In general, as seen in Figure 18a, the V4020 virus had lower rates of mutations that had high statistical significance throughout passages P1-P5 (downward spikes in blue for V4020 or grey for TC-83). Read depth among sequenced samples depicted in red for V4020 or purple for TC-83 was similar (Figure 18a). At 2 DPI, neither virus showed signs of selective pressure driving the reversion of either of the key attenuating mutations: the 5'UTR position 3-A or the E2-120 codon (Figure 18b). The rates of transversion and transition mutations were similar among all samples (Figure 21a,b). Synonymous to non-synonymous mutation ratios (SMR) were significantly lower for nsP1 and nsP2 genes and elevated for nsP3 genes in samples derived from both viruses (Figure 18c). The SMR ratio tended to increase for highly statistically significant mutations (Figure 21c). Interestingly, a significantly higher SMR was observed in the nsP4 (RdRP) gene of TC-83 compared to V4020. In particular, many more nucleotide variants (NVs) were detected in nsP3 and nsP4 of passage 2 and 3 of TC-83 than V4020, with several of these persisting through passages 4 and 5. Fewer NVs were observed in the capsid gene of V4020, with the largest differences in P2, P3, and P4.

Sequence reads mapping to the murine host allowed a basic analysis of the host response at 2DPI for V4020 compared to TC-83 infected mice. DESeq2 analysis with two factors, virus 
(V4020 or TC-83) and passage number, revealed 21 differentially expressed host genes.

However, none of these appear to segregate in a clear way to inoculation with V4020 or TC-83

(Figure 22a). A full list of differentially expressed host genes generated by DESeq2 in both the two factor analysis and, for (one factor, virus,) of each passage is presented in Table S2. Based on RNA-Seq at $2 \mathrm{DPI}$ and NanoString analysis from 5-13 DPI, 97 host genes were identified to be differentially expressed between V4020, and TC-83 in both assays (Table S3). Interestingly, among these genes-immune regulators Tbx21 (a key regulator of cytotoxic T-cell response) and Eomes, complement genes $\mathrm{C} 1 \mathrm{qa}$ and $\mathrm{C} 1 \mathrm{qb}$, the cytokine $\mathrm{Cx} 3 \mathrm{cl} 1$, and the lysosomal marker Lamp2-were identified as being differentially expressed in relation to both virus and time point (Table S3, fold changes in V4020 compared to TC-83). Ontological analysis of differentially expressed genes compiled from each individual passage highlighted that the pathways most likely to be differently regulated in V4020 compared to TC-83 IC-inoculated mice at 2 DPI predominantly involve neurogenesis and brain function (Figure 22b). In summary, RNA-Seq profiling of samples collected at 2 DPI during serial passages revealed lower rates of viral mutations in V4020 samples, and different patterns of host gene expression associated with neurogenesis and brain function in V4020- and TC-83-inoculated mice.

\section{Discussion}

Historically, live attenuated vaccines against viral infections have been among the safest and most efficacious medical interventions, and they continue to be the most cost-effective preventive measures $[1,336]$. In recent years, live attenuated vaccines against influenza (FluMist), rotavirus (Rotarix) and herpes (Zostavax) infections were licensed for human use. This indicates that the powerful tools of molecular virology which are currently available can be successfully applied to address the inherent weaknesses of these vaccines: genetic stability and 
reversion to wild-type phenotypes [337]. The TC-83 vaccine for VEEV was developed almost 60 years ago using the "classical" technique of multiple passages in vitro and in vivo, which resulted in two attenuating mutations, the first in the $5^{\prime}$-end untranslated region, position $3(G>A)$ and the second in the E2 envelope glycoprotein, position 120 (Thr > Arg) [13]. Since the 1960s, despite numerous attempts to develop better vaccines, TC-83 is the only vaccine in human use under an IND protocol (Special Immunization Program) which has allowed it to be tested in Phase II trial (NCT03531242, NCT00582504, NCT00582088, NCT03051386, https://ClinicalTrials.gov). Nevertheless, TC-83 is reactogenic and poorly immunogenic in 18\%$20 \%$ of vaccinees, which raises serious safety concerns.

The small number of attenuating mutations seems be associated with the genetic instability and reactogenicity of TC-83. To address this issue, we have applied several approaches to develop an advanced VEEV vaccine, V4020 [44], including: (i) rearrangement of structural genes, (ii) introduction of an additional sub-genomic promoter (26S) downstream from the glycoprotein genes; (iii) synonymous translational codon replacement to secure the attenuating E2 mutation TC-83; and (iv) the rescue of V4020 from a molecular clone (cDNA) to limit the population heterogeneity of the V4020. Here, we compared the replication kinetics of V4020 and TC-83 in the brain following IC inoculation of mice, and performed five IC mouse passages, using brain tissues as the source of the virus, to assess the genetic stability and heterogeneity of these experimental vaccines.

It is cannot be excluded that incomplete attenuation, characterized by the emergence of viruses with reversions, is responsible for the pathogenic features and adverse effects of TC-83 vaccination [303]. Notably, viruses with reversion mutations have been isolated from throat swabs of individuals vaccinated with TC-83 (Jahrling PB, unpublished data) [36]. Antiviral host responses following inoculation, along with the delay in the virus reaching the CNS, probably 
have synergistic effects on the attenuation of neuro-virulence. IC inoculation of experimental mice is a useful tool for short-cutting the natural infection route and directly examining the neuro-virulence of live attenuated vaccines.

We have shown here that IC inoculation of VEEV V4020 resulted in a more attenuated pattern of replication compared with TC-83, as determined by clinical manifestations (weight loss) and viral load in brain tissues of BALB/c mice (Figure 14b). The most striking difference between two infections was more than $2 \log _{10}$ differences in infectious viral load (PFU/g) in the brain at 5-7 days after IC inoculation. This was in line with RNAScope in situ hybridization data (Figure 15). High viral load seemed to be the major driving force, resulting in a spike in weight loss (which has previously been shown to correlate with clinical score [44]) at this time point in TC-83-infected mice.

$\mathrm{C} 3 \mathrm{H} / \mathrm{HeN}$ mice are considered a more susceptible VEE model in comparison to BALB/c mice [338]. However, IC inoculation of BALB/c with wild-type VEEV TrD and C3H/HeN mice with wildtype VEEV TrD or with VEEV TC-83 resulted in the same LD50 value, around 20 PFU [36]. In contrast, the experimental VEEV V3526 vaccine was not lethal in $\mathrm{C} 3 \mathrm{H} / \mathrm{HeN}$ mice. However, the poor safety profile of this vaccine in Phase I trial prevented further development [32]. The predominant adverse effects of VEEV TC-83 tend to be fever and headache [315], which coincide with the key symptoms of natural human VEEV infection [339]. In non-human primate models, central nervous system (CNS) invasion by TC-83 causes perivascular infiltration, gliosis, and signs of neurological disease [35]. IC inoculation of BALB/c mice recapitulates key features of neurovirulence following TC-83 vaccination including perivascular cuffing (Figure 15), monocyte accumulation in the CNS (Figure 16e,) and clinical signs of infection (Figure 14b, and Figure 17).

NanoString technology with a custom-made panel of barcoded probes for individual viral genes, combined with a commercially available mouse neuro-inflammation panel, allowed the 
direct detection and assessment of the involvement of viral and host genes in neuroinflammation of brain tissues in V4020- and TC-83-inoculated BALB/c mice. Volcano plots clearly illustrated differential expression of the genes in V4020 mice versus TC-83 mice at 5, 7 and 9 DPI, and (among host genes) genes related to inflammation pathways and processes prevailed in TC-83 mice (Figure 16, Table S1). In line with these results, markers of macrophages, DCs, and microglia were strongly upregulated in TC-83-infected mice, suggesting the involvement of these cells in inflammation. As further evidence that immune infiltration into the brain is responsible for weight loss and clinical manifestations (Figure 14), histology revealed perivascular inflammation foci in TC-83 brain H\&E-stained sections (Figure 15, H\&E staining, arrowed). Reversion to a neuro-virulent phenotype represents the greatest risk in using a live attenuated vaccine against VEEV [317]. It has been reported that attenuated strains of VEEV can revert to causing neuro-virulence in as few as three serial passages in mouse brain $[13,340]$. Indeed, in our experiments P3 and P4 TC-83 isolates induced clinically manifested infection and death. Interestingly enough, after serial passages in brain, TC-83 induced a biphasic disease (Figure 17g), a classical description of the murine model of wild-type VEEV [303]. In contrast, after five IC passages, V4020 did not induce lethal disease. Clinical manifestations were mild or moderate and disappeared in most animals within one week of IC inoculation. VEEV pathogenesis seems to be due to complex polygenic traits that are difficult to relate to NVs in the viral genome [304]. Unique NVs were not detected from more neuro-virulent passages of TC-83. However, TC-83 viruses from passages P2 and P3 had a high level of NVs which mapped within nsP4 versus V4020 viruses (Figure 18a). SMR, defined as the total number of base pair mutations without change in the amino acid code divided by the total number of base pair mutations, was used to detect purifying selection as the evolutionary mechanism of VEEV evolution [341]. For both TC-83 and V4020 experimental vaccines, which nearly completely 
share a sequence identity (except, as previously noted, for V4020 rearrangement and stabilizing E2 position 120 mutation), nsP1 and nsP2 had significantly reduced SMR over five serial passages in mouse brain, suggesting that mutation in these genes is likely deleterious. Conversely, E3 had an increased SMR. While the time was too short (2 DPI) to consider selective pressure and adaptive immunity involvement, it is interesting to note that E3 has been identified as a key target of humoral responses which are protective against VEEV-TrD [342]. It has been reported that TC-83 strains with lower-fidelity RdRP are more attenuated and immunogenic [343]. nsP4, the viral RdRP [304], had a higher SMR in TC-83 than V4020, however we did not detect differences in mutation frequency across the genome (Figure 21d), suggesting that no real difference in RdRP fidelity arose during passaging.

\section{Conclusions}

The lack of apparent differences in mutation frequency at key attenuating mutations, and the further lack of evidence for any positive selection during the time points studied, suggests that the strategy of stabilizing the E2 T120A is valid to decrease the appearance of revertants which would potentially arise by random mutation in the viral quasi-species at the peak of viral replication following vaccination. Structural rearrangement seems to additionally secure the attenuated phenotype of V4020. Taken together, these results indicate that V4020 is a safer and genetically more stable alternative to TC-83. Successful testing of V4020 in a non-human primate aerosol challenge model provides an additional argument for the clinical development of this experimental vaccine [323].

Supplementary Materials: The following are available online at https://doi.org/10.3390/vaccines8010114, Table S1: Differentially expressed genes detected by 
NanoString, Table S2: Differentially expressed genes for a "virus x passage" RNA-Seq model, Table S3: Differential expression of genes detected by both NanoString and RNA-Seq.

Supplemental Table Legends:

Supplemental Table 1. Mouse and viral genes that were differentially expressed with an adjusted $p$-value $<0.05$ and an absolute value of $\log 2(\mathrm{FC})>2$ from the NanoString $n$ Counter neuro-inflammation array with probes for viral genes added. Fold changes (FC) are reported as the change in V4020 expression compared to TC-83. Adjusted p-values were computed by nSolver using a model to compare individual samples by day of collection.

Supplemental Table 2. Mouse genes that were differentially expressed with an adjusted $p$-value $<0.05$ from RNA-Seq on 5 passages of V4020 and TC-83 in mouse brain as reported by DESeq2 model of virus $x$ passage or for comparison of samples in individual passages. FC are reported as the change in V4020 expression compared to TC-83. Adjusted p-values are based on the Benjamine-Hochberg correction.

Supplemental Table 3. Concatenation of gene fold change and significance from Table S1 and Table S2 for genes that were present in both NanoString and RNA-Seq analysis.

Author Contributions: Conceptualization, P.P. and I.S.L.; methodology, D.M.J. and I.S.L; software, D.M.J. and K.J.S.; validation, P.P. and I.S.L.; formal analysis, D.M.J.; investigation, D.M.J., J.D.J., T.L.P., Y.K.C., and R.S.A.; resources, D.C., I.T., P.P., and I.S.L.; data curation, D.M.J.; writing-original draft preparation, D.M.J.; writing-review and editing, D.M.J, K.J.S., D.C., I.T., P.P., and I.S.L.; visualization, D.M.J.; supervision, I.S.L.; project administration, D.M.J. and I.S.L.; funding acquisition, I.S.L. and P.P. All authors have read and agreed to the published version of the manuscript. 
Funding: Research reported in this publication was supported by the National Institute of Allergy and Infectious Diseases of the National Institutes of Health (NIH) under Award Number Al094863 (Peter Pushko, PI).

Acknowledgments: Sequencing and bioinformatics support for this work provided by NIH grants P20GM103436 (Nigel Cooper, PI) and P30GM106396 (Donald Miller, PI). We thank Ms. Jihye Kim for her technical support with the RNAScope assay. The contents of this work are solely the responsibility of the authors and do not represent the official views of the NIH or the National Institute for General Medical Sciences (NIGMS).

Conflicts of Interest: The authors declare no conflict of interest.

(a) Venezuelan Equine Encephalitis Virus strain Trinidad donkey
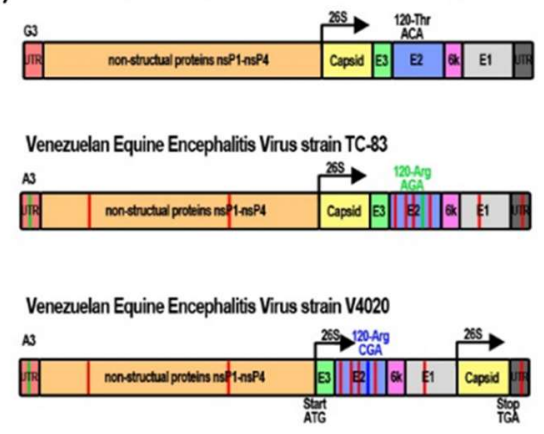

(b)

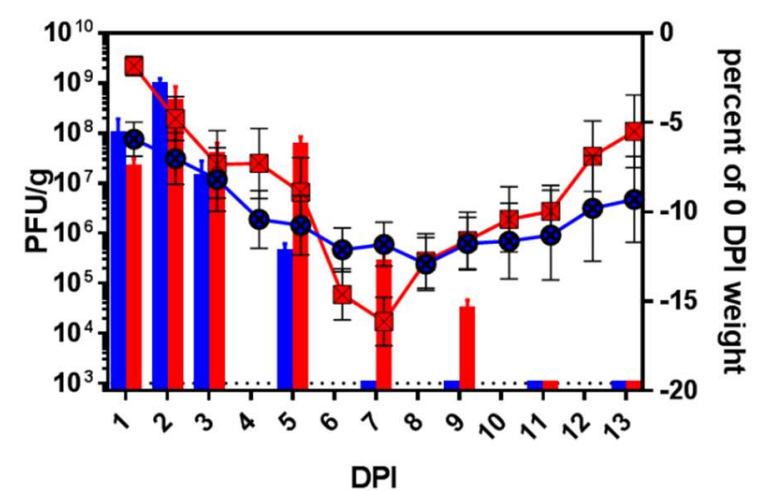

Figure 14. (a) Schematic diagram of the genomic structure of VEEV strains TrD, TC-83, and V4020. Red vertical lines denote nucleotide variants (NVs) with differing sequence from the TrD wild type strain which are not thought to play a key role in attenuation. Green vertical lines denote NVs with differing sequence between $\operatorname{TrD}$ and TC-83 which do play a key role in attenuation. A blue vertical line is used to denote the stabilized E2 120Arg NV in V4020. (b) Replication kinetics of V4020 and TC-83 in the brain of BALB/c mice ( $n=24$ per group) IC inoculated with $1 \mathrm{X} 10^{5} \mathrm{PFU}$ of either V4020 or TC-83 and monitored daily for 13 days. 
Weight loss (VEEV TC-83 in red square symbols and VEEV V4020 in blue circle symbols.) On days $1,2,3,5,7,9,11$, and 13 post inoculation, 3 mice per time point from each group were necropsied and brain tissue was prepared for VEEV infectious titration by plaque assay to assess viral load in brain tissues (VEEV TC-83 in red bars and VEEV V4020 in blue bars plotted against the left $\mathrm{Y}$ axis). 


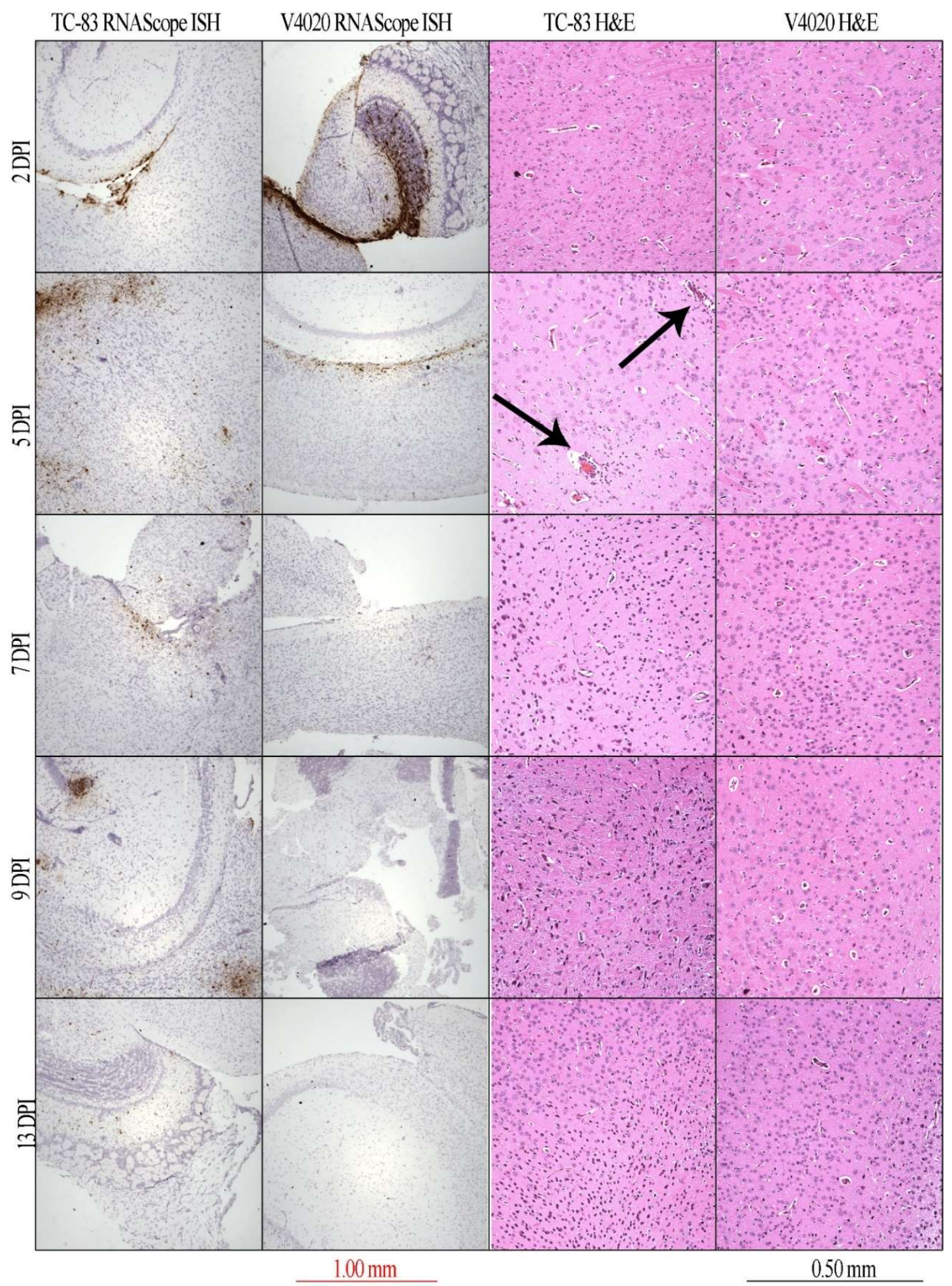

Figure 15. In situ hybridization by RNAScope and H\&E histology of brain sections. Stained tissue sections of V4020 and TC-83 brain tissue on day 2, 5, 7, 9, and 13-post IC inoculation. 
Brown staining in RNAScope images indicates the presence of viral RNA detected by an nsP3 in situ hybridization probe. Representative images at 50X magnification for RNAScope and 100X for H\&E are shown. Arrows are used to indicate perivascular cuffing.
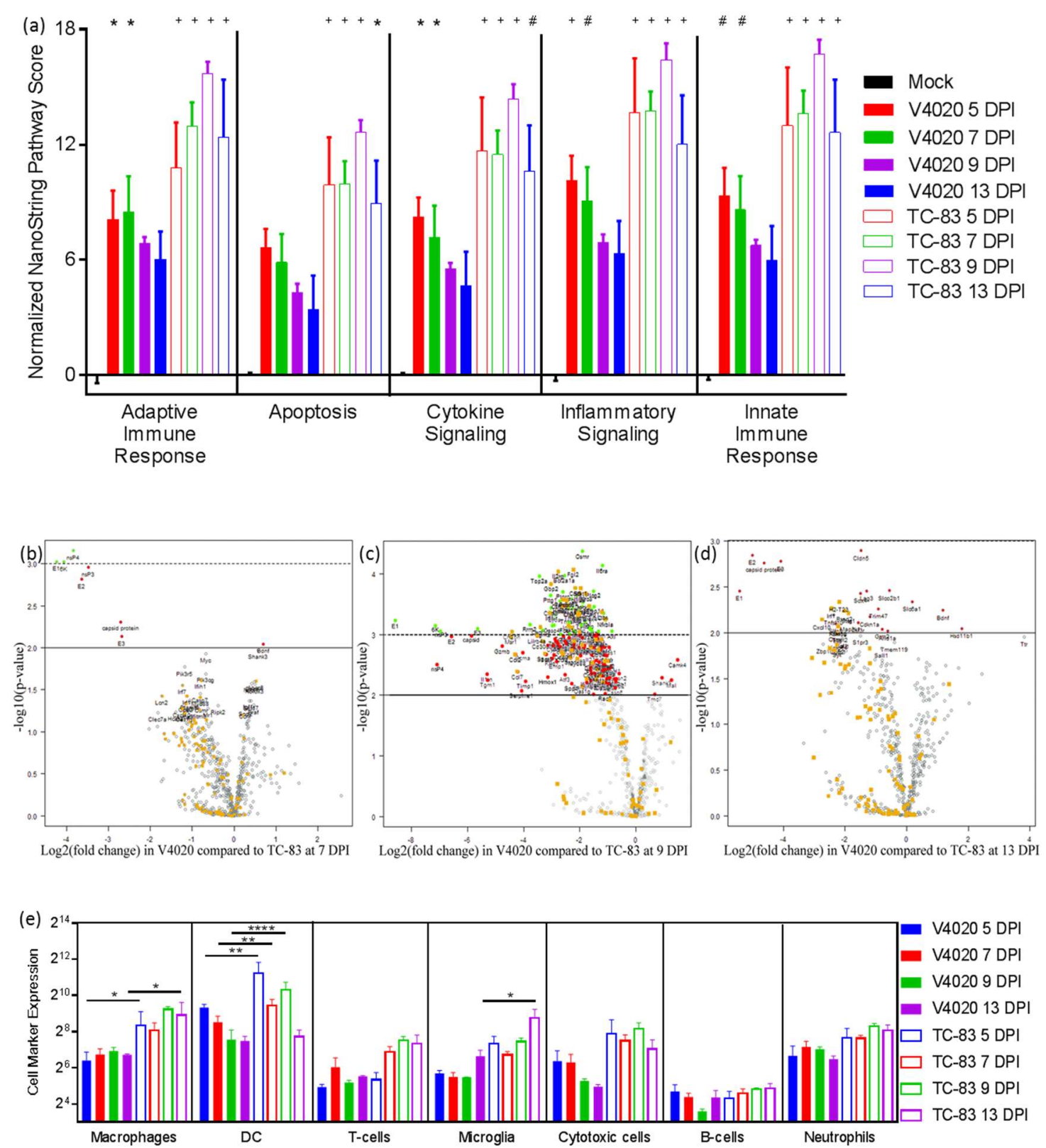

Figure 16. Detection and profiling genes involved in neuro-inflammation pathways. RNA was isolated from the brains of mice inoculated with V4020 or TC-83 and was quantified by NanoString nCounter assay using the mouse neuro-inflammation panel with additional 
probes to detect VEEV genes. (a) NanoString nSolver software was used to calculate a pathway score to summarize the differential expression data from all genes in the pathway based on the first principal component of normalized gene expression. Pathway scores were normalized to the average score for mock IC inoculation. Two-way ANOVA followed by multiple Student's t-tests with Dunnett's correction were used for post-hoc comparison to mock inoculated controls. ${ }^{*} p<0.01, \# p<.001,+p<0.0001$. Volcano plot presentation of differential gene expression in V4020-infected mice compared to TC-83 inoculated mice on (b) day 7, (c) day 9, and (d) day 13. Differentially expressed genes color coded according to adjusted significance where genes in red above the solid line represents the threshold of $p<0.01$ and genes in green are above the dashed line which represents $p<0.001$. Genes relating to the inflammatory KEGG pathway are labeled and shown in orange boxes (and are not additionally color coded by significance, but this can be determined by the lines on the graph). All significantly differentially expressed genes, (or the top 50 overall most significant genes for $7 \mathrm{DPI}$ ) have text names. (e) Cell type abundance in brain samples was determined by markers present in the NanoString mouse neuro-inflammation panel on days $5,7,9$, and 13 with significance determined for each time by a two-way ANOVA without matching followed by Fisher's LSD post-hoc analysis. ${ }^{*} p<0.05,{ }^{* *} p<0.01,{ }^{* * * *} p$ $<0.0001$. 
(a)

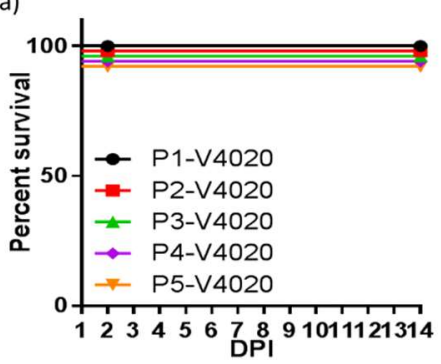

(d) $\rightarrow \mathrm{P} 1-\mathrm{V} 4020 \rightarrow \mathrm{P} 2-\mathrm{V} 4020 \rightarrow \mathrm{P} 3-\mathrm{V} 4020$

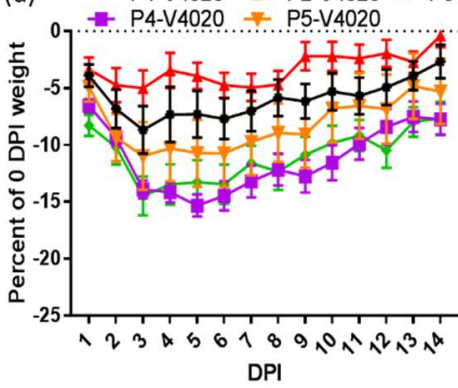

(b)
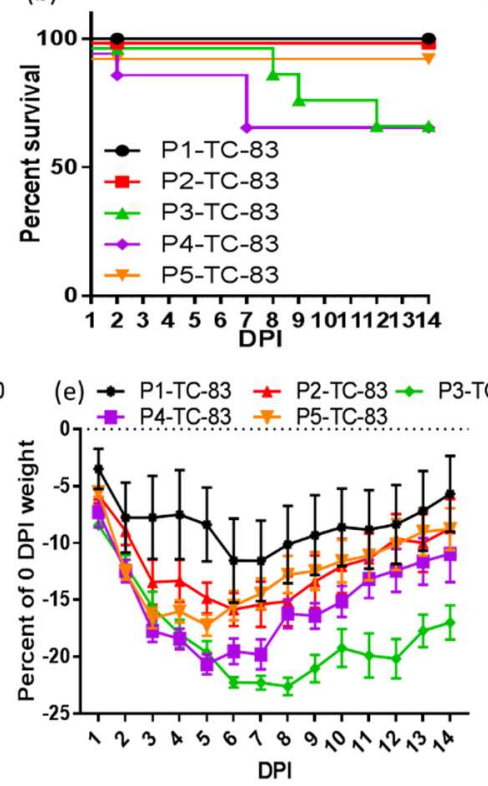

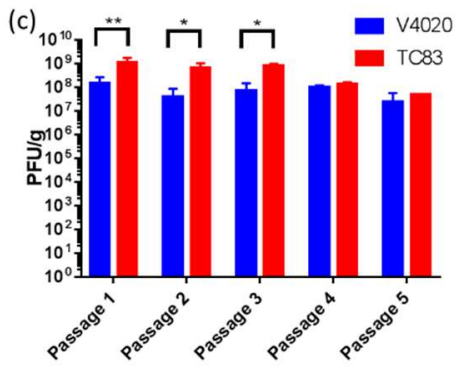

(f) ${ }^{5} \rightarrow=\stackrel{P}{P}-\mathrm{V}-\mathrm{V} 402020 \rightarrow \mathrm{P}_{\mathrm{P}-\mathrm{V} 4020} \rightarrow \mathrm{P}-\mathrm{V}-\mathrm{V} 4020$

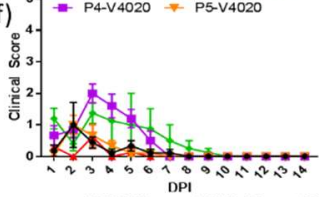

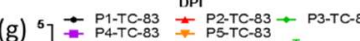

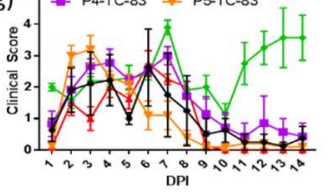

Figure 17. Groups of BALB/c mice $(n=10)$ were inoculated via the intracranial route with $1 \times 10^{5}$ PFU of either V4020 or TC-83. Two days after inoculation, two mice from each group were sacrificed, their brains were collected, homogenized, and used to make clarified viral stocks for inoculation of a subsequent group. This process was repeated four times for a total of five passages including the initial inoculation (named in the schematic of PX-Virus where $\mathrm{X}$ is the passage number and virus is either V4020 or TC-83.) Survival of (a) V4020 and (b) TC-83 passage groups was tracked during this time. (c) Brain titers from the mice sacrificed on day 2 post inoculation. Two-way ANOVA followed by Fisher's LSD post-hoc analysis were used to compare viral titers, ${ }^{*} p<0.05,{ }^{* *} p<0.01$. Daily weights (d) (e) and clinical scores (f) (g) were taken from the remaining mice challenged with either V4020 (d) (f) or TC-83 (e) (g) daily for 14 days. 

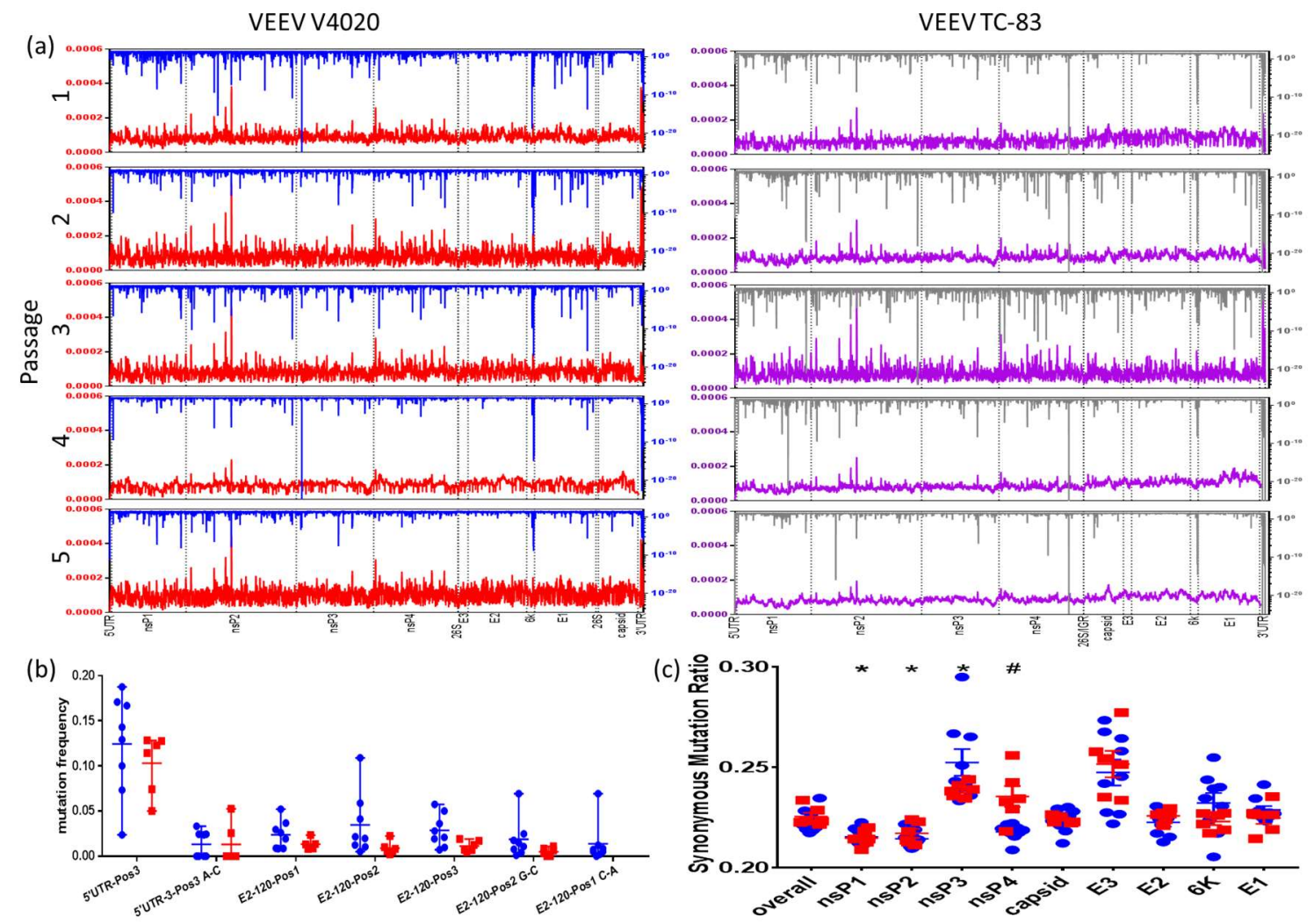

Figure 18. RNA-Seq by Illumina NextSeq was run on rRNA depleted RNA isolated from brain tissue of mice receiving passaged virus on day 2 post IC inoculation. Sequences were quality trimmed with trimmomatic and aligned to VEEV TC-83 or V4020 reference sequences with BowTie2. Pile-ups were generated and filtered in Galaxy before being exported to excel to calculate normalized read depth (a), statistical significance of mutations (a), mutation frequency (b) and SMR (c). (a) Normalized read depth plotted on the left y-axis (red for V4020, purple for TC-83) was calculated by dividing the number of virus-aligned reads at each position by the sum of the number of reads for every position. Statistical significance of mutation rate as a Z-score plotted by nucleotide position on the right y-axis (blue for V4020 and grey for TC-83) was calculated in Excel using the "NORM.DIST" function to calculate a probability density function based on the mean and standard deviation of each biological sample. The boundaries between genes and certain features are marked with a 
dashed vertical line and labeled at the bottom of the panel. Z-scores over $10^{-25}$ are reported as this value. Passages 2-5 of V4020 and Passage 1 of TC-83 are the average of 2 biological replicates. All other data are representative of a single biological replicate. (b) Mutation frequency calculated as a ratio of a mutation occurring at the identified location divided by the total number of reads for each reported location for V4020 (blue) and TC-83 (red). (c) SMR for the overall coding sequences and individual genes of V4020 (blue) and TC-83 (red). $* p<0.05$ for the indicated gene compared to the overall SMR by one-way ANOVA with Dunnettt's post-hoc test, \# $p<0.05$ for the SMR of nsP4 in V4020 compared to TC-83 by student's t-test. Data in this figure are averaged from 2 mice per group except for V4020 passage 1 and TC-83 passages 2 through 5 which represent a single mouse.
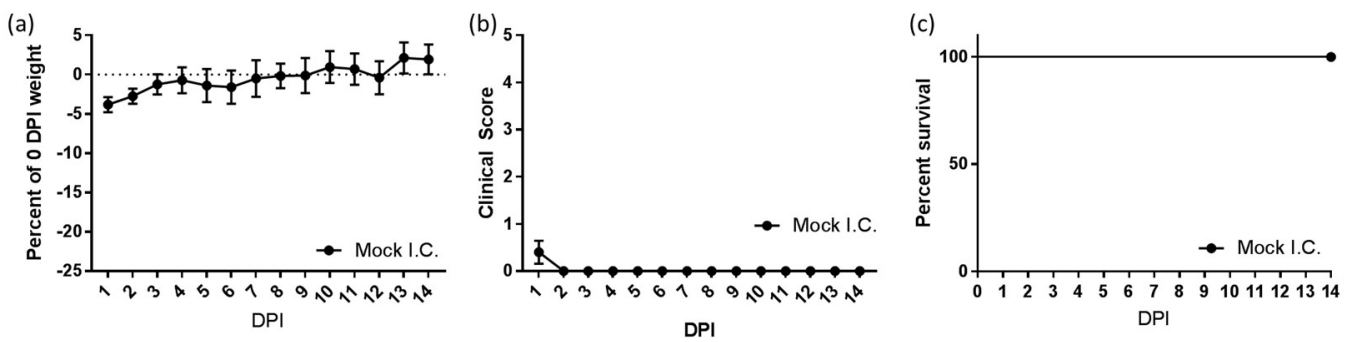

Figure 19. A group of BALB/c mice $(n=5)$ were inoculated IC with $10 \mu l$ of normal saline as a control for animals similarly inoculated with virus. These mice were followed for 14 days for (a) weight, (b) clinical score, and (c) survival. 
(a)

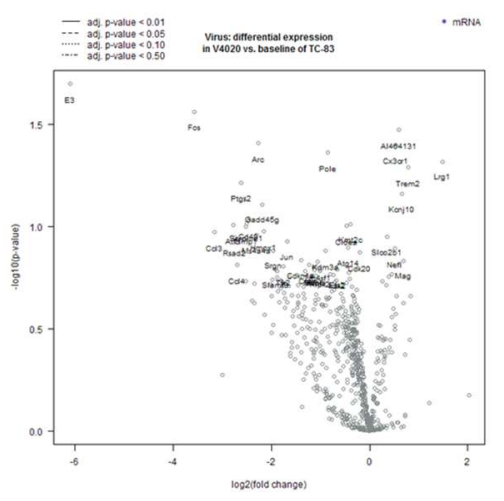

(b)

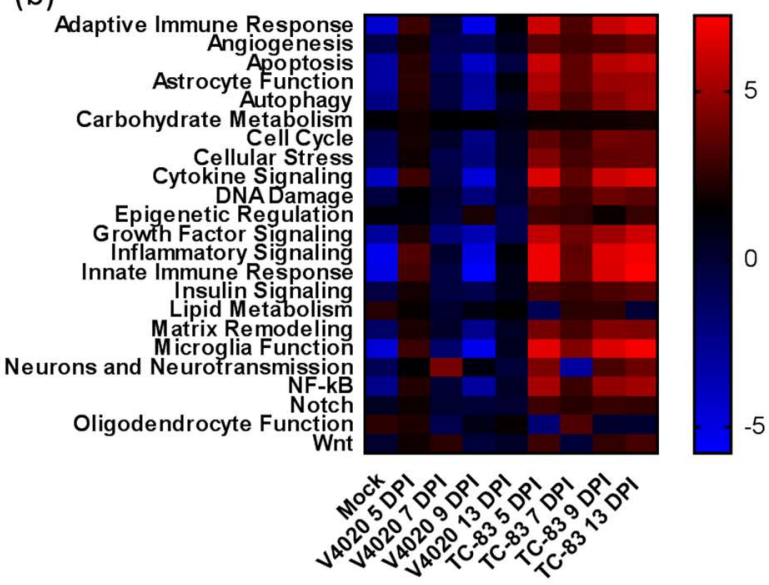

(c)

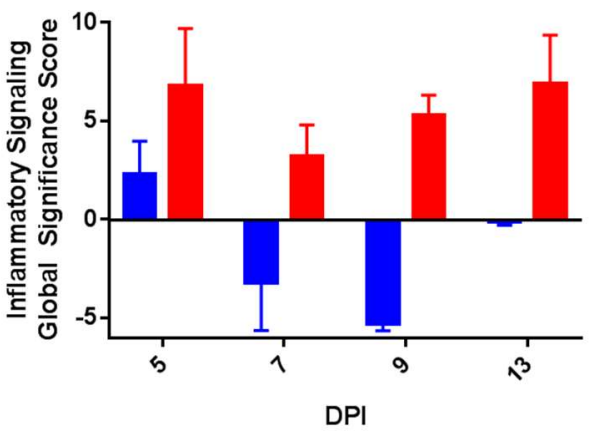

(d) o

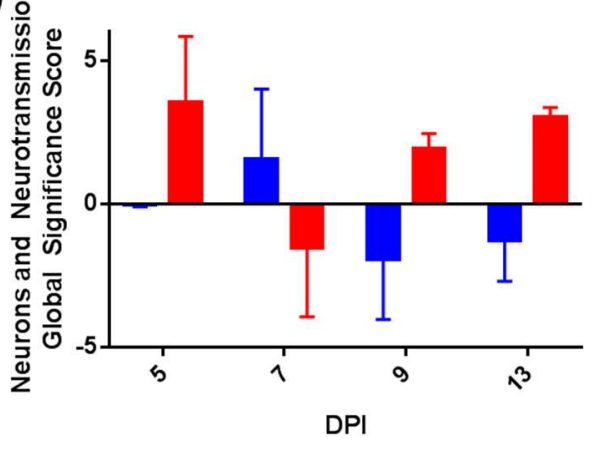

Figure 20. RNA was isolated from the brains of mice inoculated with V4020 or TC-83 as described in Figure 16 and was quantified by NanoString nCounter assay using the mouse neuro-inflammation panel with additional probes to detect VEEV RNA. (a) Differential gene expression in V4020 compared to TC-83 inoculated mice on day 5. (b) NanoString nSolver software was used to calculate a GSS (a measure of the overall differential expression of genes belonging to a particular KEGG pathway ignoring whether each gene is up- or downregulated) for each experimental grouping. Calculated NanoString GSS of the (c) inflammatory signaling and (d) neurons and neurotransmission gene sets for V4020 (blue) and TC-83 (red) on the indicated days. 
(a)

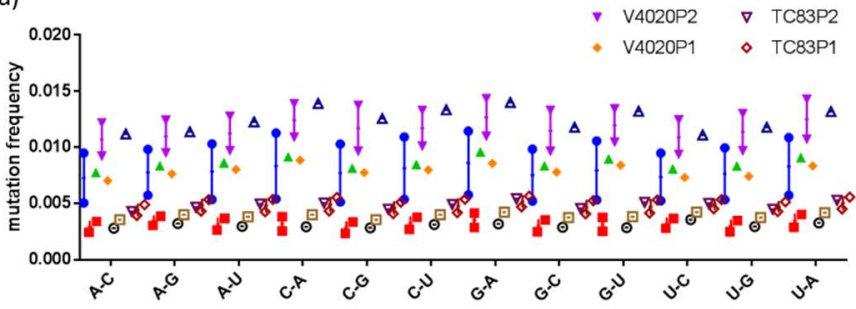

(b)

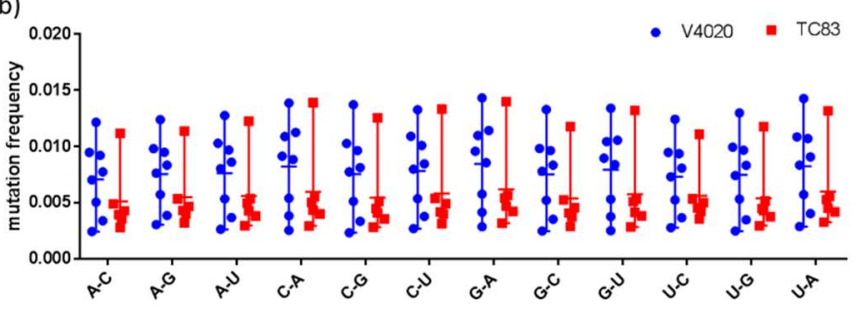

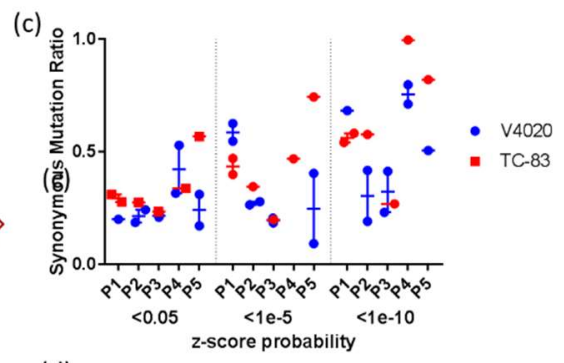

(d)

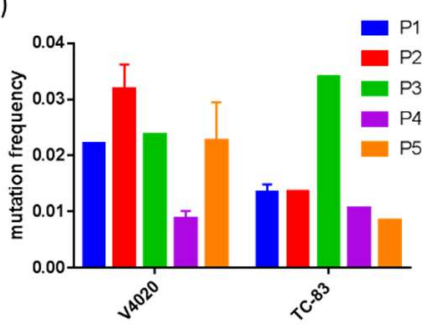

Figure 21. Frequency of transitions and transversions by base pair calculated as the counts of the specified mutation occurring divided by the total reads at all positions with the specified reference nucleotide grouped by (a) virus and passage or by (b) virus. (c) The SMR for all positions (overall) with a significance of Z-score less than the indicated value. (d) Mutation frequency by virus and passage for the whole virus genome from 2 DPI RNA-Seq calculated as the sum of the number of non-reference reads at every position divided by the sum of the total number of reads at every position. 
(a)

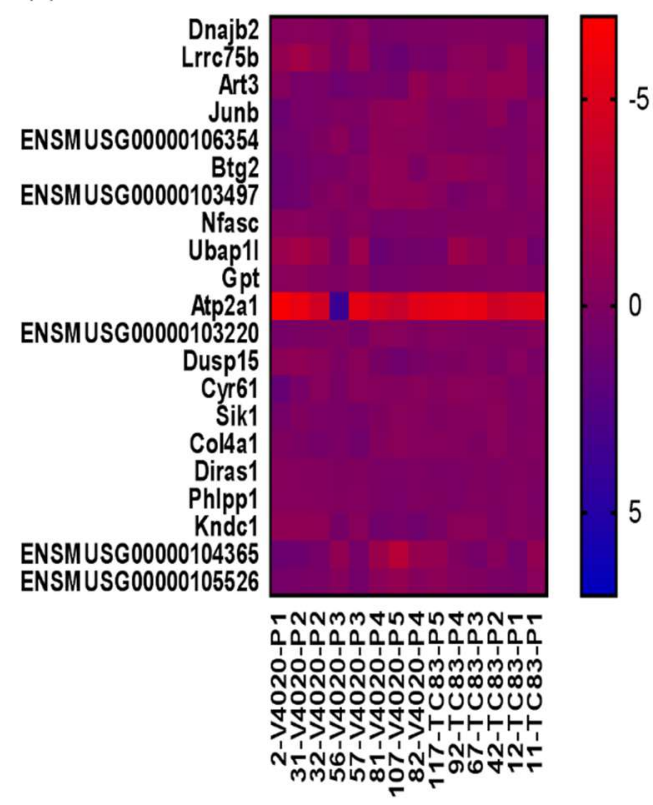

(b)

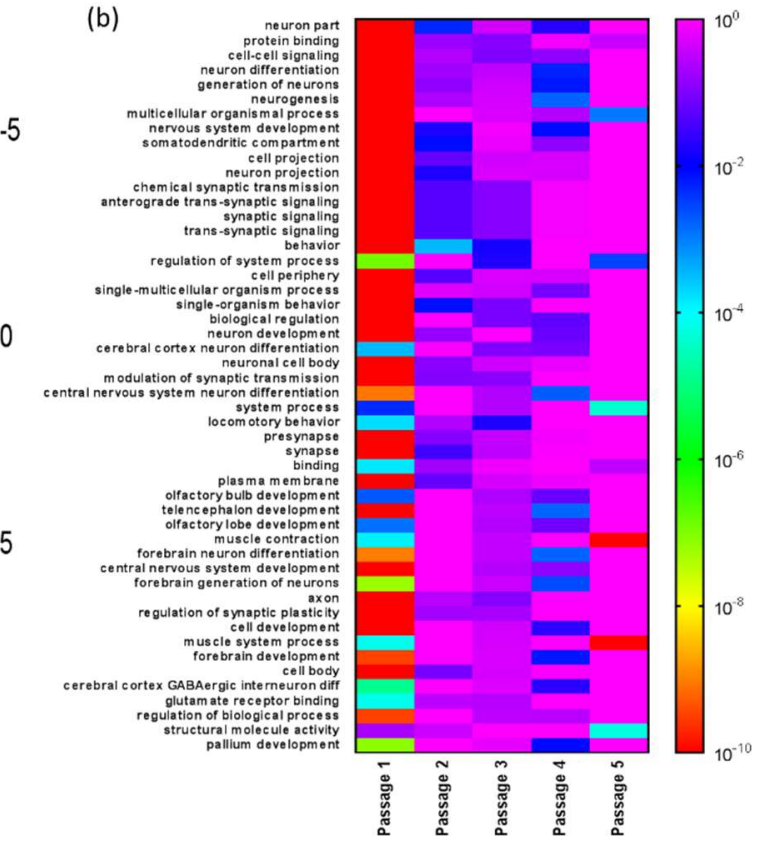

Figure 22. RNA-Seq data was generated and trimmed as described in Figure 18. All analysis described was performed using the named modules in the Galaxy web-based platform. Sequences were aligned to the mouse $\mathrm{mm} 10$ genome using HiSat2, and features were counted with htseq-count. (a) All genes with significant differential expression in a model accounting for both virus vaccine strain and passage number are presented in a heat map showing fold change. All genes with differential expression with an adjusted $p$-value greater than 0.05 from each individual passage that were detected by DeSEQ2 were used as the input to run GOseq on each passage individually. The pathways reported by GOseq for each passage were combined and sorted by significance. (b) The overall top 50 over-represented ontologies are displayed in a heat map depicting the associated adjusted p-value associated with the likelihood of over-representation from GOseq. p-values less than $10^{-10}$ are reported as $10^{-10}$. 


\section{CHAPTER VI}

\section{DISCUSSION AND CONCLUSIONS}

\section{Discussion}

Applications of arenavirus $L$ derived minigenome systems to study $L$-segment cis-acting factors

The L-segment based MG system will allow future studies to elucidate the role of cisacting factors (namely the $3^{\prime}$ untranslated region, the intergenic region (IGR), and the $5^{\prime}$ untranslated region) in interactions with the viral RdRP L and NP. This will facilitate the elucidation of the mechanisms responsible for the generation of the L-segment small RNAs that arise during ML29 replication (Figure 10). rLCMV containing a L segment where the IGR has been replaced with the S segment IGR is deeply attenuated [344]. We speculate that the mismatch of the ML29 (MOPV derived) L IGR with LASV NP protein may play a role in the generation of small RNAs observed. Additionally, further inquiry is needed to determine if the rescue of trisegmented arenaviruses with duplicated L-segments is possible. It is thought that the limiting factor for inclusion of segments in virions for arenavirus rescue is the overall size of the combined genome [345]. If a tri-segmented L based particle can be generated, it may additionally be possible to generate quad-segmented particles if the additional genes have a total length of no more than around $3 \mathrm{~kb}$.

While tri-segmented reporter expressing viruses are typically not as fit as their wild-type counterparts in vitro [283], they still may be a useful tool to study the generation of DIPs. In a proof of concept study, mice generated immunity to reporter sequences included in trisegmented ML29 viruses [346]. This suggests that despite differences in fitness, tri-segmented 
viruses could be used for in vivo imaging of reporter expressing genes to track the establishment of persistent infection. The L-segment MG system can also be used as the basis to study the generation of DIPs by providing different reporters expressed from the $Z$ and $L$ loci to track the relative expression of $Z$ and $L$ genes. If there were DIG competition for viral replicase complexes, this could be observed as a uniform decrease in the reporter signal from any of the promoter genes. Over expression of the Z protein from both LCMV and TCRV interferes with transcription and replication, a phenomenon known as $Z$ mediated superinfection exclusion [248, 347]. To exclude the possibility that there is an over production of matrix protein causing $\mathrm{Z}$ mediated superinfection exclusion, the ratio of $Z$ loci reporter to $L$ loci reporter would be observed.

Tri-segmented viruses may also be useful for the expression of additional antigens in arenavirus LAVs. For ML29, the additional gene segments could be used to include epitopes from diverse strains of LASV, or even other circulating viruses. LASV and EBOV co-circulate, and result in LF and Ebola virus disease (EVD) with similar symptoms [348]. The currently approved vaccine approaches for EBOV rely on LAV vectored vaccines [6], which leads us to hypothesize that a tri-segmented ML29 expressing EBOV antigenic determinants could be a useful vaccine with coverage against both African hemorrhagic fever viruses.

Rearrangement of structural genes is a useful tool to generate attenuated alphavirus vaccines

Genetic rearrangement of the structural genes of VEEV was demonstrated to be a useful tool to further attenuate alphavirus vaccine candidates. Partial transcription termination, where the viral RdRP will fall off of the template between genes leads to increased expression genes that are closer to the native $26 \mathrm{~S}$ sub-genomic promoter [349]. As a result, the rearrangement and addition of a second 265 sub-genomic promoter likely changes the ratio of capsid to glycoprotein produced in VEEV V4020 compared to VEEV TC-83. While clear differences in the 
viral gene expression between VEEV V4020 and VEEV TC-83 were observed (Figure 16), the mechanisms of attenuation that are attributable to the additional promoter remain unclear. However, the shift in the ratio of capsid to glycoprotein would likely decrease the efficacy of virion assembly. We hypothesize that this could be observed in differences in the ratio of particles (detected by qRT-PCR, which would also allow the detection of DIPs) to infectious units (detected by FFU or PFU). If this were the case, perhaps due to the contribution of DIPs, a higher particle to PFU ratio would be observed in VEEV V4020 compared to VEEV TC-83. Additionally, rearrangement of the structural genes could be a useful tool to generate other attenuated alphaviruses for use as LAVs, or to enhance the attenuation of other LAV candidates.

\section{Defining the pathogenic determinants of passaged VEEV infection}

Both VEEV TC-83 and VEEV V4020 replicated to high titer in the brain of IC challenged mice by $2 \mathrm{DPI}$. We made three key observations about the emergence of pathogenicity i) there are differences in virulence that emerge between passaged VEEV TC-83 and VEEV V4020, ii) neither the key attenuating mutations nor any of the other SNPs between VEEV TrD and VEEV TC-83 are reverted at $2 \mathrm{DPI}$, and iii) the emergence of pathogenic reversions seems to be a somewhat random event. Based on these finding, we speculate that the IC model allows for explosive expansion of the viral quasispecies before innate antiviral host responses can control the growth. During this time, there is relatively little selective pressure on the virus. Eventually host innate, and then adaptive, responses begin to cull mutants that most strongly elicit these responses.

The emergence of pathogenesis could be explained by a number of phenomena: i) either random or selective pressure could lead to the selection of reversions at the sites of VEEV TrD attenuation, ii) there may be a selection of mutants that evade host detection or elicit non- 
protective responses, or iii) there may be a selection of mutants that strongly engage host responses leading to pathogenesis by from overly vigorous inflammatory responses.

Additionally, it is interesting to consider whether pathogenesis observed in passaged VEEV TC83 IC challenge is due to a consistent defined set of mutations or a more random accumulation of minor pathogenesis contributors. Paradoxically, it has been noted that reduced fidelity of the viral replicase attenuates alphaviruses [350], however, we observed increased pathogenesis in VEEV TC-83 that seemed to have more genetic variability (Figure 17, 18).

Sequencing of viral RNA isolated from necropsy brain samples of mice that received passaged VEEV TC-83 and met early euthanasia criteria, would provide insight to the nature of the pathogenic revisions we observed. Based on the recovered sequences, future experiments to document the time course of infection with these mutants could be designed.

Additionally, many of these questions could be answered by set of passaging experiments with more passages from more individual donors that also incorporate serial sacrifice to collect RNA from the brain at multiple time points, followed by RNA-Seq analysis of the viral quasispecies and host responses. Presumably, there would be differences in host responses to either pathogenic or non-pathogenic passages of VEEV TC-83, especially at times after strong engagement of innate immunity. Perhaps more interesting is how much variation is there in the host responses to pathogenic passaged virus. As described here and previously, lethal viruses arose in $20 \%-40 \%$ of passages of VEEV-TC83 [36], but was not observed in the first two passages.

\section{Conclusions}

ML29 was attenuated compared to MOPV in STAT-1\% mice and did not show any signs of causing sensorineural hearing loss. Additionally, enhancing ML29 with the addition of DIPs 
increased protective T-cell responses in this model. ML29 was also immunogenic in Hartley outbred guinea pigs, and neither ML29 nor ML29P50 displayed signs of renal or hepatic toxicity in this model. ML29P50 was deeply attenuated compared to ML29 both in vitro and in the highly sensitive CBA/J IC challenge model. Furthermore, the newly described L-segment derived MG system will be useful to study the mechanisms of DIP interference. ML29 is a broadly protective and safe vaccine against LASV and is an ideal candidate for further clinical developments.

VEEV V4020 also an excellent candidate for clinical development. It is based on VEEV TC83 which has been used safely in human phase II trials, but further enhances safety. The mouse neuro-virulence data presented here, combined with the protective efficacy and favorable safety profile from the NHP study [323] support the application for US FDA investigational new drug status.

In conclusion, this work builds upon two LAVs, ML29 and VEEV 4020, which are both based on attenuation of their respective wild-type viruses. Both were demonstrated favorable safety and immunogenicity using advanced small animal models, which makes them both excellent candidates for further vaccine development. 


\section{REFERENCES}

1. Minor, P.D., Live attenuated vaccines: Historical successes and current challenges. Virology, 2015. 479-480: p. 379-92.

2. Chen, J.R., et al., Better influenza vaccines: an industry perspective. J Biomed Sci, 2020. 27(1): p. 33.

3. Warren-Gash, C., H. Forbes, and J. Breuer, Varicella and herpes zoster vaccine development: lessons learned. Expert Rev Vaccines, 2017. 16(12): p. 1191-1201.

4. Griffin, D.E., Measles Vaccine. Viral Immunol, 2018. 31(2): p. 86-95.

5. Sartorio, M.U.A., et al., Rotavirus vaccines in clinical development: Current pipeline and state-of-the-art. Pediatr Allergy Immunol, 2020. 31 Suppl 24: p. 58-60.

6. Bache, B.E., M.P. Grobusch, and S.T. Agnandji, Safety, immunogenicity and risk-benefit analysis of rVSV-DeltaG-ZEBOV-GP (V920) Ebola vaccine in Phase I-III clinical trials across regions. Future Microbiol, 2020.

7. Chen, L.H. and M.E. Wilson, Yellow fever control: current epidemiology and vaccination strategies. Trop Dis Travel Med Vaccines, 2020. 6: p. 1.

8. Ledgerwood, J.E., et al., Chimpanzee Adenovirus Vector Ebola Vaccine. N Engl J Med, 2017. 376(10): p. 928-938.

9. Rauch, S., et al., New Vaccine Technologies to Combat Outbreak Situations. Frontiers in immunology, 2018. 9: p. 1963-1963.

10. Ramezanpour, B., et al., Vector-based genetically modified vaccines: Exploiting Jenner's legacy. Vaccine, 2016. 34(50): p. 6436-6448.

11. Humphreys, I.R. and S. Sebastian, Novel viral vectors in infectious diseases. Immunology, 2018. 153(1): p. 1-9.

12. Tangy, F. and P. Desprès, Yellow Fever Vaccine Attenuation Revealed: Loss of Diversity. The Journal of Infectious Diseases, 2013. 209(3): p. 318-320.

13. Berge, T.O., I.S. Banks, and W.D. Tigertt, Attenuation of Venezuelan Equine Encephalomtelitis Virus by In Vitro Cultivation in Guinea-Pig Heart Cells. American Journal of Epidemiology, 1961. 73(2): p. 209-218.

14. Mateo, M., et al., Vaccines inducing immunity to Lassa virus glycoprotein and nucleoprotein protect macaques after a single shot. Sci Transl Med, 2019. 11(512).

15. Fisher-Hoch, S.P., et al., Effective vaccine for lassa fever. Journal of virology, 2000. 74(15): p. 6777-6783.

16. Lukashevich, I.S., et al., Safety, immunogenicity, and efficacy of the ML29 reassortant vaccine for Lassa fever in small non-human primates. Vaccine, 2008. 26(41): p. 5246-54.

17. Lukashevich, I.S., et al., A live attenuated vaccine for Lassa fever made by reassortment of Lassa and Mopeia viruses. Journal of virology, 2005. 79(22): p. 13934-13942.

18. Lukashevich, I., Generation of reassortants between African arenaviruses. Virology, 1992. 188(2): p. 600-605.

19. Weaver, S.C., et al., VENEZUELAN EQUINE ENCEPHALITIS. Annual Review of Entomology, 2003. 49(1): p. 141-174.

20. Walton, T.E. and M.A. Grayson, Venezuelan equine encephalomyelitis. The arboviruses: epidemiology and ecology, 1988. 4: p. 203-231.

21. Paessler, S., et al., Recombinant sindbis/Venezuelan equine encephalitis virus is highly attenuated and immunogenic. Journal of virology, 2003. 77(17): p. 9278-9286.

22. Rusnak, J.M., et al., Risk of occupationally acquired illnesses from biological threat agents in unvaccinated laboratory workers. Biosecurity and bioterrorism: biodefense strategy, practice, and science, 2004. 2(4): p. 281-293. 
23. Hawley, R.J. and E.M. Eitzen Jr, Biological Weapons-a Primer for Microbiologists1. Annual review of microbiology, 2001. 55.

24. Medina, G., et al., Genetic diversity of Venezuelan alphaviruses and circulation of a Venezuelan equine encephalitis virus subtype IAB strain during an interepizootic period. The American journal of tropical medicine and hygiene, 2015. 93(1): p. 7-10.

25. Weaver, S.C., et al., Genetic evidence for the origins of Venezuelan equine encephalitis virus subtype IAB outbreaks. Am J Trop Med Hyg, 1999. 60(3): p. 441-8.

26. Anishchenko, M., et al., Venezuelan encephalitis emergence mediated by a phylogenetically predicted viral mutation. Proceedings of the National Academy of Sciences, 2006. 103(13): p. 4994-4999.

27. Johnson, D.M., et al., Advanced Safety and Genetic Stability in Mice of a Novel DNALaunched Venezuelan Equine Encephalitis Virus Vaccine with Rearranged Structural Genes. Vaccines (Basel), 2020. 8(1).

28. Burke, D.S., H.H. Ramsburg, and R. Edelman, Persistence in humans of antibody to subtypes of Venezuelan equine encephalomyelitis (VEE) virus after immunization with attenuated (TC-83) VEE virus vaccine. Journal of Infectious Diseases, 1977. 136(3): p. 354-359.

29. Pittman, P.R., et al., Long-term duration of detectable neutralizing antibodies after administration of live-attenuated VEE vaccine and following booster vaccination with inactivated VEE vaccine. Vaccine, 1996. 14(4): p. 337-343.

30. Paessler, S. and S.C. Weaver, Vaccines for Venezuelan equine encephalitis. Vaccine, 2009. 27 Suppl 4: p. D80-D85.

31. Cole, F.E., Jr., S.W. May, and G.A. Eddy, Inactivated Venezuelan equine encephalomyelitis vaccine prepared from attenuated (TC-83 strain) virus. Appl Microbiol, 1974. 27(1): p. 150-3.

32. Martin, S.S., et al., Evaluation of formalin inactivated V3526 virus with adjuvant as a next generation vaccine candidate for Venezuelan equine encephalitis virus. Vaccine, 2010. 28(18): p. 3143-3151.

33. Davis, N.L., et al., Attenuated mutants of Venezuelan equine encephalitis virus containing lethal mutations in the PE2 cleavage signal combined with a second-site suppressor mutation in E1. Virology, 1995. 212(1): p. 102-10.

34. Fine, D.L., et al., Venezuelan equine encephalitis virus vaccine candidate (V3526) safety, immunogenicity and efficacy in horses. Vaccine, 2007. 25(10): p. 1868-76.

35. Fine, D.L., et al., Neurovirulence evaluation of Venezuelan equine encephalitis (VEE) vaccine candidate V3526 in nonhuman primates. Vaccine, 2008. 26(27-28): p. 3497-506.

36. Ludwig, G.V., et al., Comparative neurovirulence of attenuated and non-attenuated strains of Venezuelan equine encephalitis virus in mice. The American journal of tropical medicine and hygiene, 2001. 64(1): p. 49-55.

37. Paessler, S., et al., Replication and clearance of Venezuelan equine encephalitis virus from the brains of animals vaccinated with chimeric SIN/VEE viruses. J Virol, 2006. 80(6): p. 2784-96.

38. Roy, C.J., et al., A chimeric Sindbis-based vaccine protects cynomolgus macaques against a lethal aerosol challenge of eastern equine encephalitis virus. Vaccine, 2013. 31(11): p. 1464-1470.

39. Nasar, F., et al., Eilat virus host range restriction is present at multiple levels of the virus life cycle. Journal of virology, 2015. 89(2): p. 1404-1418. 
40. Nasar, F., et al., Eilat virus, a unique alphavirus with host range restricted to insects by RNA replication. Proceedings of the National Academy of Sciences of the United States of America, 2012. 109(36): p. 14622-14627.

41. Erasmus, J.H., et al., Novel Insect-Specific Eilat Virus-Based Chimeric Vaccine Candidates Provide Durable, Mono- and Multivalent, Single-Dose Protection against Lethal

Alphavirus Challenge. Journal of virology, 2018. 92(4): p. e01274-17.

42. Nasar, F., et al., Eilat virus induces both homologous and heterologous interference. Virology, 2015. 484: p. 51-58.

43. Tretyakova, I., et al., Novel vaccine against Venezuelan equine encephalitis combines advantages of DNA immunization and a live attenuated vaccine. Vaccine, 2013. 31(7): p. 1019-1025.

44. Tretyakova, I., et al., Novel DNA-launched Venezuelan equine encephalitis virus vaccine with rearranged genome. Vaccine, 2019. 37(25): p. 3317-3325.

45. Radoshitzky, S.R., et al., Past, present, and future of arenavirus taxonomy. Arch Virol, 2015. 160: p. 1851-1874.

46. Abudurexiti, A., et al., Taxonomy of the order BUNYAVIRALES: update 2019. Arch Virol, 2019: p. https://doi.org/10.1007/s00705-019-04253-6

47. Radoshitzky, S.R., et al., ICTV Virus Taxonomy Profile: Arenaviridae. J Gen Virol, 2019: p. in press.

48. Watanabe, Y., et al., Structure of the Lassa virus glycan shield provides a model for immunological resistance. Proceedings of the National Academy of Sciences, 2018.

49. Lukashevich, I.S. and M.S. Salvato, Lassa Virus Genome. Current Genetics, 2006. 7: p. 351-379.

50. Li, S., et al., Acidic $p H$-Induced Conformations and LAMP1 Binding of the Lassa Virus Glycoprotein Spike. PLoS Pathogens, 2016. 12(2): p. e1005418.

51. Hastie, K.M. and E.O. Saphire, Lassa virus glycoprotein: stopping a moving target. Current Opinion in Virology, 2018.

52. Ehichioya, D.U., et al., Current molecular epidemiology of Lassa virus in Nigeria. J. Clin. Microbiol., 2011: p. JCM.01891-10.

53. Andersen, Kristian G., et al., Clinical Sequencing Uncovers Origins and Evolution of Lassa Virus. Cell, 2015. 162(4): p. 738-750.

54. Manning, J.T., N. Forrester, and S. Paessler, Lassa Virus Isolates from Mali and the Ivory Coast Represent an Emerging Fifth Lineage. Frontiers in Microbiology, 2015. 6.

55. Pontremoli, C., et al., Evolutionary analysis of Old World arenaviruses reveals a major adaptive contribution of the viral polymerase. Molecular Ecology, 2017(26): p. 51735188.

56. Bowen, M., et al., Genetic diversity among Lassa virus strains. J. Virol., 2000. 74(15): p. 6992-7004.

57. Gunther, S., et al., Imported Lassa fever in Germany: molecular characterization of a new Lassa virus strain. Emerg. Infect. Dis., 2000. 6(5): p. 466-476.

58. Whitmer, S.L., et al., New Lineage of Lassa Virus, Togo, 2016. Emerging Infectious Disease journal, 2018. 24(3): p. 599.

59. Falzarano, D. and H. Feldmann, Vaccines for viral hemorrhagic fevers - progress and shortcomings. Current opinion in virology, 2013. 3(3): p. 343-351.

60. Richmond, J.K. and D.J. Baglole, Lassa fever: epidemiology, clinical features, and social consequences. BMJ, 2003. 237: p. 1271-1275.

61. Fichet-Calvet, E. and D.J. Rogers, Risk Maps of Lassa Fever in West Africa. PLoS Negl. Trop. Dis., 2009. 3(3): p. doi: e38810.1371/journal.pntd.0000388. 
62. Sogoba, N., H. Feldmann, and D. Safronetz, Lassa Fever in West Africa: Evidence for an Expanded Region of Endemicity. Zoonoses and Public Health, 2012. 59: p. 43-47.

63. Redding, D.W., et al., Environmental-mechanistic modelling of the impact of global change on human zoonotic disease emergence: a case study of Lassa fever. Methods in Ecology and Evolution, 2016. 7(6): p. 646-655.

64. WHO. WHO Target Product Profile for Lassa Virus Vaccine. 2017 [cited 201914 January 2019]; Available from: http://www.who.int/blueprint/priority-diseases/keyaction/LassaVirusVaccineTPP.PDF?ua=1.

65. Emmerich, P., S. Gunter, and H. Schmitz, Strain-specific antibody response to Lassa virus in the local population of West AFrica. J Clin Virol, 2008. 42(1): p. 40-44.

66. Raabe, V. and J. Koehler, Laboratory Diagnosis of Lassa Fever. Journal of Clinical Microbiology, 2017.

67. Lukashevich, I.S., S. Paessler, and J.C. de la Torre, Lassa virus diversity and feasibility for universal prophylactic vaccine. F1000Research, 2019. 8: p. F1000 Faculty Rev-134.

68. Gryseels, S., et al., When Viruses Don't Go Viral: The Importance of Host Phylogeographic Structure in the Spatial Spread of Arenaviruses. PLOS Pathogens, 2017. 13(1): p. e1006073.

69. Wulff, H., et al., Isolation of an arenavirus closely related to Lassa virus from Mastomys natalensis in South-East Africa. Bull. World Health Organ., 1977. 55: p. 441-444.

70. Günther, S., et al., Mopeia virus-related arenavirus in Natal multimammate mice, Morogoro, Tanzania. Emerging infectious diseases, 2009. 15(12): p. 2008.

71. Ishii, A., et al., Novel arenavirus, Zambia. Emerg Infect Dis, 2011. 17.

72. Gryseels, S., et al., Gairo virus, a novel arenavirus of the widespread Mastomys natalensis: Genetically divergent, but ecologically similar to Lassa and Morogoro viruses. Virology, 2015. 476: p. 249-256.

73. Kiley, M.P., J.V. Lange, and K.M. Johnson, Protection of rhesus monkeys from Lassa virus by immunisation with closely related Arenavirus. Lancet, 1979. 2: p. 738.

74. Walker, D.H., et al., Experimental infection of rhesus monkeys with Lassa virus and a closely related arenavirus, Mozambique virus. J. Infect. Dis., 1982. 146(3): p. 360-363.

75. Carnec, X., et al., A Vaccine Platform against Arenaviruses Based on a Recombinant Hyperattenuated Mopeia Virus Expressing Heterologous Glycoproteins. Journal of Virology, 2018. 92(12).

76. Mccormick, J.B., et al., A Prospective Study of the Epidemiology and Ecology of Lassa Fever. J Infect Dis, 1987. 155.

77. Ter Meulen, J., Lukashevich I, Sidibe K, Inapogui A, Marx M, Dorlemann A, Yansane ML, Koulemou K, Chang-Claude J, Schmitz H., Hunting of peridomestic rodents and consumption of their meat as possible risk factors for rodent-to-human transmission of Lassa virus in the Republic of Guinea. Am J Trop Med Hyg, 1996. 55: p. 661-666.

78. Lukashevich, I.S., Chapter 1: "Pathogenesis of the Old World Arenaviruses in Humans". In: S.K. Singh (Ed) "Human Emerging and Re-emerging Infections: Viral \& Parasitic Infections", John Willey \& Sons, Inc, 2016. Volume 1: p. 1-27.

79. Lalis, A. and T. Wirth, Mice and Men: an Evolutionary History of Lassa Fever. 2018. 189212.

80. McCormick, J., Epidemiology and control of Lassa fever. Curr Top Microbiol Immunol, 1987. 134: p. 69 - 78.

81. McCormick, J.B. and S.P. Fisher-Hoch, Lassa fever. Curr Top Microbiol Immunol, 2002. 262: p. 75 - 109. 
82. Demby, A.H., A. Inapogui, K. Kargbo, J. Koninga, K. Kourouma, J. Kanu, M. Coulibaly, K. D. Wagoner, T. G. Ksiazek, C. J. Peters, P. E. Rollin, D. G. Bausch., Lassa fever in Guinea: II. Distribution and prevalence of Lassa virus infection in small mammals. Vector Borne Zoonotic Dis., 2001. 1(4): p. 283-297.

83. Fichet-Calvet, E., et al., Spatial and temporal evolution of Lassa virus in the natural host population in Upper Guinea. Scientific Reports, 2016. 6: p. 21977.

84. Gibb, R., et al., Understanding the cryptic nature of Lassa fever in West Africa. Pathogens and Global Health, 2017: p. 1-13.

85. Ogunro, B.N., Babasola Oluseyi Olugasa, Adeyemi Kayode, Olayinka Olabisi Ishola, Oluseyi Noah Kolawole, Eugene Amiewanlen Odigie, Christian Happi, Detection of Antibody and Antigen for Lassa Virus Nucleoprotein in Monkeys from Southern Nigeria. J Epidemiol and Global Health, 2019. 9(2): p. 125-127.

86. Compans, R.W., Virus entry and release in polarized epithelial cells. Curr Top Microbiol Immunol., 1995. 292: p. 209-219.

87. Warren, T.K., et al., Advanced antisense therapies for postexposure protection against lethal filovirus infections. Nat Med Nature Publishing Group, 2010. 16.

88. Schlie, K., et al., Viral Protein Determinants of Lassa Virus Entry and Release from Polarized Epithelial Cells. J. Virol., 2010. 84(7): p. 3178-3188.

89. Dylla, D.E., et al., Basolateral Entry and Release of New and Old World Arenaviruses from Human Airway Epithelia. J. Virol., 2008. 82: p. 6034-6038.

90. Okokhere, P., et al., Clinical and laboratory predictors of Lassa fever outcome in a dedicated treatment facility in Nigeria: a retrospective, observational cohort study. The Lancet Infectious Diseases, 2018.

91. Benedikz, E.K., et al., Bacterial flagellin promotes viral entry via an NF-kB and Toll Like Receptor 5 dependent pathway. Scientific reports, 2019. 9(1): p. 7903-7903.

92. Smith, S.I., Seriki, A. \& Ajayi, A. , Typhoidal and non-typhoidal Salmonella infections in Africa. Eur J Clin Microbiol Infect Dis 2016. 35: 1913.

93. Delorme-Axford, E. and C.B. Coyne, The Actin Cytoskeleton as a Barrier to Virus Infection of Polarized Epithelial Cells. Viruses, 2011. 3(12): p. 2462-2477.

94. Lukashevich, I., et al., Hemorrhagic fever occurs after intravenous, but not after intragastric, inoculation of rhesus macaques with lymphocytic choriomeningitis virus. J Med Virol, 2002. 67: p. 171 - 186.

95. Lukashevich, I.S., et al., LCMV-mediated hepatitis in rhesus macaques: WE but not ARM strain activates hepatocytes and induces liver regeneration. Archives of Virology, 2004. 149(12): p. 2319-2336.

96. Rodas, J., et al., Mucosal arenavirus infection of primates can protect them from lethal hemorrhagic fever. J Med Virol, 2004. 72: p. 424 - 435.

97. Cosset, F.-L., et al., Characterization of Lassa Virus Cell Entry and Neutralization with Lassa Virus Pseudoparticles. Journal of Virology, 2009. 83(7): p. 3228-3237.

98. Djavani, M., et al., Mucosal Immunization With Salmonella typhimurium Expressing Lassa Virus Nucleocapsid Protein Cross-protects Mice From Lethal Challenge With Lymphocytic Choriomeningitis Virus. Journal of human virology, 2001. 4(2): p. 103-108.

99. Witkowski, P.T., et al., Gastrointestinal Tract As Entry Route for Hantavirus Infection. Frontiers in Microbiology, 2017. 8(1721).

100. Lo lacono, G., et al., Using Modelling to Disentangle the Relative Contributions of Zoonotic and Anthroponotic Transmission: The Case of Lassa Fever. PLoS NegI Trop Dis, 2015. 9(1): p. e3398. 
101. Lo lacono, G., et al., A Unified Framework for the Infection Dynamics of Zoonotic Spillover and Spread. PLOS Neglected Tropical Diseases, 2016. 10(9): p. e0004957.

102. Cao, W., et al., Identification of alpha-dystroglycan as a receptor for lymphocytic choriomeningitis virus and Lassa fever virus. Science, 1998. 282: p. 2079-2081.

103. Imperiali, M., et al., O Mannosylation of \{alpha\}-Dystroglycan Is Essential for Lymphocytic Choriomeningitis Virus Receptor Function. J. Virol., 2005. 79(22): p. 1429714308.

104. Kunz, S., et al., Posttranslational Modification of \{alpha\}-Dystroglycan, the Cellular Receptor for Arenaviruses, by the Glycosyltransferase LARGE Is Critical for Virus Binding. J. Virol., 2005. 79(22): p. 14282-14296.

105. Kunz, S., et al., Characterization of the Interaction of Lassa Fever Virus with Its Cellular Receptor \{alpha\}-Dystroglycan. J. Virol., 2005. 79(10): p. 5979-5987.

106. Rojek, J.M., et al., Old World Arenavirus Infection Interferes with the Expression of Functional \{alpha\}-Dystroglycan in the Host Cell. Mol. Biol. Cell, 2007. 18: p. 4493-4507.

107. Oldstone, M.B.A. and K.P. Campbell, Decoding arenavirus pathogenesis: Essential roles for alpha-dystroglycan-virus interactions and the immune response. Virology, 2011. 411(2): p. 170-179.

108. Oppliger, J., et al., Lassa Virus Cell Entry via Dystroglycan Involves an Unusual Pathway of Macropinocytosis. Journal of Virology, 2016. 90(14): p. 6412-6429.

109. Herrador, A., et al., Dynamic Dystroglycan Complexes Mediate Cell Entry of Lassa Virus. mBio, 2019. 10(2): p. e02869-18.

110. Shimojima, M., et al., Identification of cell surface molecules involved in dystroglycanindependent Lassa virus cell entry. J Virol., 2012. 86: p. 2067-2078.

111. Goncalves, A.-R., et al., Role of DC-SIGN in Lassa virus entry into human dendritic cells. Journal of Virology, 2013. 88(24): p. 11504-11515.

112. Bhattacharyya, S., et al., Enveloped Viruses Disable Innate Immune Responses in Dendritic Cells by Direct Activation of TAM Receptors. Cell Host \& Microbe, 2013. 14(2): p. 136-147.

113. Moller-Tank, S. and W. Maury, Phosphatidylserine receptors: Enhancers of enveloped virus entry and infection. Virology, 2014. 468-470: p. 565-580.

114. Hayes, M.W., et al., Pathogenic Old World Arenaviruses Inhibit TLR2/Mal-Dependent Proinflammatory Cytokines In Vitro. Journal of Virology, 2012. 86(13): p. 7216-7226.

115. Fennewald, S.M., et al., Alterations in NF-\{kappa\}B and RBP-J\{kappa\} by Arenavirus Infection of Macrophages In Vitro and In Vivo. J. Virol., 2002. 76(3): p. 1154-1162.

116. Iwasaki, M., N. Ngo, and J.C. de la Torre, Sodium Hydrogen Exchangers Contribute to Arenavirus Cell Entry. Journal of Virology, 2014. 88: p. 643-654.

117. Jae, L.T., et al., Lassa virus entry requires a trigger-induced receptor switch. Science, 2014. 344(6191): p. 1506-1510.

118. Israeli, H., et al., Mapping of the Lassa virus LAMP1 binding site reveals unique determinants not shared by other old world arenaviruses. PLOS Pathogens, 2017. 13(4): p. e1006337.

119. Jae, L.T. and T.R. Brummelkamp, Emerging intracellular receptors for hemorrhagic fever viruses. Trends in Microbiology, 2015. 23(7): p. 392-400.

120. Sommerstein, R., Flatz, L., Remy, M.M., Malinge, P., Magistrelli, G, Fischer, N., et al., Arenavirus Glycan Shield Promotes Neutralizing Antibody Evasion and Protracted Infection. PLoS Pathog, 2015. 11(11): p. e1005276.

121. Jahrling, P.B., et al., Endemic Lassa fever in Liberia. III. Characterization of Lassa virus isolates. Trans R Soc Trop Med Hyg., 1985. 79(3): p. 374-379. 
122. Peters, C.J., et al., Experimental studies of arenaviral hemorrhagic fevers. Curr. Top. Microbiol. Immunol., 1987. 134: p. 5-68.

123. Lukashevich, I.S., The search for animal models for Lassa fever vaccine development. Expert Rev Vaccines, 2013. 12(1): p. 71-86.

124. Jahrling, P.B., et al., Pathogenesis of Lassa virus infection in guinea pigs. Infect. Immun., 1982. 37(2): p. 771-778.

125. Carrion Jr, R., et al., A ML29 reassortant virus protects guinea pigs against a distantly related Nigerian strain of Lassa virus and can provide sterilizing immunity. Vaccine, 2007. 25(20): p. 4093-4102.

126. Carrion, R., et al., Lassa Virus Infection in Experimentally Infected Marmosets: Liver Pathology and Immunophenotypic Alterations in Target Tissues. Journal of Virology, 2007. 81(12): p. 6482-6490.

127. Clegg, J.C. and G. Lloyd, Vaccinia recombinant expressing Lassa-virus internal nucleocapsid protein protects guinea pigs against Lassa fever. Lancet., 1987. 2(8552): p. 186-188.

128. Safronetz, D., et al., A Recombinant Vesicular Stomatitis Virus-Based Lassa Fever Vaccine Protects Guinea Pigs and Macaques against Challenge with Geographically and Genetically Distinct Lassa Viruses. PLoS Neglected Tropical Diseases, 2015. 9(4): p. e0003736.

129. Safronetz, D., et al., A Recently Isolated Lassa Virus From Mali Demonstrates Atypical Clinical Disease Manifestations and Decreased Virulence in Cynomolgus Macaques. Journal of Infectious Diseases, 2013. 207(8): p. 1316-1327.

130. Keane, E., Gilles H.M., Lassa fever in Panguma Hospital, Sierra Leone, 1973-6. British Medical Journal, 1977. 1: p. 1399-140.

131. McCormick, J.B., et al., A case-control study of the clinical diagnosis and course of Lassa fever. J Infect Dis, 1987. 155.

132. Frame, J.D., Clinical Features of Lassa Fever in Liberia. Rev Infect Dis, 1989. 11: p. S783S789.

133. Frame, J.D., et al., Lassa fever, a new virus disease of man from West Africa. I. Clinical description and pathological findings. The American journal of tropical medicine and hygiene, 1970. 19(4): p. 670-676.

134. McCormick, J.B., et al., Lassa fever. Effective therapy with ribavirin. N. Engl. J. Med., 1986. 314(1): p. 20-26.

135. Bausch, D.G., Demby AH, Coulibaly M, Kanu J, Goba A, Bah A, Condé N, Wurtzel HL et al. , Lassa Fever in Guinea: I. Epidemiology of Human Disease and Clinical Observations Vector-Borne and Zoonotic Diseases., 2001. 1: p. 269-281.

136. Monson, M.H., et al., Pediatric Lassa Fever: A Review of 33 Liberian Cases. The American Journal of Tropical Medicine and Hygiene, 1987. 36(2): p. 408-415.

137. Aaron, K., J.C. Mary, and E.R. Pierre, Lassa Fever in Travelers from West Africa, 19692016. Emerging Infectious Disease journal, 2019. 25(2): p. 236.

138. McCormick, J.B., Clinical, epidemiologic, and therapeutic aspects of Lassa fever. Med Microbiol Immunol, 1986. 175: p. 153 - 5.

139. Mahanty, S., et al., Cutting Edge: Impairment of Dendritic Cells and Adaptive Immunity by Ebola and Lassa Viruses. J. Immunol., 2003. 170(6): p. 2797-2801.

140. McCormick, J., et al., Lassa virus hepatitis: a study of fatal Lassa fever in humans. Am J Trop Med Hyg, 1986. 35(2): p. 401-407. 
141. Baize, S., et al., Early and Strong Immune Responses Are Associated with Control of Viral Replication and Recovery in Lassa Virus-Infected Cynomolgus Monkeys. J. Virol., 2009. 83(11): p. 5890-5903.

142. Lukashevich, I., et al., Arenavirus-mediated liver pathology: acute lymphocytic choriomeningitis virus infection of rhesus macaques is characterized by high-level interleukin-6 expression and hepatocyte proliferation. J Virol, 2003. 77: p. 1727 - 1737.

143. Branco, L.M., et al., Emerging trends in Lassa fever: redefining the role of immunoglobulin $M$ and inflammation in diagnosing acute infection. Virology Journal, 2011. 8(1): p. 478.

144. Winn, W.J., et al., Lassa virus hepatitis. Observations on a fatal case from the 1972 Sierra Leone epidemic. Arch Pathol., 1975. 99(11): p. 599-604.

145. Beier, J.I., Jokinen,J.D., Gretchen E. Holz, G.E., Whang, P.S., Amah M. Martin, A.M., Warner, N.L., Arteel, G.E., Lukashevich, I.S., Novel Mechanism of Arenavirus-Induced Liver Pathology. PLoS One, 2015. 10(3): p. e0122839.

146. Asogun, D.A., et al., Molecular Diagnostics for Lassa Fever at Irrua Specialist Teaching Hospital, Nigeria: Lessons Learnt from Two Years of Laboratory Operation. PLoS Negl Trop Dis, 2012. 6(9): p. e1839.

147. Buba, M.I., et al., Mortality among confirmed Lassa fever cases during the 2015-2016 outbreak in Nigeria. American Journal of Public Health, 2018. 108(2): p. 262-264.

148. Bausch, D.G., The Year That Ebola Virus Took Over West Africa: Missed Opportunities for Prevention. Amer J Trop Med Hyg, 2015. 92(2): p. 219-220.

149. Hartnett, J.N., Boisen, M.L., Oottamasathien, D., Abigail, B.J., Millett, M.M., Nelson, D.S., Muncy, I.J., Goba, A., Momoh, M., Fullah, M., Mire, C.E., Geisbert, J.B., Geisbert, T.W., Holton, D.L., Rouelle, J.A., Kannadka, C.B., Reyna, A.A., Moses, L.M., Khan, S.H., Gevao, S.M., Grant, D.S., Robinson, J.E., Happi, C., Pitts, K.R., Garry, R.F., Branco, L.M. et al., Current and emerging strategies for the diagnosis, prevention and treatment of Lassa fever. Future Virol. , 2015. 10(5): p. 559-584.

150. Ilori, E.A., et al., Increase in Lassa Fever Cases in Nigeria, January-March 2018. Emerging infectious diseases, 2019. 25(5): p. 1026-1027.

151. Zapata, J.C., Carrion, R.Jr, Patterson, J.L., Crasta, O., Zhang, Y., Mani, S., Jett, M., Poonia, B., Djavani, M., White, D.M., Lukashevich, I.S., Salvato, M.S. , Transcriptome Analysis of Human Peripheral Blood Mononuclear Cells Exposed to Lassa Virus and to the Attenuated Mopeia/Lassa Reassortant 29 (ML29), a Vaccine Candidate. PLoS Negl Trop Dis, 2013. 7(9): p. e2406.

152. Siddle, K.J., et al., Genomic Analysis of Lassa Virus during an Increase in Cases in Nigeria in 2018. New England Journal of Medicine, 2018. 379(18): p. 1745-1753.

153. Bausch, D.G., et al., Diagnosis and Clinical Virology of Lassa Fever as Evaluated by Enzyme-Linked Immunosorbent Assay, Indirect Fluorescent-Antibody Test, and Virus Isolation. J. Clin. Microbiol., 2000. 38(7): p. 2670-2677.

154. Johnson, K., et al., Clinical virology of Lassa fever in hospitalized patients. J Infect Dis, 1987. 155: p. 456 - 464.

155. Lukashevich, I.S., Clegg, J.C., Sidibe, K., Lassa virus activity in Guinea: distribution of human antiviral antibody defined using enzyme-linked immunosorbent assay with recombinant antigen. J Med Virol, 1993. 40(3)(210-217).

156. Fisher-Hoch, S.P. and J.B. McCormick, Lassa fever vaccine: A review. Expert Rev Vaccines, 2004. 3: p. 103 - 11.

157. Robinson, J.E., et al., Most neutralizing human monoclonal antibodies target novel epitopes requiring both Lassa virus glycoprotein subunits. Nat Commun, 2016. 7. 
158. Cross, R.W., et al., Treatment of Lassa virus infection in outbred guinea pigs with first-inclass human monoclonal antibodies. Antiviral Research, 2016. 133: p. 218-222.

159. Prescott, J.B., et al., Immunobiology of Ebola and Lassa virus infections. Nat Rev Immunol, 2017. 17(3): p. 195-207.

160. Lukashevich, I.S., Advanced Vaccine Candidates for Lassa Fever. Viruses, 2012. 4(11): p. 2514-2557.

161. Lukashevich, I.S. and P. Pushko, Vaccine platforms to control Lassa fever. Expert Review of Vaccines, 2016. 15(9): p. 1-16.

162. Hensley, L.E., et al., Pathogenesis of lassa fever in cynomolgus macaques. Virology J. , 2011. 8: p. 205.

163. Meulen, J., et al., Old and New World arenaviruses share a highly conserved epitope in the fusion domain of the glycoprotein 2, which is recognized by Lassa virus-specific human CD4+ T-cell clones. Virology, 2004. 321(1): p. 134-143.

164. ter Meulen, J., et al., Characterization of human CD4+ T-cell clones recognizing conserved and variable epitopes of the Lassa virus nucleoprotein. J. Virol., 2000. 74(5): p. 2186-2192.

165. Goicochea, M.A., et al., Evaluation of Lassa virus vaccine immunogenicity in a CBA/JML29 mouse model. Vaccine, 2012. 30(8): p. 1445-1452.

166. Feldmann, F., Lassa rVSV Vaccines. Hemorrhagic Fever Viruses, 2016. Keystone symposia: p. Santa Fe, December 4-8, 2016.

167. Geisbert, T., et al., Development of a new vaccine for the prevention of Lassa fever. PLoS Med, 2005. 2: p. e183.

168. Zapata, J., et al., An attenuated Lassa vaccine in SIV-infected rhesus macaques does not persist or cause arenavirus disease but does elicit Lassa virus-specific immunity. Virology Journal, 2013. 10(1): p. 52.

169. McElroy, A.K., et al., A Case of Human Lassa Virus Infection With Robust Acute T-Cell Activation and Long-Term Virus-Specific T-Cell Responses. The Journal of Infectious Diseases, 2017. 215(12): p. 1862-1872.

170. La Posta, V.J., et al., Cross-protection against lymphocytic choriomeningitis virus mediated by a CD4+ T-cell clone specific for an envelope glycoprotein epitope of Lassa virus. J Virol., 1993. 67(6): p. 3497-3506.

171. Schildknecht, A., et al., Absence of CTL Responses to Early Viral Antigens Facilitates Viral Persistence. J Immunol, 2008. 180(5): p. 3113-3121.

172. Darbre, S., et al., The Nucleoprotein Is Required for Lymphocytic Choriomeningitis VirusBased Vaccine Vector Immunogenicity. Journal of Virology, 2015. 89(22): p. 1173411738.

173. Dennehy, P., Rotavirus Vaccines: an Overview. Clin Microbiol Rev, 2008. 21: p. 198-208.

174. Auperin, D.D., et al., Construction of a recombinant vaccinia virus expressing the Lassa virus glycoprotein gene and protection of guinea pigs from a lethal Lassa virus infection. Virus Res., 1988. 9(2-3): p. 233-248.

175. Pushko, P., et al., Individual and bivalent vaccines based on alphavirus replicons protect guinea pigs against infection with Lassa and Ebola viruses. J. Virol., 2001. 75(23): p. 11677-11685.

176. Pushko, P., et al., Replicon-helper systems from attenuated Venezuelan equine encephalitis virus: expression of heterologous genes in vitro and immunization against heterologous pathogens in vivo. Virology, 1997. 2(2): p. 389-401. 
177. Garbutt, M., et al., Properties of Replication-Competent Vesicular Stomatitis Virus Vectors Expressing Glycoproteins of Filoviruses and Arenaviruses. Journal of Virology, 2004. 78(10): p. 5458-5465.

178. Bredenbeek, P.J., et al., A recombinant Yellow Fever 17D vaccine expressing Lassa virus glycoproteins. Virology, 2006. 345(2): p. 299-304.

179. Jiang, X., et al., Yellow fever 17D-vectored vaccines expressing Lassa virus GP1 and GP2 glycoproteins provide protection against fatal disease in guinea pigs. Vaccine, 2011. 29(6): p. 1248-1257.

180. Kerber, R., et al., Research efforts to control highly pathogenic arenaviruses: A summary of the progress and gaps. Journal of Clinical Virology, 2015. 64: p. 120-127.

181. Martínez-Sobrido, L., B.Y.H. Cheng, and J.C. Carlos de la Torre, Reverse Genetics Approaches to Control Arenavirus. Methods in Molecular Biology, 2016. 1403: p. 313351.

182. Martinez-Sobrido, L. and J.C. de la Torre, Novel strategies for development of hemorrhagic fever arenavirus live-attenuated vaccines. Expert Review of Vaccines, 2016. 15(9): p. 1113-1121.

183. Morrison, H., Bauer SP, Lange JV, Esposito JJ, McCormick JB, Auperin DD., Protection of guinea pigs from Lassa fever by vaccinia virus recombinants expressing the nucleoprotein or the envelope glycoproteins of Lassa virus. Virology, 1989. 171: p. 179188.

184. Abreu-Mota, T., et al., Non-neutralizing antibodies elicited by recombinant Lassa-Rabies vaccine are critical for protection against Lassa fever. Nature Communications, 2018. 9(1): p. 4223.

185. Cashman, K., et al., Enhanced Efficacy of a Codon-Optimized DNA Vaccine Encoding the Glycoprotein Precursor Gene of Lassa Virus in a Guinea Pig Disease Model When Delivered by Dermal Electroporation. Vaccines, 2013. 1(3): p. 262.

186. Kainulainen, M.H., et al., Use of a Scalable Replicon-Particle Vaccine to Protect Against Lethal Lassa Virus Infection in the Guinea Pig Model. The Journal of Infectious Diseases, 2018: p. jiy123-jiy123.

187. Kainulainen, M.H., et al., Protection from lethal Lassa disease can be achieved both before and after virus exposure by administration of single-cycle replicating Lassa virus replicon particles. The Journal of Infectious Diseases, 2019.

188. McCormick, J., et al., Inactivated Lassa virus elicits a non protective immune response in rhesus monkeys. J. Med. Virol., 1992. 31(1): p. 1-7.

189. Cashman, K.A., et al., A DNA vaccine delivered by dermal electroporation fully protects cynomolgus macaques against Lassa fever. Human Vaccines \& Immunotherapeutics, 2017. 13(12): p. 2902-2911.

190. Jiang, J., et al., Immunogenicity of a protective intradermal DNA vaccine against lassa virus in cynomolgus macaques. Human Vaccines \& Immunotherapeutics, 2019: p. 1-9.

191. Fisher-Hoch, S., et al., Protection of rhesus monkeys from fatal Lassa fever by vaccination with a recombinant vaccinia virus containing the Lassa virus glycoprotein gene. Proc Natl Acad Sci USA, 1989. 86: p. 317 - 321.

192. Pinschewer, D.D., M. Perez, and J.C. de la Torre, Role of the virus nucleoprotein in the regulation of Lymphocytic Choriomeningitis Virus transcription and RNA replication. J. Virol., 2003. 77(6): p. 3882-3887.

193. Lee, K.J., et al., Identification of the Lymphocytic Choriomeningitis Virus (LCMV) proteins required to rescue LCMV RNA analogs into LCMV-like particles. J. Virol., 2002. 76(12): p. 6393-6397. 
194. Martínez-Sobrido, L., S. Paessler, and J.C. de la Torre, Lassa Virus Reverse Genetics, in Reverse Genetics of RNA Viruses: Methods and Protocols, D.R. Perez, Editor. 2017, Springer New York: New York, NY. p. 185-204.

195. Walker, D.H., et al., Comparative pathology of Lassa virus infection in monkeys, guineapigs, and Mastomys natalensis. Bull World Health Organ, 1975. 52.

196. Carrion, R., et al., Vaccine Platforms to Control Arenaviral Hemorrhagic Fevers. Journal of vaccines \& vaccination, 2012. 3(7): p. 1000160.

197. Ambrosio, A., et al., Argentine hemorrhagic fever vaccines. Human Vaccines, 2011. 7(6): p. 694-700.

198. Yun, N.E., et al., Mice lacking functional STAT1 are highly susceptible to lethal infection with Lassa virus. Journal of virology, 2013. 87(19): p. 10908-10911.

199. Cohen, J., Unfilled Vials. Science, 2016. 351(6268): p. 16-19.

200. Bausch, D.G., S.S. Sesay, and B. Oshin, On the Front Lines of Lassa Fever. Emerg Infect Dis, 2004. 10(10): p. 1889-1890.

201. Plotkin, S.A., Vaccines for epidemic infections and the role of CEPI. Human Vaccines \& Immunotherapeutics, 2017: p. 1-8.

202. Plotkin, S.A., A.A.F. Mahmoud, and J. Farrar, Establishing a Global Vaccine-Development Fund. New England Journal of Medicine, 2015. 373(4): p. 297-300.

203. Røttingen, J.-A., et al., New Vaccines against Epidemic Infectious Diseases. New England Journal of Medicine, 2017. 376(7): p. 610-613.

204. Johnson, D.M., J.D. Jokinen, and I.S. Lukashevich, Attenuated Replication of Lassa Virus Vaccine Candidate ML29 in STAT-1(-/-) Mice. Pathogens (Basel, Switzerland), 2019. 8(1): p. 9.

205. Roberts, L., Nigeria hit by unprecedented Lassa fever outbreak. 2018, American Association for the Advancement of Science.

206. WHO. Lassa Fever. 201814 January 2019]; Available from: http://www.who.int/csr/don/20-april-2018-lassa-fever-nigeria/en/.

207. WHO. Annual Review of Diseases Prioritized under the Research and Development Blueprint. 201714 January 2019]; Available from: http://www.who.int/blueprint/what/research-development/2017-Prioritization-LongReport.pdf?ua=1.

208. Radoshitzky, S.R., et al., Hemorrhagic fever-causing mammarenaviruses. Medical Aspects of Biological Warfare; Bozue, J., Cote, CK, Glass, PJ, Eds, 2018: p. 517-545.

209. Riviere, Y., et al., Genetic mapping of lymphocytic choriomeningitis virus pathogenicity: virulence in guinea pigs is associated with the L RNA segment. Journal of virology, 1985. 55(3): p. 704-709.

210. Zhang, L., et al., Reassortant analysis of guinea pig virulence of pichinde virus variants. Virology, 2001. 290(1): p. 30-38.

211. Lukashevich, l., et al., The isolation and characteristics of reassortants between the Lassa and Mopeia arenaviruses. Voprosy virusologii, 1991. 36(2): p. 146-150.

212. Moshkoff, D.A., M.S. Salvato, and I.S. Lukashevich, Molecular characterization of a reassortant virus derived from Lassa and Mopeia viruses. Virus genes, 2007. 34(2): p. 169-176.

213. Iwasaki, M., et al. Use of recombinant ML29 platform to generate polyvalent liveattenuated vaccines against lassa fever and other infectious diseases. in Proceedings of the 66th Annual Meeting of Japanese Society for Virology, Kyoto, Japan. 2018.

214. Bell, T., et al., Temporal progression of lesions in guinea pigs infected with Lassa virus. Veterinary pathology, 2017. 54(3): p. 549-562. 
215. Golden, J.W., et al., Animal models for the study of rodent-borne hemorrhagic fever viruses: arenaviruses and hantaviruses. BioMed research international, 2015. 2015.

216. Yun, N.E., et al., Animal Model of Sensorineural Hearing Loss Associated with Lassa Virus Infection. Journal of Virology, 2016. 90(6): p. 2920.

217. López, C.B., Defective Viral Genomes: Critical Danger Signals of Viral Infections. Journal of Virology, 2014. 88(16): p. 8720-8723.

218. Rezelj, V.V., L.I. Levi, and M. Vignuzzi, The defective component of viral populations. Current Opinion in Virology, 2018. 33: p. 74-80.

219. Baltes, A., et al., Inhibition of infection spread by co-transmitted defective interfering particles. PLOS ONE, 2017. 12(9): p. e0184029.

220. McLaren, L.C. and J.J. Holland, Defective interfering particles from poliovirus vaccine and vaccine reference strains. Virology, 1974. 60(2): p. 579-583.

221. Frensing, T., Defective interfering viruses and their impact on vaccines and viral vectors. Biotechnol J, 2015. 10(5): p. 681-9.

222. Gould, P.S., A.J. Easton, and N.J. Dimmock, Live Attenuated Influenza Vaccine contains Substantial and Unexpected Amounts of Defective Viral Genomic RNA. Viruses, 2017. 9(10).

223. Calain, P. and L. Roux, Generation of measles virus defective interfering particles and their presence in a preparation of attenuated live-virus vaccine. J Virol, 1988. 62(8): p. 2859-66.

224. Whistler, T., W.J. Bellini, and P.A. Rota, Generation of defective interfering particles by two vaccine strains of measles virus. Virology, 1996. 220(2): p. 480-4.

225. Ho, T.H., et al., PACT- and RIG-I-Dependent Activation of Type I Interferon Production by a Defective Interfering RNA Derived from Measles Virus Vaccine. J Virol, 2016. 90(3): p. 1557-68.

226. Dutko, F.J. and C.J. Pfau, Arenavirus Defective Interfering Particles Mask the Cell-Killing Potential of Standard Virus. Journal of General Virology, 1978. 38(2): p. 195-208.

227. Popescu, M., H. Schaefer, and F. Lehmann-Grube, Homologous interference of lymphocytic choriomeningitis virus: detection and measurement of interference focusforming units. Journal of virology, 1976. 20(1): p. 1-8.

228. Welsh, R.M., et al., Properties of Defective Lymphocytic Choriomeningitis Virus. Journal of General Virology, 1972. 17(3): p. 355-359.

229. Welsh, R.M., et al., A comparison of biochemical and biological properties of standard and defective lymphocytic choriomeningitis virus. Bulletin of the World Health Organization, 1975. 52(4-6): p. 403-408.

230. Welsh, R.M., P.W. Lampert, and M.B.A. Oldstone, Prevention of Virus-Induced Cerebellar Disease by Defective-Interfering Lymphocytic Choriomeningitis Virus. The Journal of Infectious Diseases, 1977. 136(3): p. 391-399.

231. Welsh, R. and M. Oldstone Inhibition of immunologic injury of cultured cells infected with lymphocytic choriomeningitis virus: role of defective interfering virus in regulating viral antigenic expression. The Journal of Experimental Medicine, 1977. 145(6): p. 14491468.

232. Popescu, M. and F. Lehmann-Grube, Defective interfering particles in mice infected with lymphocytic choriomengingitis virus. Virology, 1977. 77(1): p. 78-83.

233. Lukashevich, I.S., R.F. Mar'iankova, and F.M. Fidarov, [Acute and chronic Lassa virus infection of Vero cells]. Vopr Virusol, 1981(4): p. 452-6.

234. Lukashevich, I.S., et al., Sedimentation analysis of the RNAs isolated from interfering particles of Lassa and Machupo viruses. Acta Virol, 1985. 29(6): p. 455-60. 
235. Lukashevich, I., R. Maryankova, and N. Lemeshko, Autointerfering activity of Lassa virus. Vopr. Virusol, 1983. 28: p. 96-101.

236. Wang, M., et al., Alphavirus vector-based replicon particles expressing multivalent crossprotective Lassa virus glycoproteins. Vaccine, 2018. 36(5): p. 683-690.

237. Kiley, M.P., et al., Serological and biological evidence that Lassa-complex arenaviruses are widely distributed in Africa. Med Microbiol Immunol, 1986. 175(2-3): p. 161-3.

238. Russier, M., D. Pannetier, and S. Baize, Immune responses and Lassa virus infection. Viruses, 2012. 4(11): p. 2766-85.

239. Hallam, H.J., et al., Baseline mapping of Lassa fever virology, epidemiology and vaccine research and development. npj Vaccines, 2018. 3(1): p. 11.

240. Huang, A.S. and D. Baltimore, Defective interfering particles of animal viruses. Comput. Virol., 1977. 10: p. 73-116.

241. Huang, A.S., Defective interfering viruses. Annu Rev Microbiol, 1973. 27: p. 101-17.

242. Barrett, A.t. and N. Dimmock, Defective interfering viruses and infections of animals, in Current topics in microbiology and immunology. 1986, Springer. p. 55-84.

243. Perrault, J., Origin and replication of defective interfering particles, in Initiation Signals in Viral Gene Expression. 1981, Springer. p. 151-207.

244. Roux, L., A.E. Simon, and J.J. Holland, Effects of defective interfering viruses on virus replication and pathogenesis in vitro and in vivo, in Advances in virus research. 1991, Elsevier. p. 181-211.

245. Lukashevich, I. and E. Vela, Pathogenesis of Lassa virus infection in experimental animals. Molecular pathogenesis of hemorrhagic fever viruses. Kerala, India: Transw Res Net, 2010: p. 101-42.

246. Peralta, L.M., M. Bruns, and F. Lehmann-Grube, Biochemical composition of lymphocytic choriomeningitis virus interfering particles. J Gen Virol, 1981. 55(Pt 2): p. 475-9.

247. Meyer, B.J. and P.J. Southern, A novel type of defective viral genome suggests a unique strategy to establish and maintain persistent lymphocytic choriomeningitis virus infections. J Virol, 1997. 71(9): p. 6757-64.

248. Cornu, T.I., H. Feldmann, and J.C. de la Torre, Cells expressing the RING finger Z protein are resistant to arenavirus infection. J Virol, 2004. 78(6): p. 2979-83.

249. Zaza, A.D., et al., Mammarenaviruses deleted from their $Z$ gene are replicative and produce an infectious progeny in BHK-21 cells. Virology, 2018. 518: p. 34-44.

250. Xing, J., H. Ly, and Y. Liang, The Z proteins of pathogenic but not nonpathogenic arenaviruses inhibit RIG-I-like receptor-dependent interferon production. J Virol, 2015. 89(5): p. 2944-55.

251. Zapata, J.C., et al., Improving the Breadth of the Host's Immune Response to Lassa Virus. Pathogens, 2018. 7(4).

252. Sun, Y., et al., Immunostimulatory Defective Viral Genomes from Respiratory Syncytial Virus Promote a Strong Innate Antiviral Response during Infection in Mice and Humans. PLoS Pathog, 2015. 11(9): p. e1005122.

253. Yoneyama, M., et al., The RNA helicase RIG-I has an essential function in doublestranded RNA-induced innate antiviral responses. Nat Immunol, 2004. 5(7): p. 730-7.

254. Reynard, S., et al., Exonuclease domain of the Lassa virus nucleoprotein is critical to avoid RIG-I signaling and to inhibit the innate immune response. Journal of virology, 2014. 88(23): p. 13923-13927.

255. Mateer, E.J., S. Paessler, and C. Huang, Visualization of Double-Stranded RNA Colocalizing With Pattern Recognition Receptors in Arenavirus Infected Cells. Frontiers in cellular and infection microbiology, 2018. 8: p. 251-251. 
256. Martinez-Sobrido, L., et al., Differential inhibition of type I interferon induction by arenavirus nucleoproteins. J Virol, 2007. 81(22): p. 12696-703.

257. Meyer, B. and H. Ly, Inhibition of Innate Immune Responses Is Key to Pathogenesis by Arenaviruses. Journal of virology, 2016. 90(8): p. 3810-3818.

258. Honda, K., A. Takaoka, and T. Taniguchi, Type I inteferon gene induction by the interferon regulatory factor family of transcription factors. Immunity, 2006. 25(3): p. 349-360.

259. Heylbroeck, C., et al., The IRF-3 Transcription Factor Mediates Sendai Virus-Induced Apoptosis. Journal of Virology, 2000. 74(8): p. 3781.

260. Li, Q. and I.M. Verma, NF-KB regulation in the immune system. Nature Reviews Immunology, 2002. 2(10): p. 725-734.

261. Martinez-Sobrido, L., et al., Inhibition of the type I interferon response by the nucleoprotein of the prototypic arenavirus lymphocytic choriomeningitis virus. J Virol, 2006. 80(18): p. 9192-9.

262. Pythoud, C., et al., Arenavirus nucleoprotein targets interferon regulatory factoractivating kinase IKKepsilon. J Virol, 2012. 86(15): p. 7728-38.

263. Vignuzzi, M. and C.B. López, Defective viral genomes are key drivers of the virus-host interaction. Nature Microbiology, 2019. 4(7): p. 1075-1087.

264. Xu, J., et al., Replication defective viral genomes exploit a cellular pro-survival mechanism to establish paramyxovirus persistence. Nature communications, 2017. 8(1): p. 1-13.

265. Fuller, F.J. and P.I. Marcus, Interferon induction by viruses. IV. Sindbis virus: early passage defective-interfering particles induce interferon. Journal of General Virology, 1980. 48(1): p. 63-73.

266. Tapia, K., et al., Defective viral genomes arising in vivo provide critical danger signals for the triggering of lung antiviral immunity. PLoS Pathog, 2013. 9(10): p. e1003703.

267. Yount, J.S., et al., MDA5 participates in the detection of paramyxovirus infection and is essential for the early activation of dendritic cells in response to Sendai Virus defective interfering particles. J Immunol, 2008. 180(7): p. 4910-8.

268. Strahle, L., D. Garcin, and D. Kolakofsky, Sendai virus defective-interfering genomes and the activation of interferon-beta. Virology, 2006. 351(1): p. 101-11.

269. Baum, A., R. Sachidanandam, and A. Garcia-Sastre, Preference of RIG-I for short viral RNA molecules in infected cells revealed by next-generation sequencing. Proc Natl Acad Sci U S A, 2010. 107(37): p. 16303-8.

270. Runge, S., et al., In vivo ligands of MDA5 and RIG-I in measles virus-infected cells. PLoS Pathog, 2014. 10(4): p. e1004081.

271. Yount, J.S., et al., A novel role for viral-defective interfering particles in enhancing dendritic cell maturation. J Immunol, 2006. 177(7): p. 4503-13.

272. Marriott, A.C. and N.J. Dimmock, Defective interfering viruses and their potential as antiviral agents. Reviews in Medical Virology, 2010. 20(1): p. 51-62.

273. Ziegler, C.M., et al., The lymphocytic choriomeningitis virus matrix protein PPXY late domain drives the production of defective interfering particles. PLoS pathogens, 2016. 12(3).

274. Buchmeier, M., Arenaviruses: protein structure and function, in Arenaviruses I. 2002, Springer. p. 159-173.

275. Olayemi, A., et al., Determining Ancestry between Rodent- and Human-Derived Virus Sequences in Endemic Foci: Towards a More Integral Molecular Epidemiology of Lassa Fever within West Africa. Biology (Basel), 2020. 9(2). 
276. Golden, J.W., et al., An attenuated Machupo virus with a disrupted L-segment intergenic region protects guinea pigs against lethal Guanarito virus infection. Scientific reports, 2017. 7(1): p. 4679-4679.

277. Dimmock, N.J. and A.J. Easton, Cloned defective interfering influenza RNA and a possible pan-specific treatment of respiratory virus diseases. Viruses, 2015. 7(7): p. 3768-3788.

278. Mateer, E.J., et al., Lassa fever-induced sensorineural hearing loss: A neglected public health and social burden. PLoS Negl Trop Dis, 2018. 12(2): p. e0006187.

279. Cummins, D., et al., Acute sensorineural deafness in Lassa fever. Jama, 1990. 264(16): p. 2093-6.

280. Ibekwe, T.S., et al., Early-onset sensorineural hearing loss in Lassa fever. European Archives of Oto-Rhino-Laryngology, 2011. 268(2): p. 197-201.

281. Maruyama, J., et al., Lethal Infection of Lassa Virus Isolated from a Human Clinical Sample in Outbred Guinea Pigs without Adaptation. mSphere, 2019. 4(5): p. e00428-19.

282. Safronetz, D., et al., The broad-spectrum antiviral favipiravir protects guinea pigs from lethal Lassa virus infection post-disease onset. Sci Rep, 2015. 5: p. 14775.

283. Emonet, S.F., et al., Generation of recombinant lymphocytic choriomeningitis viruses with trisegmented genomes stably expressing two additional genes of interest. Proceedings of the National Academy of Sciences of the United States of America, 2009. 106(9): p. 3473-3478.

284. Sanchez, A.B. and J.C. de la Torre, Rescue of the prototypic Arenavirus LCMV entirely from plasmid. Virology, 2006. 350(2): p. 370-80.

285. Flatz, L., et al., Recovery of an arenavirus entirely from RNA polymerase I/II-driven CDNA. Proceedings of the National Academy of Sciences of the United States of America, 2006. 103(12): p. 4663.

286. Hitoshi, N., Y. Ken-ichi, and M. Jun-ichi, Efficient selection for high-expression transfectants with a novel eukaryotic vector. Gene, 1991. 108(2): p. 193-199.

287. Au - Schultheis, K., et al., Optimized Interferon-gamma ELISpot Assay to Measure T Cell Responses in the Guinea Pig Model after Vaccination. JoVE, 2019(143): p. e58595.

288. Kerber, R., et al., Cross-Species Analysis of the Replication Complex of Old World Arenaviruses Reveals Two Nucleoprotein Sites Involved in L Protein Function. Journal of Virology, 2011. 85(23): p. 12518.

289. Meraz, M.A., et al., Targeted disruption of the Stat1 gene in mice reveals unexpected physiologic specificity in the JAK-STAT signaling pathway. Cell, 1996. 84(3): p. 431-42.

290. Duvignaud, A., et al., Lassa fever clinical course and setting a standard of care for future randomized trials: A protocol for a cohort study of Lassa-infected patients in Nigeria (LASCOPE). Travel Medicine and Infectious Disease, 2020: p. 101557.

291. Shurtleff, A.C., et al., 6 Animal Models of Viral Hemorrhagic Fevers, in Viral Hemorrhagic Fevers. 2016, CRC Press. p. 100-117.

292. Smadel, J., et al., Lymphocytic Choriomeningitis: Two Human Fatalities Following an Unusual Febrile Illness. Proceedings of the Society for Experimental Biology and Medicine, 1942. 49(4): p. 683-686.

293. Dimmock, N.J. and A.J. Easton, Defective interfering influenza virus RNAs: time to reevaluate their clinical potential as broad-spectrum antivirals? Journal of virology, 2014. 88(10): p. 5217-5227.

294. Smith, C.M., et al., A defective interfering influenza RNA inhibits infectious influenza virus replication in human respiratory tract cells: a potential new human antiviral. Viruses, 2016. 8(8): p. 237. 
295. Aguilar, P.V., et al., Endemic Venezuelan equine encephalitis in the Americas: hidden under the dengue umbrella. Future virology, 2011. 6(6): p. 721-740.

296. Beckham, J.D. and K.L. Tyler, 91 - Encephalitis, in Mandell, Douglas, and Bennett's Principles and Practice of Infectious Diseases (Eighth Edition), J.E. Bennett, R. Dolin, and M.J. Blaser, Editors. 2015, Content Repository Only!: Philadelphia. p. 1144-1163.e3.

297. Taylor, K.G. and S. Paessler, Pathogenesis of Venezuelan equine encephalitis. Veterinary Microbiology, 2013. 167(1): p. 145-150.

298. Zacks, M.A. and S. Paessler, Encephalitic alphaviruses. Veterinary microbiology, 2010. 140(3-4): p. 281-286.

299. Estrada-Franco, J.G., et al., Venezuelan equine encephalitis virus, southern Mexico. Emerging infectious diseases, 2004. 10(12): p. 2113.

300. Samy, A.M., et al., Climate change influences on the global potential distribution of the mosquito Culex quinquefasciatus, vector of West Nile virus and lymphatic filariasis. PloS one, 2016. 11(10): p. e0163863.

301. Hongoh, V., et al., Expanding geographical distribution of the mosquito, Culex pipiens, in Canada under climate change. Applied Geography, 2012. 33: p. 53-62.

302. Brown, H.E., et al., Projection of climate change influences on US West Nile virus vectors. Earth interactions, 2015. 19(18): p. 1-18.

303. Weaver, S.C. and S. Paessler, Chapter 21 - Alphaviral Encephalitides, in Vaccines for Biodefense and Emerging and Neglected Diseases, A.D.T. Barrett and L.R. Stanberry, Editors. 2009, Academic Press: London. p. 339-359.

304. Sharma, A. and B. Knollmann-Ritschel, Current Understanding of the Molecular Basis of Venezuelan Equine Encephalitis Virus Pathogenesis and Vaccine Development. Viruses, 2019. 11(2): p. 164.

305. Bronze, M.S., et al., Viral agents as biological weapons and agents of bioterrorism. Am J Med Sci, 2002. 323(6): p. 316-25.

306. Control, C.f.D. and Prevention, Possession, use, and transfer of select agents and toxins; biennial review. Final rule. Federal register, 2012. 77(194): p. 61083.

307. Lennette, E.H. and H. Koprowski, Human Infection with Venezuelan Equine Encephalomyelitis Virus: a Report on Eight Cases of Infection Acquired in the Laboratory. JAMA, 1943. 123(17): p. 1088-1095.

308. Sulkin, S.E., Laboratory-acquired infections. Bacteriological reviews, 1961. 25(3): p. 203209.

309. Rusnak, J.M., et al., Comparison of Aerosol- and Percutaneous-acquired Venezuelan Equine Encephalitis in Humans and Nonhuman Primates for Suitability in Predicting Clinical Efficacy under the Animal Rule. Comparative medicine, 2018. 68(5): p. 380-395.

310. Sutton, L.S. and C.C. Brooke, Venezuelan equine encephalomyelitis due to vaccination in man. Journal of the American Medical Association, 1954. 155(17): p. 1473-1476.

311. Kinney, R.M., et al., Attenuation of Venezuelan equine encephalitis virus strain TC-83 is encoded by the 5'-noncoding region and the 22 envelope glycoprotein. Journal of virology, 1993. 67(3): p. 1269-1277.

312. Main, C., et al. Safety of an attenuated Venezuelan equine encephalititis virus (VEEV) vaccine in humans; 48th ICAAC. in IDSA Meeting. 2008. Washington, DC, USA: Infectious Disease Society of America.

313. Hyde, J.L., et al., A viral RNA structural element alters host recognition of nonself RNA. Science (New York, N.Y.), 2014. 343(6172): p. 783-787. 
314. Rusnak, J.M., et al., Approach to Strain Selection and the Propagation of Viral Stocks for Venezuelan Equine Encephalitis Virus Vaccine Efficacy Testing under the Animal Rule. Viruses, 2019. 11(9): p. 807.

315. Alevizatos, A.C., R.W. McKinney, and R.D. Feigin, Live, attenuated Venezuelan equine encephalomyelitis virus vaccine. I. Clinical effects in man. Am J Trop Med Hyg, 1967. 16(6): p. 762-8.

316. Martin, S.S., et al., Telemetric analysis to detect febrile responses in mice following vaccination with a live-attenuated virus vaccine. Vaccine, 2009. 27(49): p. 6814-6823.

317. Kenney, J.L., et al., Stability of RNA virus attenuation approaches. Vaccine, 2011. 29(12): p. 2230-2234.

318. Erasmus, J.H. and S.C. Weaver, Biotechnological Applications of an Insect-Specific Alphavirus. DNA Cell Biol, 2017. 36(12): p. 1045-1049.

319. Guerbois, M., et al., IRES-driven expression of the capsid protein of the Venezuelan equine encephalitis virus TC-83 vaccine strain increases its attenuation and safety. PLoS Negl Trop Dis, 2013. 7(5): p. e2197.

320. Rossi, S.L., et al., IRES-Containing VEEV Vaccine Protects Cynomolgus Macaques from IE Venezuelan Equine Encephalitis Virus Aerosol Challenge. PLoS neglected tropical diseases, 2015. 9(5): p. e0003797-e0003797.

321. Rossi, S.L., et al., IRES-based Venezuelan equine encephalitis vaccine candidate elicits protective immunity in mice. Virology, 2013. 437(2): p. 81-8.

322. Tretyakova, I., et al., DNA Vaccine Initiates Replication of Live Attenuated Chikungunya Virus In Vitro and Elicits Protective Immune Response in Mice. The Journal of Infectious Diseases, 2014. 209(12): p. 1882-1890.

323. Tretyakova, I., et al., Venezuelan equine encephalitis vaccine with rearranged genome resists reversion and protects non-human primates from viremia after aerosol challenge. Vaccine, 2020.

324. Cardiff, R.D., C.H. Miller, and R.J. Munn, Manual hematoxylin and eosin staining of mouse tissue sections. Cold Spring Harb Protoc, 2014. 2014(6): p. 655-8.

325. Afgan, E., et al., The Galaxy platform for accessible, reproducible and collaborative biomedical analyses: 2018 update. Nucleic Acids Research, 2018. 46(W1): p. W537W544.

326. Bolger, A.M., M. Lohse, and B. Usadel, Trimmomatic: a flexible trimmer for Illumina sequence data. Bioinformatics, 2014. 30(15): p. 2114-2120.

327. Langmead, B. and S.L. Salzberg, Fast gapped-read alignment with Bowtie 2. Nature Methods, 2012. 9(4): p. 357-359.

328. Kim, D., B. Langmead, and S.L. Salzberg, HISAT: a fast spliced aligner with low memory requirements. Nature methods, 2015. 12(4): p. 357-360.

329. Anders, S., P.T. Pyl, and W. Huber, HTSeq--a Python framework to work with highthroughput sequencing data. Bioinformatics (Oxford, England), 2015. 31(2): p. 166-169.

330. Love, M.I., W. Huber, and S. Anders, Moderated estimation of fold change and dispersion for RNA-seq data with DESeq2. Genome biology, 2014. 15(12): p. 550-550.

331. Young, M.D., et al., Gene ontology analysis for RNA-seq: accounting for selection bias. Genome Biology, 2010. 11(2): p. R14.

332. Geiss, G.K., et al., Direct multiplexed measurement of gene expression with color-coded probe pairs. Nat Biotechnol, 2008. 26(3): p. 317-25.

333. Tsang, H.F., et al., NanoString, a novel digital color-coded barcode technology: current and future applications in molecular diagnostics. Expert Rev Mol Diagn, 2017. 17(1): p. 95-103. 
334. Veldman-Jones, M.H., et al., Evaluating Robustness and Sensitivity of the NanoString Technologies nCounter Platform to Enable Multiplexed Gene Expression Analysis of Clinical Samples. Cancer Res, 2015. 75(13): p. 2587-93.

335. Makarava, N., et al., Region-specific glial homeostatic signature in prion diseases is replaced by a uniform neuroinflammation signature, common for brain regions and prion strains with different cell tropism. Neurobiology of Disease, 2020. 137: p. 104783.

336. Aaby, P. and C.S. Benn, Developing the concept of beneficial non-specific effect of live vaccines with epidemiological studies. Clin Microbiol Infect, 2019. 25(12): p. 1459-1467.

337. Lauring, A.S., J.O. Jones, and R. Andino, Rationalizing the development of live attenuated virus vaccines. Nature Biotechnology, 2010. 28(6): p. 573-579.

338. Steele, K.E., et al., Comparative neurovirulence and tissue tropism of wild-type and attenuated strains of Venezuelan equine encephalitis virus administered by aerosol in C3H/HeN and BALB/C mice. Vet Pathol, 1998. 35(5): p. 386-97.

339. Bowen, G.S., et al., Clinical aspects of human Venezuelan equine encephalitis in Texas. Bull Pan Am Health Organ, 1976. 10(1): p. 46-57.

340. McKinney, R.W., et al., USE OF AN ATTENUATED STRAIN OF VENEZUELAN EQUINE ENCEPHALOMYELITIS VIRUS FOR IMMUNIZATION IN MAN. Am J Trop Med Hyg, 1963. 12: p. 597-603.

341. Forrester, N.L., et al., Evolution and spread of Venezuelan equine encephalitis complex alphavirus in the Americas. PLoS neglected tropical diseases, 2017. 11(8): p. e0005693e0005693.

342. Parker, M.D., et al., Antibody to the E3 glycoprotein protects mice against lethal venezuelan equine encephalitis virus infection. Journal of virology, 2010. 84(24): p. 12683-12690.

343. Kautz, T.F., et al., Low-fidelity Venezuelan equine encephalitis virus polymerase mutants to improve live-attenuated vaccine safety and efficacy. Virus evolution, 2018. 4(1): p. vey004-vey004.

344. Iwasaki, M., et al., General Molecular Strategy for Development of Arenavirus LiveAttenuated Vaccines. J Virol, 2015. 89(23): p. 12166-77.

345. Martinez-Sobrido, L. and J.C. de la Torre, Development of Recombinant ArenavirusBased Vaccines. Methods Mol Biol, 2017. 1581: p. 133-149.

346. Iwasaki, M., et al. Establishment of recombinant ML29 platform for the generation of polyvalent live-attenuated vaccines against Lassa virus and other infectious agents. in The 18th Awaji International Forum on Infection and Immunity. 2019. Awaji, Japan.

347. Jacamo, R., et al., Tacaribe virus $Z$ protein interacts with the $L$ polymerase protein to inhibit viral RNA synthesis. J Virol, 2003. 77(19): p. 10383-93.

348. Shaffer, J.G., et al., A medical records and data capture and management system for Lassa fever in Sierra Leone: Approach, implementation, and challenges. PLoS One, 2019. 14(3): p. e0214284.

349. Lim, K.-I., et al., Model-based design of growth-attenuated viruses. PLoS computational biology, 2006. 2(9): p. e116-e116.

350. Rozen-Gagnon, K., et al., Alphavirus Mutator Variants Present Host-Specific Defects and Attenuation in Mammalian and Insect Models. PLOS Pathogens, 2014. 10(1): p. e1003877. 


\section{CURRICULUM VITA}

Dylan M Johnson

dylanj@hawaii.edu

\section{EDUCATION}

University of Louisville, School of Medicine, Louisville, KY

Ph.D. in Microbiology and Immunology

2016- 2020

Lab of Igor S Lukashevich MD., PhD, DSc

Dissertation: "Development of Rationally Designed Live Attenuated

Vaccines for Lassa Fever and Venezuelan Equine Encephalitis"

University of Louisville, School of Medicine, Louisville, KY

M.S. in Microbiology and Immunology (GPA: 3.96)

2016-2018

Indiana State University, Terre Haute, IN

M.S. in Biology (GPA 4.00)

2012-2014

Lab of Swapan K. Ghosh, PhD

Thesis: "Phytol Derived Immunoadjuvants as Oil-in-Water Micro-Emulsions for use

in Vaccines"

John A. Burns School of Medicine, Honolulu, HI

Post-Baccalaureate Graduate Studies

2008-2009

Department of Tropical Medicine, Medical Microbiology and Pharmacology

University of Hawaii at Manoa, Honolulu, $\mathrm{HI}$

B.S. in Microbiology

2003-2008

\section{PEER REVIEWED PUBLICATIONS}

ORCID 0000-0002-3630-0588

Johnson DM, Sokoloski KJ, Jokinen JD, Pfeffer TL, Chu YK, Adcock RS, Chung D, Tretyakova I, Pushko P, Lukashevich IS. Advanced Safety and Genetic Stability in Mice of a Novel DNA-Launched Venezuelan Equine Encephalitis Virus Vaccine with Rearranged Structural Genes. Vaccines. 2020, 8(1) 114. PMID: 32121666

Johnson DM, Jokinen JD, Wang M, Pfeffer T, Tretyakova I, Carrion R, Griffiths A, Pushko P, Lukashevich IS. Bivalent Junin \& Machupo experimental vaccine based on alphavirus RNA replicon vector. Vaccine. 2020, (38)14,3249-2959. PMID: 32111526

Johnson DM, Jokinen JD, Lukashevich IS. Attenuated Replication of Lassa Virus Vaccine Candidate ML29 in STAT-1 -/- Mice. Pathogens. 2019, 8(1),9. PMID: 30650607

Tretyakoa I, Tibbens A, Jokinen JD, Johnson DM, Lukashevich IS, Pushko P. Novel DNA-launched Venezuelan equine encephalitis virus vaccine with rearranged genome. Vaccine. 2019, (37) 3317-3325. PMID: 31072736

\section{GRANT AND FELLOWSHIP FUNDING}

Genentech Riverport distribution facility community outreach grant to support Louisville Science Pathways

Director of Louisville Science Pathways

Summer 2019

University of Louisville Center for Predictive Medicine Fellowship

0.5 Stipend and tuition waiver for Microbiology and Immunology Ph.D. program

2018-2020

University of Louisville Institutional Program in Biomedical Science Fellowship

2016-2018

Stipend and tuition waiver for Microbiology and Immunology Ph.D. program

University of Louisville Graduate Student Council Travel Funding Award

Summer 2019

Award to present an oral abstract at the annual meeting for the American Society of Virology 


\section{INVITED SYMPOSIA, PRESENTATIONS, AND OTHER PUBLICATIONS}

Johnson DM, Jokinen JD, Pfeffer TL, Chu YK, Chung D, Tretyakova I, Sokoloski KJ, Pushko P, Lukashevich IS. Novel Venezuelan Equine Encephalitis Vaccine V4020 Has Increased Safety and Stability over TC-83. Poster session presented at ASM Biothreats; January 28-30th 2020; Arlington, VA.

Tretyakova I, Johnson DM, Tibbens A, Lukashevich IS, Pushko P. Development of Manufacturing Process for Reassortant Lassa Vaccine ML29. Poster at ASM BioThreats; January 29, 2020; Arlington, VA.

Johnson DM, Sattler RS, Cubitt B, Pfeffer TL, Jokinen JD, Paessler S, de la Torre JC, Lukashevich IS. Lassa virus vaccine candidate ML29 enriched with interfering particles demonstrates excellent safety in STAT1-/- mice, a model to assess sensorineural hearing loss in infected individuals. Poster session presented at the $1^{\text {st }}$ Midwest Virology Symposium; October 12, 2019; Columbus, $\mathrm{OH}$.

Johnson DM, Jokinen JD, Lukashevich IS. Lassa Virus Vaccine Candidate ML29 is More Attenuated and Immunogenic in STAT-1/- Mice than Non-pathogenic Mopeia virus. Oral abstract presented at the American Society of Virology Annual Meeting; July 22, 2019; Minneapolis, MN.

Iwasaki M, Cubitt B, Motooka D, Johnson DM, Lukashevich IS, de la Torre JC. Establishment of recombinant ML29 platform for the generation of polyvalent live-attenuated vaccines against Lassa virus and other infectious agents. Poster at The $18^{\text {th }}$ Awaji International Forum on Infection and Immunity; September 10, 2019; Awaji, Japan.

Johnson DM, Jokinen JD, Pfeffer TL, Chu YK, Chung D, Tretyako I, Sokoloski JK, Pushko P, Lukshevich IS. Novel Venezuelan Equine Encephalitis Vaccine has Increased Safety and Stability over TC-83. Poster session presented at Research! Louisville, September 10, 2019; Louisville, KY.

Tretyakova I, Jokinen J, Tibbens A, Gannon R, Johnson D, Lukashevich I, Pushko P. DNA-launched vaccines with rearranged structural genes for Venezuelan Equine Encephalitis and Chikungunya. Poster at ASM BioThreats Meeting; January 30, 2019; Washington, DC.

Johnson DM Jokinen JD, Tretyakova I, Pushko, P, Lukashevich IS. Novel Venezuelan Equine Encephalitis Vaccine has Increased Safety and Stability over TC-83. Poster session presented at Research! Louisville; October 9, 2018; Louisville, KY.

Johnson DM, Jokinen JD, Lukashevich IS. ML29 Defective Interfering Particles as a Model System to Study Mechanisms of Lassa Virus Persistence. Poster session presented at Research! Louisville; September 12, 2017; Louisville, KY.

Johnson, DM. Ghosh Immunology Symposium at Indiana State University. Talk Title: Characterization of defective interfering particles from ML29, a Lassa fever vaccine candidate. March 31, 2017; Terre Haute, IN.

Ghosh, S, Johnson, D, Steding, C, Fitch, R. Adjuvanticity and chemo-therapeutic potential of novel Phytol-derived immunoadjuvants. Conference: ICI 2016. Location Melbourne, Australia. Date Aug 21-26, 2016. Eur. J. Immunol. 2016. 46, S1, p234. Meeting Abstract number 241.

Johnson DM, Munn S, Grant T, Eberling A, Barret DH, Ortmann L. Centers for Disease Control and Prevention (CDC). Science Ambassador Workshop-RAGE Outbreak: Making grueling public health decisions. Atlanta, GA: US Department of Health and Human Services, CDC; 2015. Available at: https://www.cdc.gov/careerpaths/scienceambassador/educational/careers.html

Johnson DM, Fitch RW, Ghosh SK. Submicron-emulsions of phytanol immunoadjuvants are as effective at significantly reduced concentrations as commercial squalene containing vaccine formulations. Oral abstract presented at the Indiana Academy of Science. 129th Annual Academy Meeting; 2014 March 15; Indianapolis, IN.

Reeves RA, Johnson DM, Fitch RW Ghosh SK. Assessment of Cellular Toxicity of a Potential Immunoadjuvant of Phytanyl Sulfate. Poster at: NCUR 2014. 28th National Conference on Undergraduate Research; 2014 April 3; Lexington, KY.

Johnson DM, Reeves RA, Fitch RW, Ghosh SK. Formulation of Phytanol as a Nanoparticle Emulsion and Sodium Phytanyl Sulfate. Poster session presented at Indiana State University Center for Student Research and Creativity Symposium; 2014 April 7, Terre Haute, IN. 


\section{RESEARCH EXPERIENCE}

The Scripps Research Institute, La Jolla, CA

Training in Arenavirus reverse genetics with Juan Carlos de la Torre, Ph.D.

March 2019

Functional mini-genome systems, rescue from cDNA, and design of recombinant tri-

segmented Arenaviruses.

NIH Regional Biocontainment Facility on the University of Louisville Campus

Center for Predictive Medicine for Biodefense and Emerging Infectious Diseases

Development of small animal models of Arenavirus and Alphavirus infection including work to

assess neurovirulence and immunogenicity of vaccines. Training in select agent regulations,

working at BSL3/ABSL3, and protocol development. Training in the operation and maintenance

of a BD FACS Aria II cell sorter at BSL3.

Indiana State University, Terre Haute, IN

Educational Affiliate

Phytol derived vaccine adjuvants for infectious disease and cancer with immunogenicity

studies in mouse and rabbit models. Lab management including mentoring of graduate

students.

Union Hospital, Terre Haute, IN

Research Assistant to Rizwan Malik, M.D.

Summer 2012

Consulted on establishment of a lab for cryopreservation of embryos for IVF. Reviewed

literature and prepared

a comprehensive manuscript on the emerging role of vitrification in the cryopreservation of

human embryos.

Pharmacology Lab, John A. Burns School of Medicine, Honolulu Hawaii

Graduate Researcher

$2008-2009$

High-throughput compound analysis by enzyme kinetics for antimalarial development.

Immunology Lab, John A. Burns School of Medicine, Honolulu Hawaii

Graduate Researcher

2007-2008

Plasmodium falciparum malaria culture for metabolomics and Luminex based survey of MSP-1

antibodies from patient samples from SE Asia.

\section{TEACHING EXPERIENCE}

University of Louisville, Louisville, KY

Invited Lecturer - Microbiology and Immunology Methods Course (MICR610): Animal Models of Infections

Course for first year Ph.D. students

Terre Haute North Vigo High School, Terre Haute, IN

Science Teacher - Regular Contract

2014-2016

Physics, chemistry, and biology teacher for 9-12 grade students.

Indiana State University, Terre Haute, IN

Teaching Assistant - Graduate/Upper Division Immunology Lab (BIO508L/ BIO408L)

Spring 2013

Collaborated on course structure, syllabus, and student lab manual for material including immunoassays, antibody purification and detection, cell proliferation and activation assays, and techniques for working with mice and rabbits in a research setting. Delivered course content and administered assessments.

Indiana State University, Terre Haute, IN

Invited Lecture- Human Aspects of Biology (BIO112): Epidemiology

Indiana State University, Terre Haute, IN

Teaching Assistant - Upper Division Genetics Lab (BIO380L)

Collaborated on course structure and materials, delivered weekly lectures, assisted students with hands

on experiences, and graded all coursework. Helped develop and implement next generation sequencing sample preparations as new content for the course. Other experiments included: PAGE with Western Blot, bacterial transformations and plasmid isolation. 
Indiana State University, Terre Haute, IN

Delivered weekly lectures, assisted students with hands on experiences, and graded

all coursework.

Introductory course for non-major undergraduates

Student Recreation Services, University of Hawaii at Manoa, Honolulu, HI Instructor of recreational SCUBA

2007-2012

Lead non-credit co-curricular courses leading to certification in open water SCUBA

diving.

\section{RELATED EXPERIENCE}

University of Louisville, Louisville, KY Director of Louisville Science Pathways

2018- 2020

LSP is an outreach initiative that places high school students in research labs as summer interns. The LSP director reports to the Science Policy and Outreach Group and is responsible for recruiting faculty and graduate student mentors, soliciting application from and selecting high school students for the program, coordinating the program, and securing funding for the project.

Center for Disease Control, Atlanta, GA

Science Ambassador

Attended workshops on teaching public health topics. Developed a lesson plan on research ethics in public health for publication by the CDC.

Indiana State University, Terre Haute, IN

Student and Academic Services Tutor

$2012-2013$

Tutored students in biology, chemistry and nursing coursework.

Pearl Harbor Divers, LLC, Honolulu, HI

Owner and Director of Training

Coordinated instructors and curriculum for classes ranging from introductory through instructor level SCUBA.

\section{SERVICE}

University of Louisville Graduate Student Council

University of Louisville Institutional Biosafety Committee Member

University of Louisville School of Medicine Faculty Forum: Elected Graduate Student Representative

Microbiology and Immunology Student Group Elected Officer

\section{AWARDS}

Guy Stevenson Award for Excellence in Graduate Studies,

University of Louisville's highest award for a doctoral student, 2020

ETS Recognition of Excellence: Biology Content Knowledge 2014

ETS Recognition of Excellence: Mathematics Content Knowledge 2014

Outstanding Student Assistant, Indiana State University 2013

Excellence award for outstanding service and professionalism in PADI Scuba Instruction

\section{ACCREDITATIONS}

Full BSL-3/ABSL-3 Access at the NIH Regional Biocontainment Laboratory on the University of Louisville Campus Security Risk Assessment (SRA) Clearance for the US Federal Select Agent Program (FSAP)

University of Louisville LGBT Health Certificate

Indiana State Department of Education instructional license

Life Science 5-12, Chemistry 5-12, Mathematics 5-12, Physics 5-12, Physical Science 5-12

First Aid, CPR, AED, Emergency Oxygen, \& OSHA compliant blood borne pathogens instructor trainer (NAUI-DAN,ASHI)

Certified SCUBA instructor (PADI, NAUI, SDI, TDI)/Instructor trainer (NAUI)/Technical diving instructor (PADI, TDI)

\section{PROFESSIONAL MEMBERSHIPS}

American Society for Virology

American Society for Microbiology 